\title{
On finite order periodic orbits for a homeomorphism in the two-dimensional torus
}

\author{
Germán Fabian Escobar Fiesco
}

\author{
TESE APRESENTADA \\ $\mathrm{AO}$ \\ Instituto de Matemática e Estatística \\ DA \\ Universidade De SÃo Paulo \\ PARA \\ OBTENÇÃO DO TÍTULO \\ $\mathrm{DE}$ \\ DOUTOR EM CIÊNCIAS
}

Programa: Doutorado em Matemática Aplicada

Orientador: Prof. Dr. André Salles de Carvalho

Durante o desenvolvimento deste trabalho o autor recebeu auxílio financeiro da CNPq 


\section{On finite order periodic orbits for a homeomorphism in the two-dimensional torus}

Esta versão da tese contém as correções e alterações sugeridas pela Comissão Julgadora durante a defesa da versão original do trabalho, realizada em 18/06/2021. Uma cópia da versão original está disponível no Instituto de Matemática e Estatística da Universidade de São Paulo.

Comissão Julgadora:

- Prof. Dr. André Salles de Carvalho (orientador) - IME-USP (Presidente)

- Prof. Dr. Fabio Armando Tal - IME-USP

- Prof. Dr. Edson Vargas - IME-USP

- Prof. Dr. Toby Dixon Harold Hall - UL (Externo)

- Prof. Dr. Juan Valentín Mendoza Mogollón - UNIFEI (Externo) 


\section{Aknowledgements}

I would like to thank each of the people who, in different ways, helped me to complete this work. Thank you so much, to my advisor Professor André de Carvalho for his guidance and patience during my doctoral training process. I would like to thank my mother for her advice and guidance, especially my wife and daughter who, despite the distance, were my reason for continuing it.

I also thank the committee members of the pre-examination, and examination committee for reading this document. 


\section{Resumo}

ESCOBAR, FIESCO G. F. Sobre órbitas periódicas de orden finito para un homeomorfismo del toro dos dimensional. 2010. 120 f. Tese (Doutorado) - Instituto de Matemática e Estatística, Universidade de São Paulo, São Paulo, 2010.

Neste trabalho, para um específico homeomorfismo do toro isotópico à identidade, dado um vetor de rotação racional $v=\frac{k}{n}\left(p_{1}, p_{2}\right)$ no interior do conjunto de rotação, nós caracterizamos as órbitas periódicas de ordem finita com vetor de rotação $v$ e sua representação simbólica sob certas restrições.

Palavras-chave: Órbitas periódicas de ordem finita, vetores de rotação, grupo de classes de isotopia. 


\section{Abstract}

ESCOBAR, FIESCO G. F. On finite order periodic orbits for a homeomorphism in the two-dimensional torus. 2019. 50 f. Tese (Doutorado) - Instituto de Matemática e Estatística, Universidade de São Paulo, São Paulo, 2010.

In this work, for a specific torus homeomorphism isotopic to the identity, given a rational rotation vector $v=\frac{k}{n}\left(p_{1}, p_{2}\right)$ in the interior of the rotation set, we characterize under certain restrictions the finite order periodic orbits with rotation vector $v$ and their symbolic representation.

Keywords: Finite order periodic orbits, rotation vectors, isotopy mapping class group. 


\section{Contents}

1 Introduction $\quad 1$

2 Preliminaries $\quad 3$

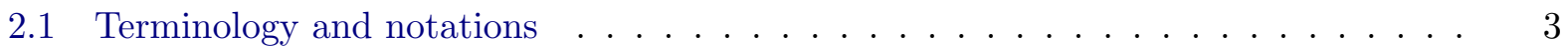

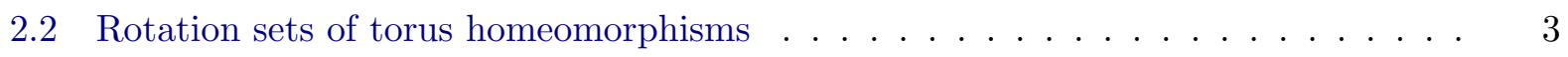

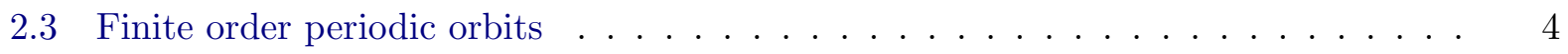

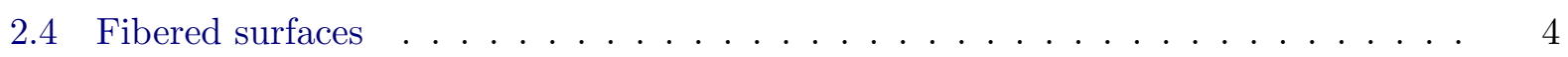

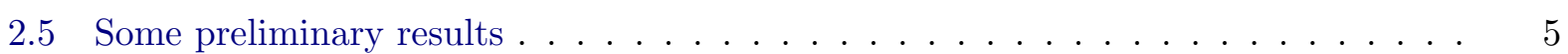

3 Description of the homeomorphism $\quad 9$

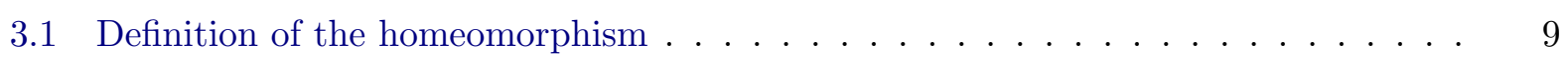

3.2 Rotation vectors for the homeomorphism . . . . . . . . . . . . . . . 12

3.3 Some preliminary results . . . . . . . . . . . . . . . . . . . 13

4 Finding finite order periodic orbits $\quad 15$



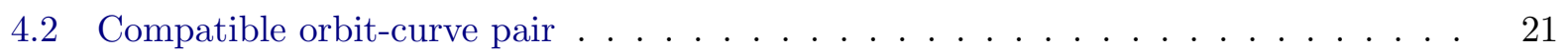

4.3 Unfoldable and unimodal orbit-curve pair . . . . . . . . . . . . . . . . . . . . . . . . . . . . . . . . . . . . .

4.4 Main theorems and proofs . . . . . . . . . . . . . . . . . 31

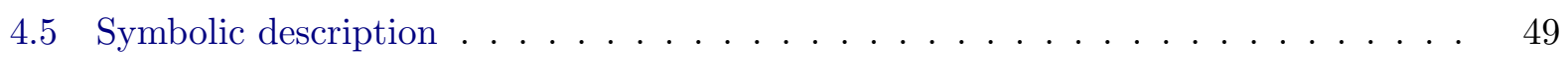

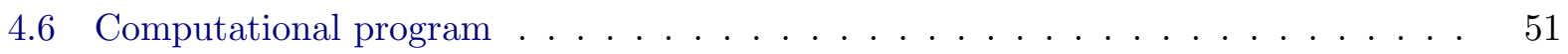

$\begin{array}{ll}\text { Bibliography } & 53\end{array}$ 


\section{Chapter 1}

\section{Introduction}

In this thesis we study the dynamics of finite order periodic orbits for a particular homeomorphism in the two-dimensional torus isotopic to identity. Using the Nielsen-Thurston classification theorem we will work with periodic orbits which have the property that the isotopy class keeping the orbit invariant as a set is of finite order.

For homeomorphisms in the annulus isotopic to identity, this orbits were called by Boyland in [Boy92] monotone periodic orbits. Now, if $f$ is a torus homeomorphism isotopic to the identity, Franks proved in [Fra89] that given a rational vector in the interior of rotation set there is a point with this rotation vector, and later Parwani showed in [Par05] that for a rational rotation vector $v=\left(\frac{p}{q}, \frac{r}{q}\right)$ the homeomorphism $f$ has a topologically monotone periodic orbit with rotation vector $v$. In other words, he proved that there is at least a periodic orbit with rotation vector $v$ which is dynamically similar to a rigid rotation induced by the translation $v$.

This work focuses on finding the largest set of finite order periodic orbits with rational rotation vector in the interior of the rotation set for the torus homeomorphism define in [BdCH16]. Although Parwani's theorem guarantees us the existence of finite order periodic orbits, there is no literature how to find them, or, how many orbits share the same rotation vector. In this proposal under certain restrictions, we present a symbolic way to find the set of finite order periodic orbits (fopos). The fopos set that we will describe corresponds to a simplification of the complete problem. We use the horizontal and vertical projections in the universal cover $\mathbb{R}^{2}$ of the torus to know the relative position of the points of a periodic orbit, we will only consider orbits with points in regions that under the action of the homeomorphism $f$, preserve their "orientation". Although it is not difficult to find fopos in the general case, since the orbits also have points in regions where $f$ reverses orientation, we can not generalize the ideas to define a common set of properties to guarantee finite order.

Our study of finite order periodic orbits is organized as follows: In Chapter 2, we present some preliminaries concepts of rotation theory, mapping class group, among others. In Section 2.4, we introduce some known results of curves in surfaces, and a classification theorem for finite order torus homeomorphism, [GLM91]. Chapter 3 contains the description of the torus homeomorphism which we are going work, and its rotation set. In particular, the Section 3.3 contains a first idea that supports the direction of our work. For a torus homeomorphism isotopic to the identity, we will see (Theorem 3.3.2) that a necessary and sufficient condition to determinate a fopo is to find a simple closed curve that contains the orbit, which is invariant up to isotopy relative to the periodic orbit. This will be our tool to represent many different fopos for a fixed rotation vector.

Finally in Chapter 4 we define the way to find finite order periodic orbits. In the Section 4.1, we describe the orbits of our work. For this, as consequence of the equivalence in 3.3, we will consider the set of periodic orbits contained in simple closed curves with the property that the orbit under the action of $f$ is "compatible" with a rotation. Since the pre-images points of any orbit are contained in a circle (we can suppose equidistant between them), we will choice periodic orbits for which the action of the homeomorphism induce a rigid rotation in the circle. This property will 
be a necessary condition, but it is not sufficient since we can find examples where a periodic orbit is compatible with a rotation, but the $f$-image of the curve that contains their points cannot be deformed continuously relative to the orbit until it return to the original curve. From this set we will find the fopos subset, that is, those orbits where the relative isotopy is possible. This raises the need to impose some type of additional condition to ensure isotopy of the curve. The Sections 4.2, 4.3 contain the mathematics that we consider necessary to guarantee the relative isotopy. In 4.4, we demonstrate what we consider our main results. The penultimate section contains the summary of the symbolic description of the periodic orbits considered. As a comment, our description also contains the fopos for rational rotation vectors $v=\left(\frac{p_{1}}{q}, \frac{p_{2}}{q}\right), q=p_{1}+2 p_{2}$ in the hypotenuse of the rotation triangle.

In the last section, we will show the design of the computational program to list the finite order periodic orbits of a given rotation vector. Our construction (Chapter 4) allow us to define an algorithm to find the number of periodic orbits and fopos associated to a rotation vector. 


\section{Chapter 2}

\section{Preliminaries}

In this chapter we present the concepts that we will use subsequent chapters. In the Section 2.1 we will give some basic notations. We present in Section 2.2 some preliminary aspects of homeomorphisms rotation theory. In the Section 2.3 we will define what a finite order periodic orbit means. In 2.4 Section we present some definitions about fibered surface. Finally in 2.5, we will present two results that will allow us to understand the construction of the next chapter.

\subsection{Terminology and notations}

A surface is a 2-dimensional manifold. By a closed curve in a surface $S$ we will mean a continuous map $\mathbb{S}^{1} \rightarrow S$. A closed curve in $S$ is simple if it is embedded, that is, if the map $\mathbb{S}^{1} \rightarrow S$ is injective. A simple closed arc will be an embedding from $[0,1] \rightarrow S$. We will denote the curves and arcs by greek letters as $\gamma, \eta, \alpha, \beta, \xi, \zeta$.

A closed topological disk $\mathbb{D}$ in a surface $S$ is the image under a topological embedding of a closed unit disk in $\mathbb{R}^{2}$. If $\operatorname{dist}(\cdot, \cdot)$ represent the distance function in $S$, for $\varepsilon>0$ small and some $p \in S$ we denote por $\mathbb{D}_{\varepsilon}=\{x \in S \mid \operatorname{dist}(x, p) \leq \varepsilon\}$.

We will write $f \simeq g($ rel. $A)$, to say that the surface homeomorphisms $f$ and $g$ are isotopic relative to the set $A$. This is, there exist a continuous map $K: S \times[0,1] \rightarrow S$ such that for all $t \in[0,1], K(x, t)$ is a homeomorphism, furthermore $K(x, 0)=f(x), K(x, 1)=g(x)$, and $K(x, t)=f(x)=g(x)$ for all $x$ in $A$. Two simple closed curves $\gamma$ and $\eta$ will be isotopic if there is a homotopy $K: \mathbb{S}^{1} \times[0,1] \rightarrow S$ such that the closed curve $K\left(\mathbb{S}^{1} \times\{t\}\right)$ is simple for each $t \in[0,1]$.

We will denote the torus homeomorphisms by letters $f, g, h$ and their lifts by $\tilde{f}, \tilde{g}, \tilde{h}$. The map projection will be written as $\pi: \mathbb{T}^{2} \rightarrow \mathbb{T}^{2} /(\sim)$, and the canonical projections in the plane $\mathbb{R}^{2}$ on the first and second factor by $\pi_{x}, \pi_{y}$.

A point $x$ is called a periodic point for a torus homeomorphism $f$, if there exist a $n \in \mathbb{N}$ such that $f^{n}(x)=x$. The least $n$ satisfying the previous equation is called the period. The set of periodic points will be denote by $\operatorname{Per}(f)$. The orbit of a periodic point $x$, denoted by $\mathcal{O}$ is the set $\left\{f^{k}(x) ; k=0,1, \ldots, n-1\right\}$.

For a simple closed curve $\gamma$ in the two-torus, we will denote its homology as $\operatorname{Hom}(\gamma)=\left(p_{1}, p_{2}\right)$ such that $p_{i} \in \mathbb{Z}, i=1,2$. It is known that the torus first homology group is given by $\mathrm{H}_{1}\left(\mathbb{T}^{2}, \mathbb{Z}\right)=$ $\mathbb{Z} \times \mathbb{Z}$.

\subsection{Rotation sets of torus homeomorphisms}

Denote by $\mathrm{Homeo}_{0}\left(\mathbb{T}^{2}\right)$ the set the homeomorphism of $\mathbb{T}^{2}$ homotopic to the identity. Suppose that $f \in$ Homeo $_{0}\left(\mathbb{T}^{2}\right)$ and $\tilde{f}: \mathbb{R}^{2} \longrightarrow \mathbb{R}^{2}$ be a lift. 
Definition 2.2.1 ([MZ89]). Let $x \in \mathbb{T}^{2}$. The pointwise rotation set is given by

$$
\rho_{p}(\tilde{f})=\bigcup_{\tilde{x} \in \mathbb{R}^{2}}\left\{\rho(\tilde{f}, \tilde{x}): \lim _{n \rightarrow \infty} \frac{\tilde{f}^{n}(\tilde{x})-\tilde{x}}{n}=\rho(\tilde{f}, \tilde{x})\right\} .
$$

The Misiurewics-Ziemian rotation set

$$
\begin{aligned}
\rho(\tilde{f}) & =\left\{\lim _{k \rightarrow \infty} \frac{\tilde{f}^{n_{k}}\left(\tilde{x}_{k}\right)-\tilde{x}_{k}}{n_{k}}: x_{k} \in \mathbb{R}^{2}, n_{k} \longrightarrow \infty\right\} \\
& =\bigcap_{n \geqslant 1} \frac{\bigcup_{m \geqslant n}\left\{\frac{\tilde{f}^{m}(\tilde{x})-\tilde{x}}{m}: \tilde{x} \in \mathbb{R}^{2}\right\}}{\bigcup_{n \geqslant 1}\left\{\frac{\tilde{f}^{m}(\tilde{x})-\tilde{x}}{m}: \tilde{x} \in[0,1]^{2}\right\}}
\end{aligned}
$$

In particular, $\rho(\tilde{f})$ is compact and convex.

Theorem 2.2.2 ([Fra89]). If $v$ is a rotation vector of rational coordinates in the interior of $\rho(\tilde{f})$, then there is a point $\tilde{x} \in \mathbb{R}^{2}$ such that $x=\pi(\tilde{x}) \in \mathbb{T}^{2}$ is a periodic point for $f$ and $v=\lim _{n \rightarrow \infty} \frac{\tilde{f}^{n}(\tilde{x})-\tilde{x}}{n}$.

\subsection{Finite order periodic orbits}

Let $S$ be a surface. We will denote by $S_{g, n}$ a surface of genus $g$ with $n$ punctures and empty boundary, and by Homeo ${ }^{+}(S)$ the group of orientation-preserving homeomorphism of $S$.

Definition 2.3.1. Let $S$ be a surface. Define the mapping class group of $S$, denoted $\operatorname{Mod}(S)$ as follow

$$
\operatorname{Mod}(S)=\pi_{0}\left(\operatorname{Homeo}^{+}(S)\right) .
$$

Theorem 2.3.2 (Nielsen-Thurston classification). Let $g, n \geqslant 0$. Each $f \in \operatorname{Mod}\left(S_{g, n}\right)$ is either periodic, reducible, or pseudo-Anosov.

Given a periodic orbit, we will introduce them as marked points in the surface and use the Nilsen-Thurston classification theorem to study the isotopy class relative to the periodic orbit.

Definition 2.3.3 (Finite order periodic orbit). It will be said that a $f$-periodic orbit is of finite order (or fopo) if the isotopy class of $f$, relative to the periodic orbit is of finite order.

\section{$2.4 \quad$ Fibered surfaces}

In this section we introduce some definitions about fibered surface (see [FM93, BH95, dCH01]). Fibered surface is understood as compact surface $S$ which can be decomposed in arcs on strips or topological disk. In particular, a fibered surface $S$ contains a compact subsurface obtained from a graph $X$ in which each point has been thickened up, either to a disk or an arc. More precisely,

Definition 2.4.1. A thick graph is a pair $(S, \mathbb{X})$, where $S$ is a closed orientable surface with a fixed metric compatible with its topology and $\mathbb{X}$

1. Each decomposition element of $\mathbb{X}$ is either a leaf homeomorphic to $[0,1]$, or a junction homeomorphic to $\mathbb{D}$ (the unit disk in $\mathbb{R}^{2}$ ).

2. The boundary in $\mathbb{X}$ of each junction is a finite number of disjoint arcs: if there are $k$ such arcs, then the disk is called a $k$-junction.

3. The set of $k$-junctions with $k \neq 2$ is finite. 
4. Each decomposition element which is not in the accumulation of a sequence of distinct 2junctions is contained in a chart as depicted in Figure 2.1.

5. Each component of $S \backslash \mathbb{X}$ is an open disk.

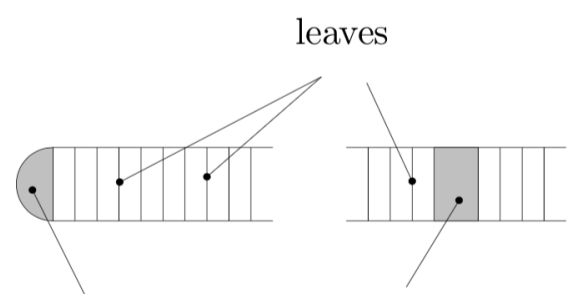

1-junction

2-junction

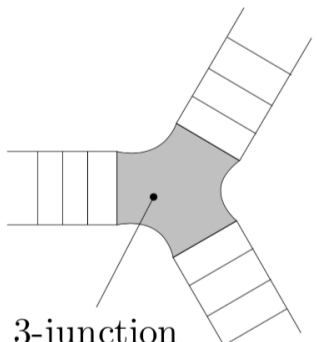

3-junction

Figure 2.1: Charts in a thick graph

If $(S, \mathbb{X})$ is a thick graph, let $\sim$ be the equivalence relation on $\mathbb{X}$ given by $x \sim y$ if and only if $x$ and $y$ lie in the same decomposition element. Then $X=\mathbb{X} /(\sim)$ is a graph, whose vertices correspond to the junctions of $\mathbb{X}$. The vertex set of $X$ will be denoted $V$, and the union of the junctions of $\mathbb{X}$ will be denoted $\mathbb{V}$ : thus $\mathbb{V}=\pi^{-1}(V)$. The components of $\mathbb{X} \backslash \mathbb{V}$ are called strips: each strip is therefore homeomorphic to $(0,1) \times[0,1]$.

If $(S, \mathbb{X})$ is a thick graph and $f:(S, \mathbb{X}) \longrightarrow(S, \mathbb{X})$ is a homeomorphism (i.e., a homeomorphism $f: S \longrightarrow S$ with $f(\mathbb{X}) \subset \mathbb{X})$ under which the image of each decomposition element of $\mathbb{X}$ is contained in a decomposition element, then $\left.f\right|_{\mathbb{X}}$ induces a graph endomorphism $F: X \longrightarrow X$ such that $\left.\pi \circ f\right|_{\mathbb{X}}=F \circ \pi$.

Definition 2.4.2. A thick graph map of $(S, \mathbb{X})$ is an orientation-preserving homeomorphism $f:(S, \mathbb{X}) \longrightarrow(S, \mathbb{X})$ such that:

1. $f(\mathbb{X}) \subset \operatorname{Int}(\mathbb{X})$.

2. If $\gamma$ is a decomposition element of $\mathbb{X}$, then $f(\gamma)$ is contained in a decomposition element, and $\operatorname{diam}\left(f^{n}(\gamma)\right) \rightarrow 0$ as $n \rightarrow \infty$.

3. The induced graph endomorphism $F: X \longrightarrow X$ is piecewise monotone (that is, there is a finite subset $L$ of $V$ such that $F^{-1}(x) \cap U$ is connected for each $x \in X$ and each component $U$ of $X \backslash L)$; and is strictly monotone away from the preimages of vertices (that is, every $x \in X \backslash F^{-1}(V)$ has a neighborhood on which $F$ restricts to an embedding).

4. For each component $U$ of $S \backslash \mathbb{X}$ there is a (least) positive integer $n_{U}$ for which either $f^{n_{U}}(U) \subset$ $\mathbb{X}$ or $f^{n_{U}}(U) \cap U \neq \emptyset$, in which case $U$ contains a period $n_{U}$ point $p_{U}$ of $f$, which is a source whose immediate basin contains $U$ : that is, $f^{-k n_{U}}(x) \rightarrow p_{U}$ as $k \rightarrow \infty$ for all $x \in U$.

\subsection{Some preliminary results}

Now, we list some classical results which will be used in what follows. Goods references are the books [Bus10], [FM12], [Why58], [MT01], [Lee11].

We will denote the 2-dimensional plane by $\mathbb{R}^{2}$. A Jordan curve $J$ is a simple closed curve.

Theorem 2.5.1 (Jordan-Schoenflies). Every simple closed curve $J$ divides the plane into exactly two components of each of which it is the complete boundary and the closure of the bounded component (interior domain) can be mapped topologically onto the closed unit disc. 
Corollary 2.5.2. Let $p, q \in \mathbb{D}$ (possibly with $p=q$ ) and let $\alpha$ and $\beta$ be simple arcs connecting $p$ to $q$ which, except possibly for the endpoints, are contained in the interior of $\mathbb{D}$. Then there exists a homeomorphism $h: \mathbb{D} \longrightarrow \mathbb{D}$ isotopic to identity with $\{p, q\} \cup \partial S$ point-wise fixed such that $h \circ \alpha=\beta$ or $h \circ \alpha=\beta^{-1}$.

Definition 2.5.3. Let $J^{\circ}$ be an interior domain in the plane, a cross-cut in $J^{\circ} \cup J$ is a simple closed arc $\alpha$ such that $\alpha^{\circ} \subset J^{\circ}$ and $\partial \alpha \subset J$.

Theorem 2.5.4. Let $J$ be a simple close curve in the plane. If $\alpha$ is a cross-cut in $J^{\circ}$, then $J^{\circ}$ is divide in exactly two domain with frontiers $\alpha \cup J_{1}, \alpha \cup J_{2}$, for $J_{1} \cup J_{2}=J \backslash \partial \alpha$.

Corollary 2.5.5 ([Kol94]). Let $\alpha_{0} \cup \alpha_{1} \cup \cdots \cup \alpha_{n}(n \geq 1)$ be simple arcs. If $\alpha_{0} \cup \alpha_{1}$ is a simple closed curve and $\alpha_{2} \cup \cdots \cup \alpha_{n}$ is a collection of cross-cuts in $\left(\alpha_{0} \cup \alpha_{1}\right)^{\circ}$, then the set $\mathbb{R}^{2} \backslash\left(\alpha_{0} \cup \alpha_{1} \cup \cdots \cup \alpha_{n}\right)$ has exactly $n$ distinct bounded components, each of them is a topological disk whose boundary lies in $\alpha_{0} \cup \alpha_{1} \cup \cdots \cup \alpha_{n}$.

Theorem 2.5.6 ([CK94]). Let $\mathbb{D}_{1}, \ldots, \mathbb{D}_{n}$ be a finite number of closed topological disks in the plane and $J^{\circ}$ be any connected component of $\cap_{i=1}^{n} \mathbb{D}_{i}^{\circ}$. Then $\partial J$ is a simple closed curve and $J$, the closure of $J^{\circ}$ is a topological disc.

Let $f, g: I \rightarrow Y$ be paths. We say that $f$ and $g$ are composable paths if $f(1)=g(0)$.

Definition 2.5.7. If $f$ and $g$ are composable paths, we define their product $f \cdot g$ by

$$
f \cdot g(s)= \begin{cases}f(2 s) & 0 \leq s \leq \frac{1}{2} \\ g(2 s-1) & \frac{1}{2} \leq s \leq 1 .\end{cases}
$$

Theorem 2.5.8. Let $f_{0} \simeq f_{1}$ and $g_{0} \simeq g_{1}$ be homotopy paths. If $f_{0}$ and $g_{0}$ are composable, then $f_{1}$ and $g_{1}$ are composable and $f_{0} \cdot g_{0} \simeq f_{1} \cdot g_{1}$.

Theorem 2.5.9 (Path Lifting Property). Let $p: X \rightarrow Y$ be a covering map. Suppose $f: I \rightarrow Y$ is any path, and $x_{0} \in X$ is any point in the fiber of $p$ such that $p\left(x_{0}\right)=f(0)$. Then there exists a unique lift $\tilde{f}: I \rightarrow X$ of $f$ such that $p \circ \tilde{f}=f$ and $\tilde{f}(0)=x_{0}$.

Theorem 2.5.10 (Monodromy Theorem). Let $p: X \rightarrow Y$ be a covering map. Suppose $f$ and $g$ are paths in $Y$ with the same initial point and the same end point, and $\tilde{f}_{x_{0}}, \tilde{g}_{x_{0}}$ are their lifts with the same initial point $x_{o} \in X$.

(i) $\tilde{f}_{x_{0}} \simeq \tilde{g}_{x_{0}}$ if and only if $f \simeq g$.

(ii) If $f \simeq g$, then $\tilde{f}_{x_{0}}(1)=\tilde{g}_{x_{0}}(1)$.

(" $\simeq "$ represent homotopy).

Theorem 2.5.11. Let $S$ be a surface. If $H: \mathbb{S}^{1} \times[0,1] \longrightarrow S$ is a smooth isotopy of simple closed curves, then there is an isotopy $K: S \times[0,1] \longrightarrow S$ so that $\left.K\right|_{S \times 0}$ is the identity and $\left.K\right|_{H\left(\mathbb{S}^{1} \times 0\right) \times[0,1]}=H$.

Let $\Gamma_{1}$ be the group generated by the translations $(1,0),(0,1)$, and let $\Gamma_{2}$ the group generated by the translations $(1,0),\left(\frac{1}{2}, \frac{\sqrt{3}}{2}\right)$. Let $\mathbb{T}^{2}=\mathbb{R}^{2} / \Gamma_{i}, i=1,2$. Let $\bar{T}_{x}$ be a translation by $x$ on $\mathbb{R}^{2}$ this is, $\bar{T}_{x}(y)=x+y$, for $x, y \in \mathbb{R}^{2}$. Let $\bar{R}_{w}, w \in \mathbb{R} / 2 \pi \mathbb{Z}$ be a rotation about 0 on $\mathbb{R}^{2}$ by angle $w$. And let $\bar{r}$ be the reflection on the $x$ axis at $\mathbb{R}^{2}$. Denote by $T_{x}, R_{w}, r$ the maps on $\mathbb{T}^{2}$ so that, $\pi \circ \bar{T}_{x}=T_{x} \circ \pi, \pi \circ \bar{R}_{w}=R_{w} \circ \pi, \pi \circ \bar{r}=r \circ \pi$.

Theorem 2.5.12 ([GLM91]). If $f: \mathbb{T}^{2} \longrightarrow \mathbb{T}^{2}$ is a homeomorphism of finite order, then it is orientation-preserving conjugate to one of the following:

(i) $T_{m / n}: \mathbb{R}^{2} / \Gamma_{1} \rightarrow \mathbb{R}^{2} / \Gamma_{1}$; for $m \in \mathbb{Z}^{2}, n \in \mathbb{N}$ (order $n$ ). 
(ii) $R_{w}: \mathbb{R}^{2} / \Gamma_{1} \rightarrow \mathbb{R}^{2} / \Gamma_{1}$; for $w= \pm \pi / 2$ or $R_{w^{\prime}}: \mathbb{R}^{2} / \Gamma_{2} \rightarrow \mathbb{R}^{2} / \Gamma_{1} ; w^{\prime}= \pm \pi / 3, \pm 2 \pi / 3$ (orders 4,2,6,3 respectively).

(iii) $r \circ T_{m / n}: \mathbb{R}^{2} / \Gamma_{1} \rightarrow \mathbb{R}^{2} / \Gamma_{1}$; for $m \in \mathbb{Z}^{2}, n \in \mathbb{N}$ (order $m$; if $m$ is even, and order $2 m$; if $m$ is odd).

(iv) $r \circ R_{\pi / 2}: \mathbb{R}^{2} / \Gamma_{1} \rightarrow \mathbb{R}^{2} / \Gamma_{1}$ (order 2).

REMARK. Note that $m / n$ for $(i)$ and $(i i i)$ is not unique, many of these are orientation-preserving conjugate. 


\section{Chapter 3}

\section{Description of the homeomorphism}

In [BdCH16] was introduce on the figure eight space $X=S_{1} \wedge S_{2}$ a map $F: X \longrightarrow X$ homotopic to the identity. From this map, in the first section we will describe on the two-torus a homeormorphism isotopic to the identity which contain the fibered surface $\mathbb{X}$, such that $X=\mathbb{X} /(\sim)$. Followed by this, we will define an order on the periodic orbit points, which is naturally induced by fibered surface. In Section 3.2, we will identify the rotation set associated to the homeomorphism defined in 3.1. Finally in 3.3, we will give some affirmations and their respective proofs. We will prove for torus homeomorphisms isotopic to identity, that a necessary and sufficient condition to show that a periodic orbit with rotation vector $v=\frac{k}{n}\left(p_{1}, p_{2}\right)$ is of finite order, is that, there exist a simple closed curve containing the orbit with homology $\left(p_{1}, p_{2}\right)$ invariant up to isotopy relative to the orbit. This tool allow us to find different fopos for a fixed the rotation vector.

\subsection{Definition of the homeomorphism}

Let $X=S_{1} \wedge S_{2}$ be a wedge of two oriented circles, with respective lengths 5 and 3 , which will be denoted by $a_{1}, \ldots, a_{5}, b_{1}, \ldots, b_{3}$ respectively, such that $S_{1}=a_{1} a_{2} a_{3} a_{4} a_{5}$ and $S_{2}=b_{1} b_{2} b_{3}$. Let $F: X \longrightarrow X$ be the homeomorphism homotopic to the identity, which expands each edge uniformly by a factor of either 5 or 3 (see figure 3.1).

$$
\begin{aligned}
& S_{1}: \quad F\left(a_{1}\right)=S_{2} \quad F\left(a_{2}\right)=S_{2}^{-1} \quad F\left(a_{3}\right)=S_{1} \quad F\left(a_{4}\right)=S_{1} \quad F\left(a_{5}\right)=S_{1}^{-1} \\
& S_{2}: \quad F\left(b_{1}\right)=S_{2} \quad F\left(b_{2}\right)=S_{1} \quad F\left(b_{3}\right)=S_{2}^{-1}
\end{aligned}
$$

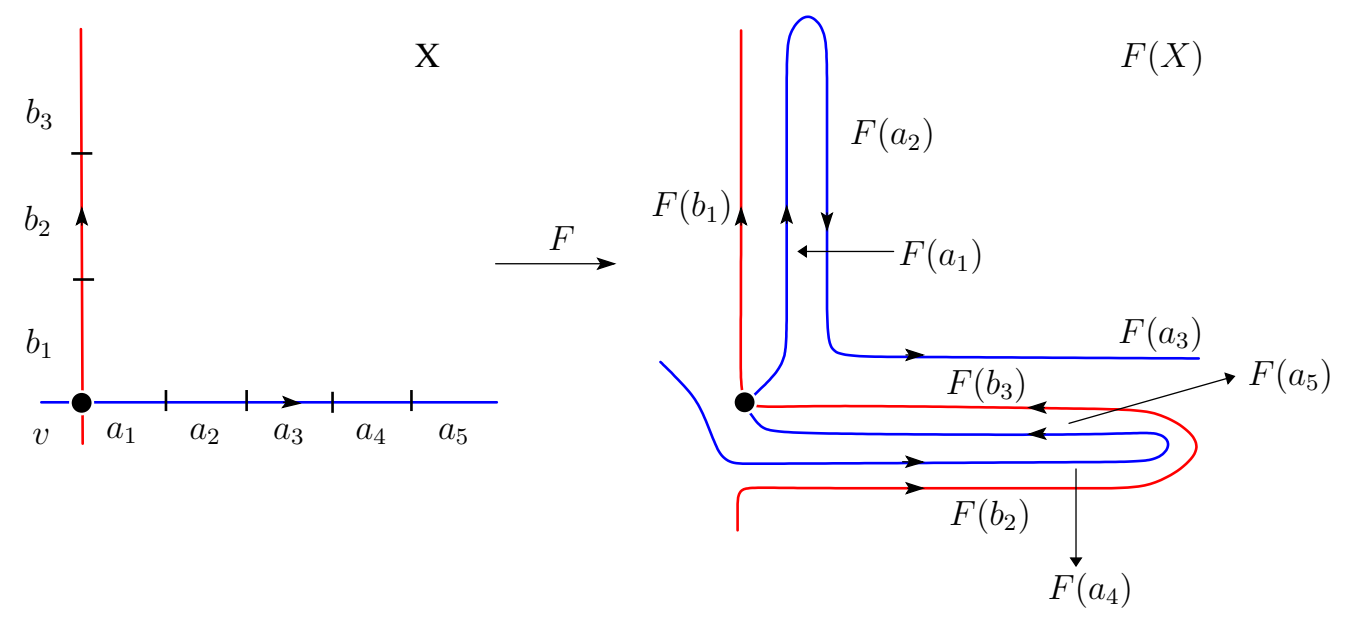

Figure 3.1: Action of the homeomorphism $F$ on the set $X=S_{1} \wedge S_{2}$

Definition 3.1.1. Let $\left(\mathbb{T}^{2}, \mathbb{X}\right)$ be a thick graph, where $\mathbb{X}$ is a fibered subsurface modelled by a $4^{-}$ junction $\mathbb{V}$, and two fibered strips $\boldsymbol{a}, \boldsymbol{b}$ which looks like the Figure 3.2 (Note that $X=\mathbb{X} /(\sim)$ is a 
graph embedded in $\mathbb{X}$, with a vertice $v=\mathbb{V} /(\sim)$ of valence four $)$.

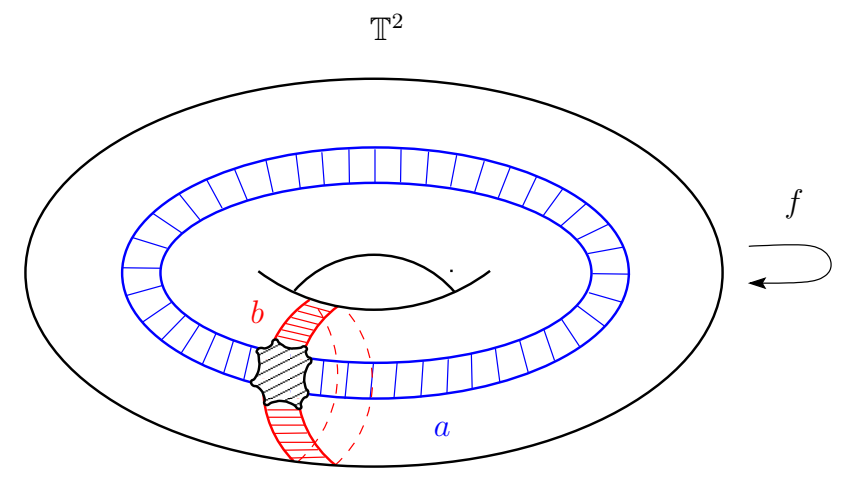

Figure 3.2: Representation in the two-dimensional torus of the $\boldsymbol{a}, \boldsymbol{b}$ strips.

Let us define the homeomorphism $f:\left(\mathbb{T}^{2}, \mathbb{X}\right) \longrightarrow\left(\mathbb{T}^{2}, \mathbb{X}\right)$ (thick graph map) described in Figure 3.3, such that $f$ induces the graph endomorphism $F: X \longrightarrow X$, with $\left.\pi \circ f\right|_{\mathbb{X}}=F \circ \pi, \pi: \mathbb{X} \longrightarrow X$.

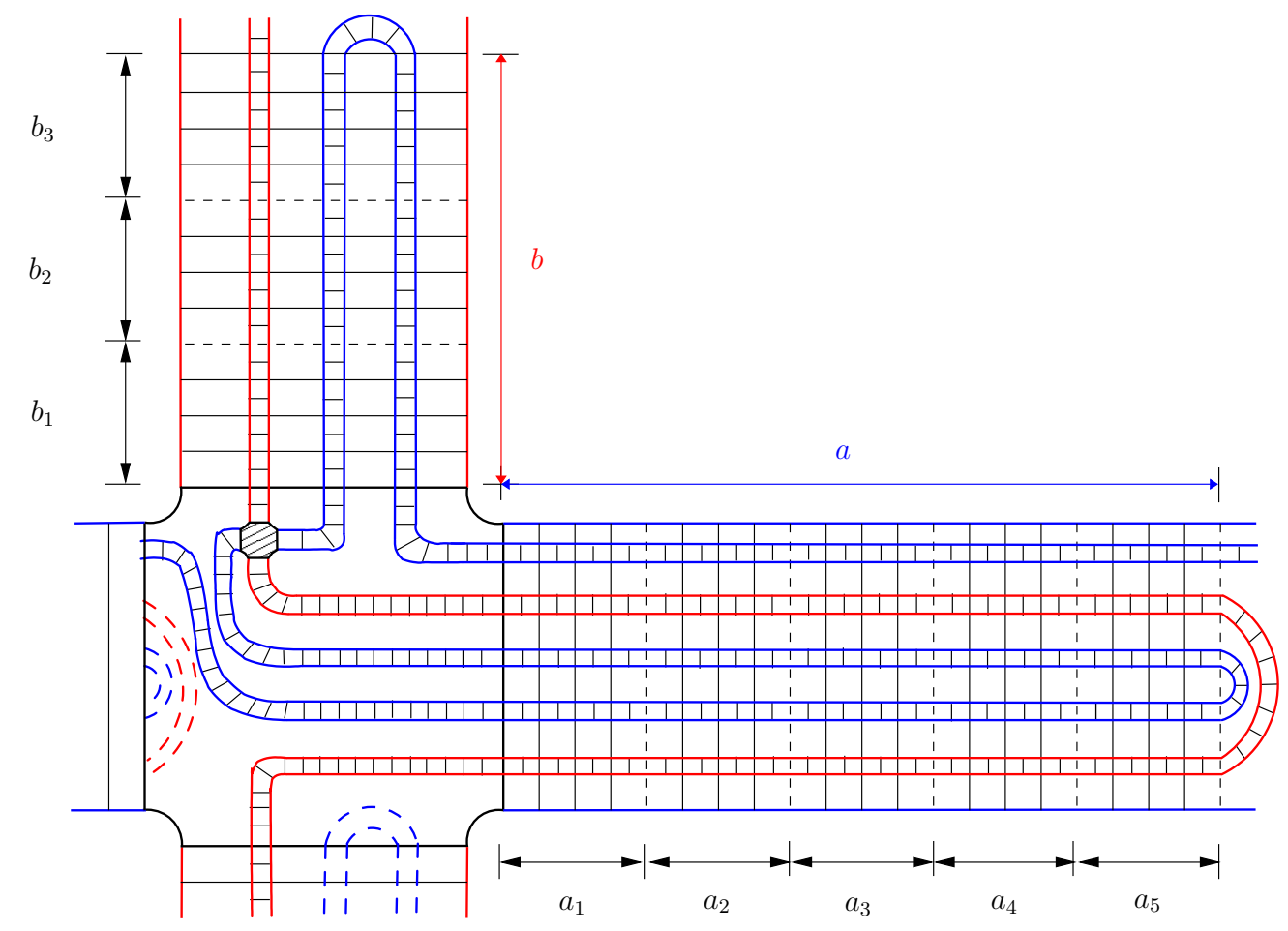

Figure 3.3: $f$-image of the $\boldsymbol{a}, \boldsymbol{b}$ strips.

Notation. From now on, we denote the regions on the $\boldsymbol{a}, \boldsymbol{b}$ strips with bold letter. The points in these regions will be written normally.

Convention. Since we are considering a fibered surface which orbit points are localized in different leafs of the subsurface $\mathbb{X}$ in a fundamental domain $D$ in the universal cover $\mathbb{R}^{2}$, we can use the projections onto the first and second factor $\pi_{x}, \pi_{y}$ to define a order on the points in the horizontal and vertical strips, as follows

$$
\left\{\begin{array}{l}
\pi_{x}\left(\tilde{a}_{1}\right)<\pi_{x}\left(\tilde{a}_{2}\right)<\pi_{x}\left(\tilde{a}_{3}\right)<\pi_{x}\left(\tilde{a}_{4}\right)<\pi_{x}\left(\tilde{a}_{5}\right) ; \\
\pi_{y}\left(\tilde{b}_{1}\right)<\pi_{y}\left(\tilde{b}_{2}\right)<\pi_{y}\left(\tilde{b}_{3}\right) .
\end{array}\right.
$$

These orders will allow us to define the relative positions (natural number) of the points of a 
periodic orbit from their symbolic representations. For example, comparing $a_{1} b_{1}, a_{1} b_{2}$, we see that the first $a_{1}$ is to the left of the second, and $b_{1} b_{2} a_{3}$ is smaller in the order above than $b_{1} b_{2} a_{4}$.
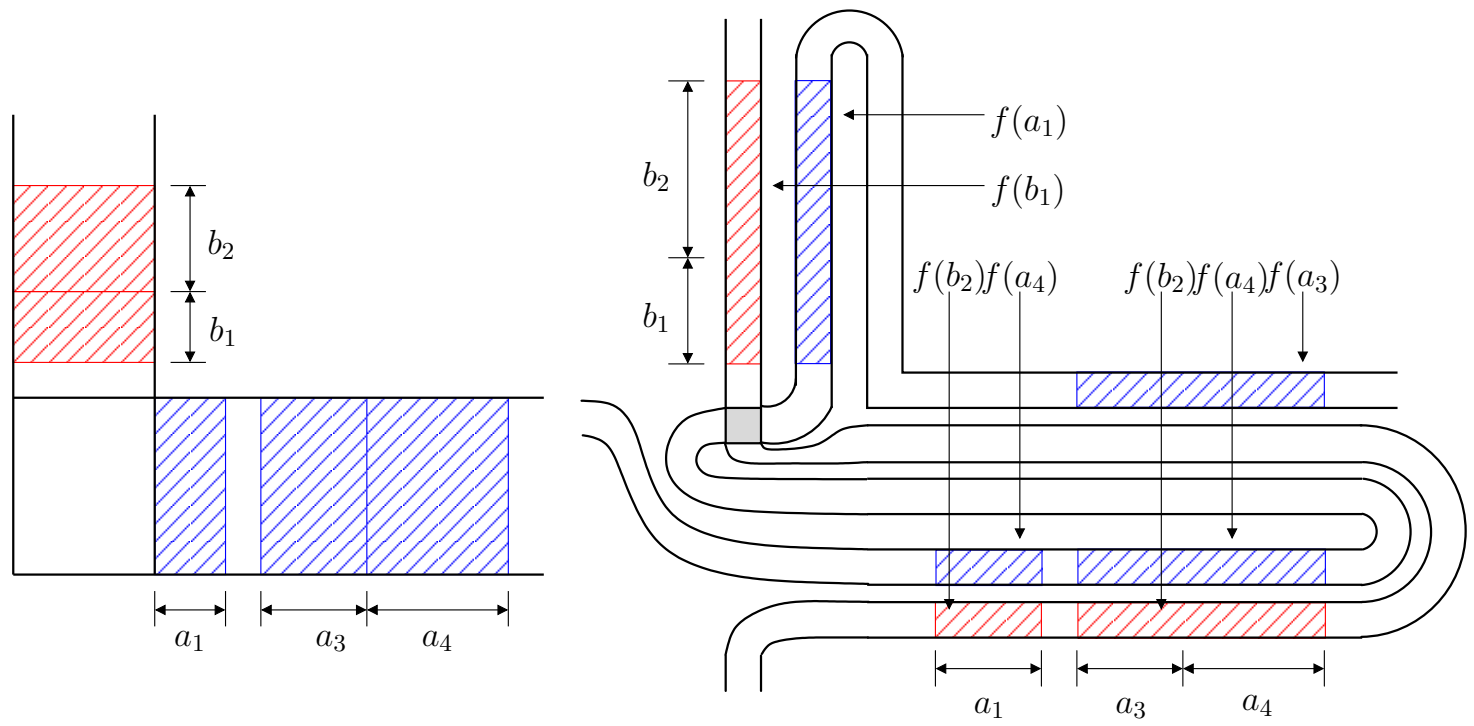

Figure 3.4

For our work, we will take advantage of the fact that $f$ preserves the previous order on the regions $\boldsymbol{a}_{1}, \boldsymbol{a}_{3}, \boldsymbol{a}_{\mathbf{4}}, \boldsymbol{b}_{\mathbf{1}}$, and $\boldsymbol{b}_{\mathbf{2}}$. For this reason, we will focus our study on periodic orbits that have points in these places (see figure 3.4). Note also that these regions satisfy the relations in the equation (3.2). Now, if $\tilde{f}$ is a lift of $f$ to the universal cover, $\mathbb{R}^{2}$, the lift of points of a periodic orbit satisfy the relations in the equation (3.2). From Definition 3.1.1, $\tilde{f}$ translate horizontally the lift points in $\boldsymbol{a}_{\mathbf{4}}$ from a fundamental domain to another by $(1,0)$ (i.e, $\tilde{a}_{4} \in \tilde{\boldsymbol{a}}, \tilde{f}\left(\tilde{a}_{4}\right) \in \tilde{\boldsymbol{a}}+(1,0)$ ), and translate vertically by $(0,1)$ the lift the points in $\tilde{\boldsymbol{b}}_{\mathbf{2}}$ (i.e, $\bar{b}_{2} \in \tilde{\boldsymbol{b}}, \tilde{f}\left(\tilde{b}_{2}\right) \in \tilde{\boldsymbol{b}}+(0,1)$ ). The points in the sectors $\boldsymbol{a}_{\mathbf{1}}, \boldsymbol{a}_{\mathbf{3}}$, and $\boldsymbol{b}_{\mathbf{1}}$ do not change of fundamental domain under the action of $\tilde{f}$.

$$
\begin{aligned}
& a_{1} \longmapsto f\left(a_{1}\right) \subset \boldsymbol{b} \quad \tilde{a}_{1} \longmapsto \tilde{f}\left(\tilde{a}_{1}\right) \subset \tilde{\boldsymbol{b}}+(0,0) \\
& a_{3} \longmapsto f\left(a_{3}\right) \subset \boldsymbol{a} \quad \tilde{a}_{3} \longmapsto \tilde{f}\left(\tilde{a}_{3}\right) \subset \tilde{\boldsymbol{a}}+(0,0) \\
& a_{4} \longmapsto f\left(a_{4}\right) \subset \boldsymbol{a} \quad \tilde{a}_{4} \longmapsto \tilde{f}\left(\tilde{a}_{4}\right) \subset \tilde{\boldsymbol{a}}+(1,0) \\
& b_{1} \longmapsto f\left(b_{1}\right) \subset \boldsymbol{b} \quad \tilde{b}_{1} \longmapsto \tilde{f}\left(\tilde{b}_{1}\right) \subset \tilde{\boldsymbol{b}}+(0,0) \\
& b_{2} \longmapsto f\left(b_{2}\right) \subset \boldsymbol{a} \quad \tilde{b}_{2} \longmapsto \tilde{f}\left(\tilde{b}_{2}\right) \subset \tilde{\boldsymbol{a}}+(0,1)
\end{aligned}
$$

In this way, we know the relative positions of any pair of points in the periodic orbit.

$$
\left\{\begin{array}{l}
\pi_{x}\left(a_{1}\right)<\pi_{x}\left(a_{1}^{\prime}\right) \quad \Longrightarrow \quad \pi_{y}\left(f\left(a_{1}\right)\right)<\pi_{y}\left(f\left(a_{1}^{\prime}\right)\right) \\
\pi_{x}\left(a_{3}\right)<\pi_{x}\left(a_{3}^{\prime}\right) \quad \Longrightarrow \pi_{x}\left(f\left(a_{3}\right)\right)<\pi_{x}\left(f\left(a_{3}^{\prime}\right)\right) \\
\pi_{x}\left(a_{4}\right)<\pi_{x}\left(a_{4}^{\prime}\right) \quad \Longrightarrow \pi_{x}\left(f\left(a_{4}\right)\right)<\pi_{x}\left(f\left(a_{4}^{\prime}\right)\right) \\
\pi_{y}\left(b_{1}\right)<\pi_{y}\left(b_{1}^{\prime}\right) \quad \Longrightarrow \pi_{y}\left(f\left(b_{1}\right)\right)<\pi_{y}\left(f\left(b_{1}^{\prime}\right)\right) \\
\pi_{y}\left(b_{2}\right)<\pi_{y}\left(b_{2}^{\prime}\right) \quad \Longrightarrow \pi_{x}\left(f\left(b_{2}\right)\right)<\pi_{x}\left(f\left(b_{2}^{\prime}\right)\right)
\end{array}\right.
$$

To end this section, we describe some differences between the cases $p_{1}>p_{2}$ and $p_{2}>p_{1}$ for a periodic orbit of rotation vector $v=\frac{k}{n}\left(p_{1}, p_{2}\right)$ : 


\begin{tabular}{|c|c|c|}
\hline$p_{1}>p_{2}$ & & $p_{2}>p_{1}$ \\
\hline$a_{4} \longmapsto f\left(a_{4}\right)=\{$ & $\left\{\begin{array}{l}a_{1} \\
a_{3} \\
a_{4}\end{array}\right.$ & $a_{4} \longmapsto f\left(a_{4}\right)=\left\{a_{1}\right.$ \\
\hline$b_{2} \longmapsto f\left(b_{2}\right)=\{$ & $\left\{\begin{array}{l}a_{3} \\
a_{4}\end{array}\right.$ & $b_{2} \longmapsto f\left(b_{2}\right)=\left\{\begin{array}{l}a_{1}, \\
a_{3}, \\
a_{4}\end{array}\right.$ \\
\hline \multicolumn{3}{|c|}{$a_{1} \longmapsto f\left(a_{1}\right)=\left\{\begin{array}{l}b_{1}, \\
b_{2}\end{array}\right.$} \\
\hline \multicolumn{3}{|c|}{$a_{3} \longmapsto f\left(a_{3}\right)=\left\{\begin{array}{l}a_{3}, \\
a_{4}\end{array}\right.$} \\
\hline \multicolumn{3}{|c|}{$b_{1} \longmapsto f\left(b_{1}\right)=\left\{\begin{array}{l}b_{1}, \\
b_{2}\end{array}\right.$} \\
\hline
\end{tabular}

Table 3.1: Combinatory of the $f$-images of periodic orbit with points in $\boldsymbol{a}_{\mathbf{1}}, \boldsymbol{a}_{\mathbf{3}}, \boldsymbol{a}_{\mathbf{4}}, \boldsymbol{b}_{\mathbf{1}}, \boldsymbol{b}_{\mathbf{2}}$.

\subsection{Rotation vectors for the homeomorphism}

From Definition 3.1.1, we can describe the rotation set as the convex hull of the rotation vectors of periodic orbits of minimal loops. If the symbols $\ell, r$ represent the vertical and horizontal Deck transformations in the transition matrix down, the minimal loops are given by the vectors $(0,0)$, $(0,1 / 2)$, and $(1,0)$. This vectors define a polygon $\Delta$ of rational vertices $(0,0),(0,1 / 2)$, and $(1,0)$. The torus homeomorphism $f$ is isotopic to the identity with rotation set of interior not empty, from Parwani [Par05] every rotation vector of the way $\left(\frac{p}{q}, \frac{q}{r}\right)$ in the interior of $\Delta$ is realized by at least a finite order periodic orbit. Let $k, n, p_{1}, p_{2}$ natural numbers with $p_{1}, p_{2}$ relatively prime, and $k / n$ irreducible. The rational rotation vectors in the interior of the rotation set can be represented as $v=\frac{k}{n}\left(p_{1}, p_{2}\right)$ with $n>k\left(p_{1}+2 p_{2}\right)$.

\begin{tabular}{|c|c|c|c|c|c|c|c|}
\hline$a_{1}$ & $a_{2}$ & $a_{3}$ & $a_{4}$ & $a_{5}$ & $b_{1}$ & $b_{2}$ & $b_{3}$ \\
\hline$a_{1} / 0$ & 0 & 0 & 0 & 0 & 1 & 1 & 1 \\
\hline 0 & 0 & 0 & 0 & 0 & 1 & 1 & 1 \\
\hline 1 & 1 & 1 & 1 & 1 & 0 & 0 & 0 \\
\hline$r$ & $r$ & $r$ & $r$ & $r$ & 0 & 0 & 0 \\
\hline$r$ & $r$ & $r$ & $r$ & $r$ & 0 & 0 & 0 \\
\hline 0 & 0 & 0 & 0 & 0 & 1 & 1 & 1 \\
\hline$\ell$ & $\ell$ & $\ell$ & $\ell$ & $\ell$ & 0 & 0 & 0 \\
\hline$\ell$ & $\ell$ & $\ell$ & $\ell$ & $\ell$ & 0 & 0 & 0 \\
\hline
\end{tabular}

The sequences of points in a periodic orbit that take points in the regions $\boldsymbol{a}_{\mathbf{1}}, \boldsymbol{a}_{\mathbf{3}}, \boldsymbol{a}_{\mathbf{4}}, \boldsymbol{b}_{\mathbf{1}}, \boldsymbol{b}_{\mathbf{2}}$ can be represented across of the transition diagram down. 

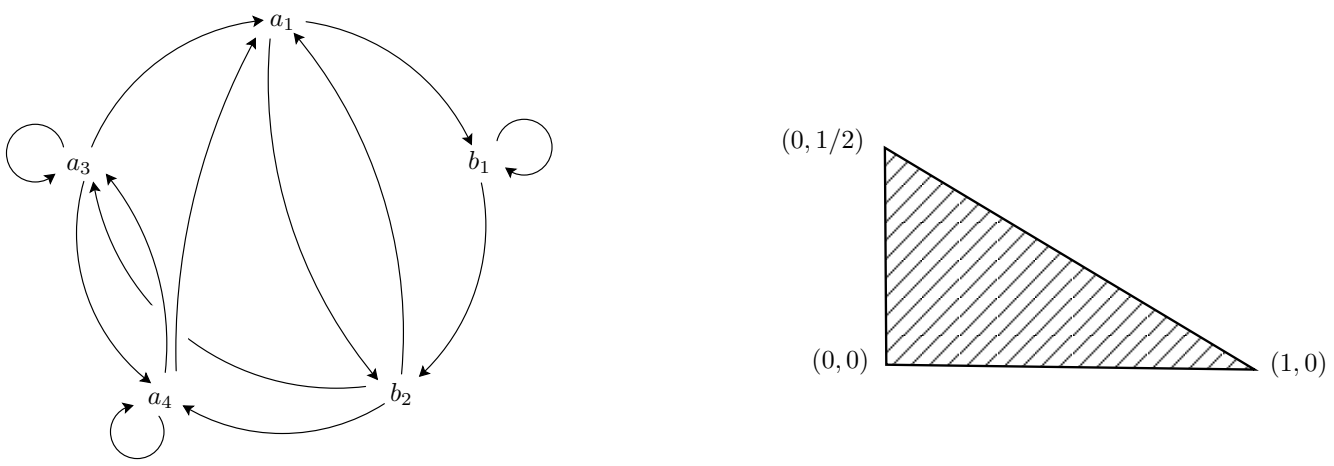

Figure 3.5: (Left.) Transition diagram to the periodic orbits with points in the regions: $\boldsymbol{a}_{\mathbf{1}}, \boldsymbol{a}_{\mathbf{3}}, \boldsymbol{a}_{\mathbf{4}}, \boldsymbol{b}_{\mathbf{1}}, \boldsymbol{b}_{\mathbf{2}}$. (Right.) Rotation set for the homeomorphism $f$.

In particular, when $n=k\left(p_{1}+2 p_{2}\right)$ the rotation vector $v$ belong to the hypothenuse of rotation triangle $\Delta$ (i.e., for $v=\frac{1}{q}\left(p_{1}, p_{2}\right)$, with $\left.q=p_{1}+2 p_{2}\right)$. This vectors can be to realized by periodic orbits with terms in the regions $\boldsymbol{a}_{\mathbf{1}}, \boldsymbol{a}_{\mathbf{4}}$, and $\boldsymbol{b}_{\mathbf{2}}$.

\subsection{Some preliminary results}

Let $m=\left(m_{1}, m_{2}\right) \in \mathbb{Z}^{2}, n \in \mathbb{N}$ such that $m_{1}, m_{2}, n$ are relatively prime. Let $T_{m / n}: \mathbb{T}^{2} \longrightarrow \mathbb{T}^{2}$ the homeomorphism induced by translation $\bar{T}_{m / n}: \mathbb{R}^{2} \longrightarrow \mathbb{R}^{2}$, such that $\pi \circ \bar{T}_{v}=T_{v} \circ \pi$.

Proposition 3.3.1. If $g: \mathbb{T}^{2} \longrightarrow \mathbb{T}^{2}$ is a torus homeomorphism isotopic to the identity with $g^{n}=i d_{\mathbb{T}^{2}}$, then $g$ is topologically conjugate to $T_{m / n}$.

Proof. Suppose that $g$ is isotopic to the identity, it follows that every lift $G$ commute with the integer translations, i.e., $G(x+v)=G(x)+v$ for all $x \in \mathbb{R}^{2}$ and $v \in \mathbb{Z}^{2}$. By Theorem 2.5.12, $g$ is conjugated to a homeomorphism of the types (i) to (iv). In particular, $g$ is not conjugated to (ii), (iii), (iv). Let us see (ii), the others cases are similar.

Assume that $g$ is conjugated to (ii). Let $g=h \circ R_{w} \circ h^{-1}$, it follows that $G=H \circ \bar{R}_{w} \circ H^{-1}$ is a lift of $g$, where $H, H^{-1}$ are lifts of $h, h^{-1}$ respectively. From $G(x+v)=G(x)+v$, we see that

$$
\begin{aligned}
\pi \circ G(x+v) & =\pi\left(H\left(\bar{R}_{w}\left(H^{-1}(x+v)\right)\right)\right) \\
& =h\left(\pi\left(\bar{R}_{w}\left(H^{-1}(x+v)\right)\right)\right) \\
& =h\left(R_{w}\left(\pi\left(H^{-1}(x+v)\right)\right)\right), \\
\pi \circ(G(x)+v) & =\pi\left(H\left(\bar{R}_{w}\left(H^{-1}(x)\right)\right)\right) \\
& =h\left(\pi\left(\bar{R}_{w}\left(H^{-1}(x)\right)\right)\right) \\
& =h\left(R_{w}\left(\pi\left(H^{-1}(x)+v\right)\right)\right) \\
& =h\left(\pi\left(\bar{R}_{w}\left(H^{-1}(x)+v\right)\right)\right) .
\end{aligned}
$$

From (3.4) and (3.5) it is proved that $H^{-1}$ commutes with integer translations, then 


$$
\begin{aligned}
\pi\left(\bar{R}_{w}\left(H^{-1}(x)+v\right)\right) & =R_{w}\left(\pi\left(H^{-1}(x+v)\right)\right) \\
& =R_{w}\left(h^{-1}(\pi(x+v))\right) \\
& =\pi\left(\bar{R}_{w}\left(H^{-1}(x)\right)\right) \\
& =\pi\left(\bar{R}_{w}\left(H^{-1}(x)\right)+v\right) .
\end{aligned}
$$

Doing $z=H^{-1}(x)$, the above equality implies that the rotation about the origin commutes with every integer translation, $\bar{R}_{w}(z+v)=\bar{R}_{w}(z)+v$, which is a contradiction. Note that $T_{m / n} \circ T_{v}=$ $T_{v} \circ T_{m / n}$. Therefore, $g$ is conjugated to $h \circ T_{m / n} \circ h^{-1}$ and it is of finite order $g^{n}=\left(h \circ T_{m / n} \circ h^{-1}\right)^{n}=$ $h \circ\left(T_{m / n}\right)^{n} \circ h^{-1}=I d_{\mathbb{T}^{2}}$.

Let $k m_{1}, k m_{2}, n$ relatively prime with $k<n$. We assume the existence of some lift for which the following rotation vector is realized.

Theorem 3.3.2. Let $f: \mathbb{T}^{2} \longrightarrow \mathbb{T}^{2}$ be a homeomorphism isotopic to identity, and let $\mathcal{O}$ be a period$n$ periodic orbit with rotation vector $v=\frac{k}{n}\left(m_{1}, m_{2}\right)$. The orbit $\mathcal{O}$ is a finite order periodic orbit if and only if there exists a simple closed curve $\gamma: \mathbb{S}^{1} \longrightarrow \mathbb{T}^{2}$ with $\mathcal{O} \subset \gamma$, such that

1. $\operatorname{Hom}(\gamma)=\left(m_{1}, m_{2}\right)$.

2. $f(\gamma) \simeq \gamma($ rel. $\mathcal{O})$.

Proof. Suppose that $\mathcal{O}$ is a period-n periodic orbit of finite order with rotation vector $v=k m / n$. Since $\mathcal{O}$ is a finite order periodic orbit, there exists $g \in \mathrm{Homeo}^{+}\left(\mathbb{T}^{2}\right)$ isotopic to $f$, relative to the periodic orbit $\mathcal{O}$ of finite order, $g^{n}=i d_{\mathbb{T}^{2}}$. By Proposition 3.3.1, $g$ can be chosen conjugate to $T_{v=k m / n}$ induced by the projection of the translation by $v, \bar{T}_{k m / n}: \mathbb{R}^{2} \longrightarrow \mathbb{R}^{2}$. Note that $v=k m / n$ is not unique, there are many vectors $w$ such that rational translation $\bar{T}_{w}$ induce $T_{v}$. Let $g=h \circ T_{k m / n} \circ h^{-1}$.

Let $\tilde{x} \in \mathbb{R}^{2}$ be a lift $x \in \mathcal{O}, \tilde{x}=\pi^{-1}(x)$. Consider the orbit of $\tilde{x}$ by $G=H \circ \bar{T}_{k m / n} \circ H^{-1}$, $\tilde{\mathcal{O}}=\left\{G^{k}(\tilde{x}) \mid k \in \mathbb{Z}\right\}$. Note that $G^{k}(\tilde{x})=\left(H \circ \bar{T}_{k m / n} \circ H^{-1}\right)^{k}(\tilde{x})=H \circ \bar{T}_{k m / n}^{k} \circ H^{-1}(\tilde{x})$. Then, the orbit $\tilde{\mathcal{O}}$ is carried by a coordinate change in a orbit $\mathcal{O}_{v}$ define by translation $\bar{T}_{k m / n}$. Let $\ell: \mathbb{R} \longrightarrow \mathbb{R}^{2}$ be the straight line $\ell \supset \mathcal{O}_{v}$ with direction vector $v=k m / n$. If $\tilde{\gamma}=H(\ell)$, then $\gamma=\pi(\tilde{\gamma})$ is a simple closed curve that contains $\mathcal{O}$. Since $f$ is isotopic to $g=h \circ T_{k m / n} \circ h^{-1}$ relative to $\mathcal{O}$, it follows $f(\gamma) \simeq g(\gamma)($ rel. $\mathcal{O})$. Therefore, $f(\gamma) \simeq \gamma($ rel. $\mathcal{O})$ as we wanted.

Suppose now that $\gamma$ is a simple closed curve such that $\mathcal{O} \subset \gamma$. Assume $f(\gamma) \simeq \gamma($ rel. $\mathcal{O})$. From the Theorem 2.5.11, we can extend the relative isotopy to the entire two-torus. Let $J: \mathbb{T}^{2} \times[0,1] \longrightarrow \mathbb{T}^{2}$ such torus isotopy. Being $f$ isotopy to the identity, call $I: i d_{\mathbb{T}^{2}} \simeq f$ such isotopy. Consider now the composition map $K=J \circ I$. Since $\mathcal{O}$ is a period $n$-periodic orbit with rotation vector $v=k m / n$ for some lift of $f$, we can assume that the composition acts like a translation by $v$. Call $g$ such map (i.e., $g(x)=K(x, 1)$ ). In particular, $g$ is a finite order torus homeomorphism, $g^{n}=i d_{\mathbb{T}^{2}}$. From the isotopy $J$, we see that $f$ is isotopic to $g$ on the entire torus relative to $\mathcal{O}$. Therefore, $\mathcal{O}$ is a finite order periodic orbit. 


\section{Chapter 4}

\section{Finding finite order periodic orbits}

In this chapter for the torus homeomorphism 3.1, we present necessary conditions to find finite order periodic orbits with rotation vector in the interior or at the hypotenuse of the rotation set (right triangle) onto the set of periodic orbits with points in the regions $\boldsymbol{a}_{\mathbf{1}}, \boldsymbol{a}_{\mathbf{3}}, \boldsymbol{a}_{\mathbf{4}}, \boldsymbol{b}_{\mathbf{1}}, \boldsymbol{b}_{\mathbf{2}}$. From [Par05], the set of finite order periodic orbits set which have rational rotation vector in the interior of the rotation triangle is not empty. The Theorem 3.3.2 in the previous section give us a route of how to search for finite order periodic orbits. This is, by relating a periodic orbit with an oriented simple closed curve that contains it, to examine wether under the action of the homeomorphism the image of the curve is isotopic relative to the orbit, to the original curve.

In section 4.1 we will give a preliminary description of how we will find the periodic orbits. For a rotation vector $v=\frac{k}{n}\left(p_{1}, p_{2}\right)$ with $p_{1}, p_{2}, n$ relatively prime and $n \geq k\left(p_{1}+2 p_{2}\right)$, we will start by describing in the two-torus the distribution of the points of periodic orbits that realize the vector $v$, and how $f$ acts in the regions where these points are contained. In 4.2, we will consider periodic orbits with rotation vector $v$ as before and associate to each orbit an oriented simple closed curve with homology $\left(p_{1}, p_{2}\right)$ that contains it. In the Definition 4.2.10 we present the pairs set with which we will work. In particular, a reasonable condition imposed on these pairs in order to the relative isotopy occur, is that in the circular version of the $\left(p_{1}, p_{2}\right)$-curve the periodic orbit points behave topologically like a rigid rotation. In this way, the finite order periodic orbits set will be a subset of the previous set. We will see that a rotation vector can be realized by many finite order periodic orbits.

Given the set of the orbit-curve pairs, in 4.3 we will divide it in two subsets, where one of them contain the orbits that later will be finite order periodic orbits. Since we have the ability to read the relative positions of the points of a periodic orbit and we know where the points are placed, we can detect in which regions these points force folds in the $p_{1}+p_{2}$ arcs that make up the simple closed curve. The folds that we will consider can not deform up to isotopy, the orbit-curve pairs for which we can avoid these folds will be call unfoldable. We also define a special way to embedded the $\left(p_{1}, p_{2}\right)$ curve in the two-torus. Up to isotopy relative to the orbit these simple closed curve can be deformed to monotonous arcs with a unimodal part. Such pairs will be call unimodal, and we will show that if a pair is unfoldable, then this is unimodal.

Done this, we will proof the main result in the Section4.4. We will show that the unfoldable condition implies that the orbits in such pairs are finite order periodic orbits. The next section contains the symbolic description of the chosen orbits in 4.2. In the final section, we describe the computational program that list for a rotation vector $v$ : (a) The sequences of the periodic orbits with rotation vector $v$. (b) The sequence of the relative positions of the orbit points. (c) Number of periodic orbits. (d) Number of fopos . 


\subsection{Outline of the proposal}

Let us consider the torus homeomorphism in 3.1.1 and let $v$ a rotation vector in the interior of the rotation set or in its boundary, this is $v=\frac{k}{n}\left(p_{1}, p_{2}\right)$ with $p_{1}, p_{2}, n$ relatively prime, and $n \geq k\left(p_{1}+2 p_{2}\right)$. If $v$ is realized by a $f$-periodic orbit, we will only consider orbits with points in the interior of the regions: $\boldsymbol{a}_{\mathbf{1}}, \boldsymbol{a}_{\mathbf{3}}, \boldsymbol{a}_{\mathbf{4}}, \boldsymbol{b}_{\mathbf{1}}$, and $\boldsymbol{b}_{\mathbf{2}}$. In particular, if $n=k\left(p_{1}+2 p_{2}\right)$ the orbits with $v=\left(\frac{p_{1}}{q}, \frac{p_{2}}{q}\right)\left(q=p_{1}+2 p_{2}\right)$ only will take points in the regions $\boldsymbol{a}_{\mathbf{1}}, \boldsymbol{a}_{\mathbf{4}}$, and $\boldsymbol{b}_{\mathbf{2}}$. The orbit points are distributed in the following way:

\begin{tabular}{|c|c|c|c|c|}
\cline { 2 - 5 } \multicolumn{1}{c|}{} & $\boldsymbol{b}_{\mathbf{2}}$ & $\boldsymbol{a}_{\mathbf{4}}$ & $\boldsymbol{a}_{\mathbf{1}}$ & $\boldsymbol{a}_{\mathbf{3}} \vee \boldsymbol{b}_{\mathbf{1}}$ \\
\hline $\boldsymbol{n}=\boldsymbol{k q}$ & $k p_{2}$ & $k p_{1}$ & $k p_{2}$ & 0 \\
\hline $\boldsymbol{n}>\boldsymbol{k q}$ & $k p_{2}$ & $k p_{1}$ & $k p_{2}$ & $n-k\left(p_{1}+2 p_{2}\right)$ \\
\hline
\end{tabular}

Now, the images of these $n$ points are completely determined, as follows

$$
\begin{gathered}
a_{3} \stackrel{(0,0)}{\longrightarrow}\left\{\begin{array} { l } 
{ a _ { 3 } , } \\
{ a _ { 4 } }
\end{array} \quad a _ { 1 } \stackrel { ( 0 , 0 ) } { \longrightarrow } \left\{\begin{array} { l } 
{ b _ { 1 } , } \\
{ b _ { 2 } }
\end{array} \quad a _ { 4 } \stackrel { ( 1 , 0 ) } { \longrightarrow } \left\{\begin{array}{l}
a_{1}, \\
a_{3}, \\
a_{4}
\end{array}\right.\right.\right. \\
b_{1} \stackrel{(0,0)}{\longrightarrow}\left\{\begin{array} { l } 
{ b _ { 1 } , } \\
{ b _ { 2 } }
\end{array} \quad b _ { 2 } \stackrel { ( 0 , 1 ) } { \longrightarrow } \left\{\begin{array}{l}
a_{1}, \\
a_{3}, \\
a_{4}
\end{array}\right.\right.
\end{gathered}
$$

where the pairs $(0,0),(1,0),(0,1)$ represent the change of domains in the universal cover, $\mathbb{R}^{2}$. From the action of the homeomorphism we can also recognize the places in the $\boldsymbol{a}, \boldsymbol{b}$ strips, where the orbit points and their images are located (see figures 3.4, 4.1(b)(c)). Notice that for $\ell=n-k\left(p_{1}+p_{2}\right)>0$, a fixed rotation vector $v$ can be realized by many different periodic orbits. We will show that, such orbits have the same number of points in $\boldsymbol{a}_{\mathbf{1}}, \boldsymbol{a}_{\mathbf{4}}, \boldsymbol{b}_{\mathbf{2}}$ and share in the symbolic sequence the "same" location. All them differ in the number of terms between $\boldsymbol{a}_{\mathbf{3}}, \boldsymbol{b}_{\mathbf{1}}$ and their location in the symbolic sequence. Every period- $n$ periodic orbit will be described by a sequence in $\left\{a_{1}, a_{3}, a_{4}, b_{1}, b_{2}\right\}^{\mathbb{N}}$, for which its itinerary satisfies the relations given in (4.2). 


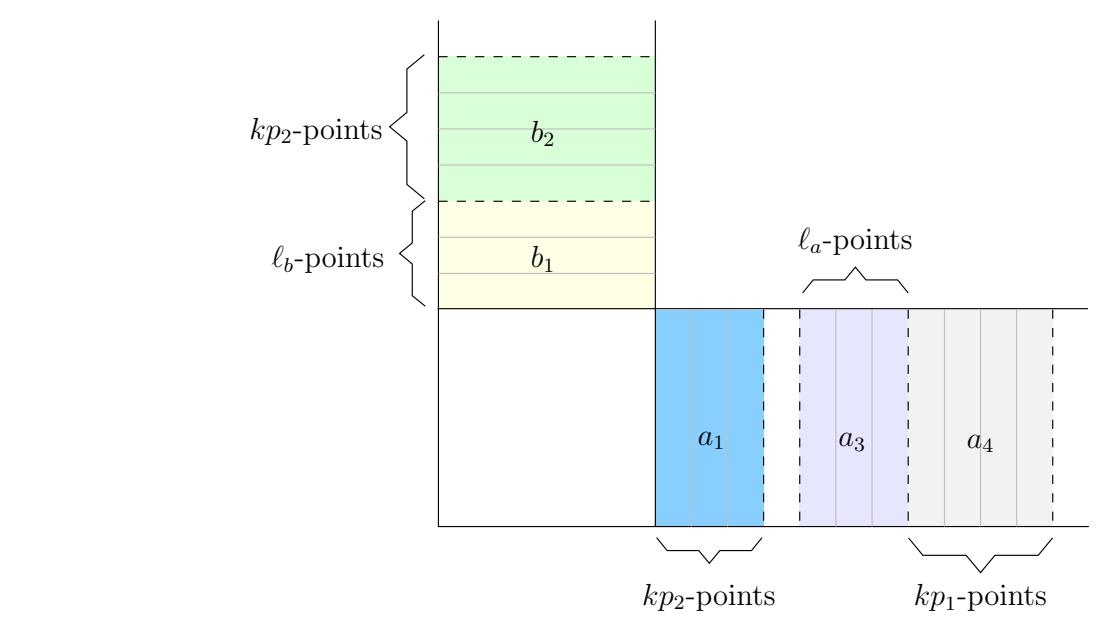

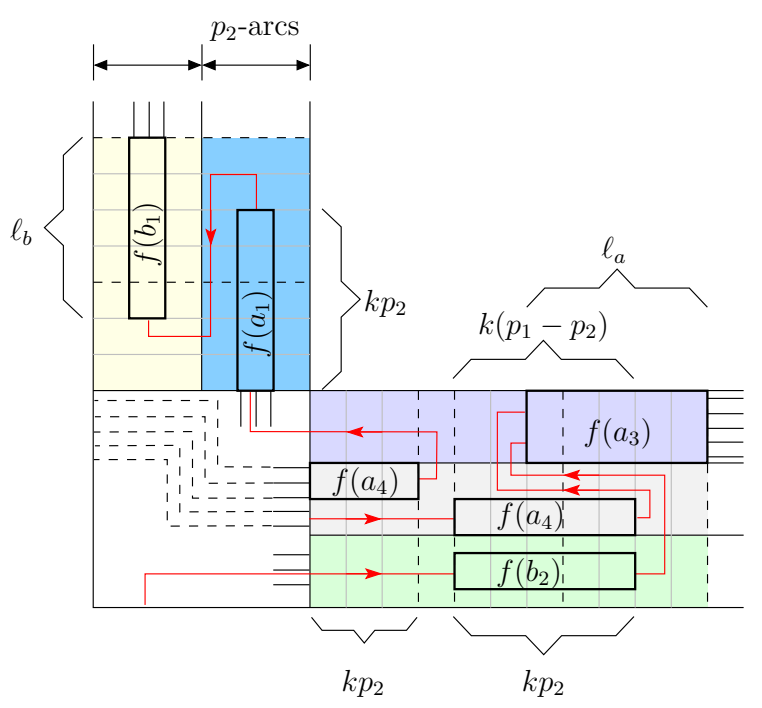

(b) $p_{1}>p_{2}$

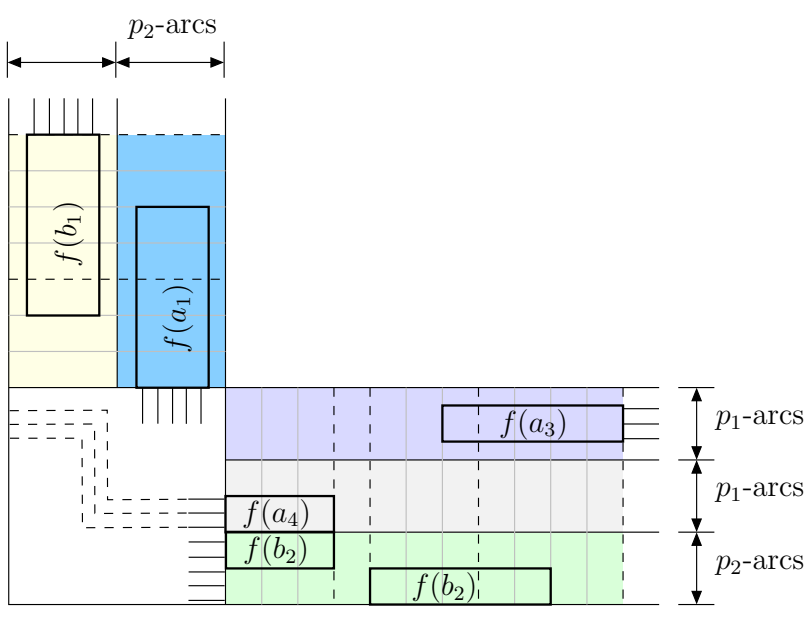

(c) $p_{1}<p_{2}$

Figure 4.1: (a) The fibered zones represent the places where the orbit takes values, each point is located in a different leaf. (b) (c) By colors we can identify the action of $f$ on the regions $\boldsymbol{a}_{\mathbf{1}}, \boldsymbol{a}_{\mathbf{3}}, \boldsymbol{a}_{\mathbf{4}}, \boldsymbol{b}_{\mathbf{1}}$, and $\boldsymbol{b}_{\mathbf{2}}$. The black boxes represent the place where the orbit points meets with the arcs of $\gamma$. Up to isotopy the a-arcs pass inside the a-strip containing the $a_{3}$ 's, $a_{4}$ 's orbit points, and the b-arcs run through the $a_{1}$ 's, $b_{1}$ 's, $b_{2}$ 's points.

Fixed a periodic orbit $\mathcal{O}$ with rotation vector $v=\frac{k}{n}\left(p_{1}, p_{2}\right)$ as before. Theorem 3.3.2 suggest us to look for a simple closed curve $\gamma$ with homological direction $\left(p_{1}, p_{2}\right)$ containing $\mathcal{O}$, such that up to isotopy relative to the orbit the simple closed curve $\gamma$ is invariant by the action of the homeomorphism $f$. We will start by describing such simple closed curve, and we will give conditions for $\gamma$ to go through the orbit points. Consider $\gamma: \mathbb{S}^{1} \longrightarrow \mathbb{T}^{2}$ a oriented (clockwise, say) simple closed curve with homology $\operatorname{Hom}(\gamma)=\left(p_{1}, p_{2}\right)$ divide into $p_{1}+p_{2}$ arcs, which up to isotopy correspond to $p_{2}$ meridian arcs crossing the vertical band $\boldsymbol{b}$, and $p_{1}$ parallel arcs crossing the horizontal band $\boldsymbol{a}$ (see the figure 4.2). 

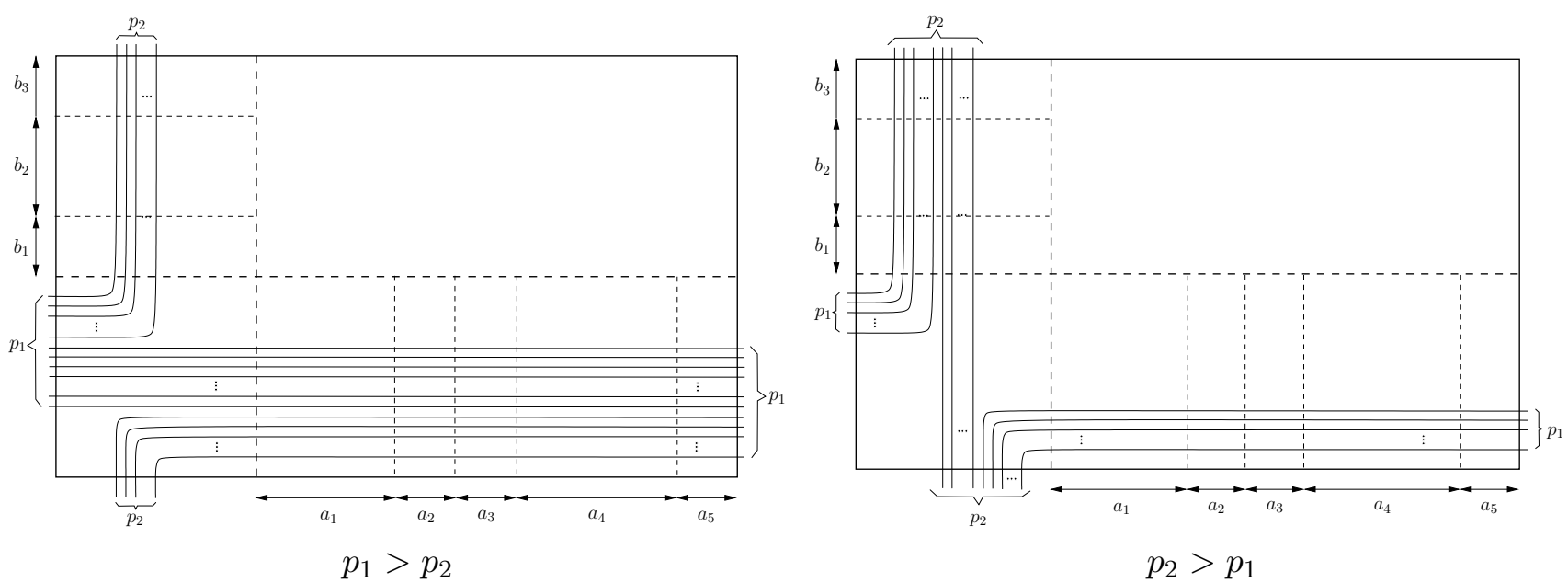

Figure 4.2: Representation of the $\left(p_{1}, p_{2}\right)$ simple closed curve $\gamma$. The curve is divided in $p_{1}$ parallel arcs, and $p_{2}$ meridian arcs.

Number the simple arcs from 0 to $p_{1}+p_{2}-1$, the $a$-arcs (arcs on $\boldsymbol{a}$-strip) from 0 to $p_{1}-1$, and the $b$-arcs (arcs on $\boldsymbol{b}$-strip) from $p_{1}$ to $p_{1}+p_{2}-1$ (see figure 4.3). The $\operatorname{arcs} \gamma_{i}\left(0 \leqslant i \leqslant p_{1}+p_{2}-1\right)$ that divide $\gamma$, get joined in the junction by $\bmod \left(p_{1}+p_{2}\right)$ addition (see expression 4.3$)$. Notice that for two continuous arcs $\gamma_{i}, \gamma_{i+1}$ in the $\boldsymbol{a}$-strip or $\boldsymbol{b}$-strip, the $\gamma_{i+1}$ arc always runs through the strip to the left of $\gamma_{i}$ arc.

$$
\begin{array}{ccccccc}
\gamma_{0} & \gamma_{1} & \cdots & \gamma_{p_{1}} & \gamma_{p_{1}+1} & \cdots & \gamma_{p_{1}+p_{2}-1} \\
\downarrow & \downarrow & & \downarrow & \downarrow & & \downarrow \\
\gamma_{p_{2}} & \gamma_{1+p_{2}} & \cdots & \gamma_{p_{1}+p_{2}} & \gamma_{1} & \cdots & \gamma_{p_{2}-1}
\end{array}
$$

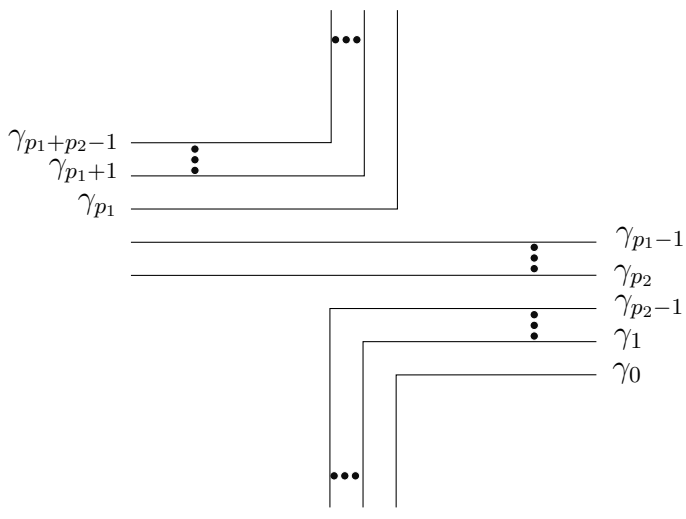

$p_{1}>p_{2}$

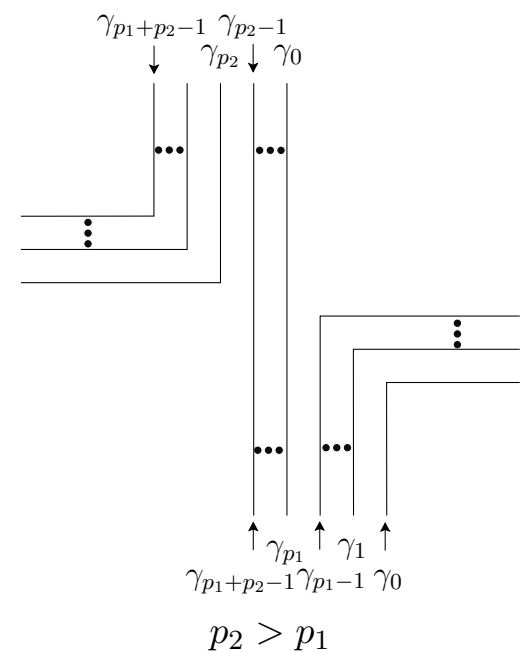

$p_{2}>p_{1}$

Figure 4.3: The simple arcs are numerate according to their naturally modular behavior.

Once $\left(p_{1}, p_{2}\right)$ (relatively prime) are chosen, the curve $\gamma$ is determined and the way the $a$-arcs and $b$-arcs follow each other around $\gamma$. Now, we need to decide how the $p_{1}+p_{2}$ arcs run through the periodic orbit points. The points in $\mathcal{O}$ are placed in different leafs in the interior of the regions $\boldsymbol{a}_{\mathbf{1}}, \boldsymbol{a}_{\mathbf{3}}, \boldsymbol{a}_{\mathbf{4}}, \boldsymbol{b}_{\mathbf{1}}, \boldsymbol{b}_{\mathbf{2}}$ of the fibered subsurface $\mathbb{X}$, and each of them will be contained in an $a$-arc, or $b$-arc. Let $\ell_{a}, \ell_{b}$ be orbit points in $\boldsymbol{a}_{\mathbf{3}}, \boldsymbol{b}_{\mathbf{1}}$ respectively (or one of them), we will assume that the $p_{1} a$-arcs contain $\ell_{a}$ points in $\boldsymbol{a}_{\mathbf{3}}, k p_{1}$ points in $\boldsymbol{a}_{\mathbf{4}}$, and the $p_{2} b$-arcs contain $\ell_{b}$ points in $\boldsymbol{b}_{\mathbf{1}}, k p_{2}$ points in $\boldsymbol{b}_{\mathbf{2}}$ (see figure 4.1(a)). Since only the points in the region $\boldsymbol{a}_{\mathbf{1}}$ go from $\boldsymbol{a}$-strip to $\boldsymbol{b}$-strip 
(see the blue block), these points function as a link between the two strips. This passage is given in the following way

$$
\left\{\begin{array} { l } 
{ \tilde { a } _ { 4 } , } \\
{ \tilde { b } _ { 2 } }
\end{array} \stackrel { ( 0 , 1 ) } { \underset { ( 1 , 0 ) } { \longrightarrow } } \quad \tilde { a } _ { 1 } \quad \underset { ( 0 , 0 ) } { \stackrel { ( 0 , 0 ) } { \longrightarrow } } \left\{\begin{array}{l}
\tilde{b}_{1}, \\
\tilde{b}_{2}
\end{array}\right.\right.
$$

We note that in a fundamental domain $\tilde{f}\left(\tilde{a}_{1}\right)$ do not change of domain for lifts $\tilde{f}, \tilde{a}_{1}$ to $\mathbb{R}^{2}$, it suggests us to deform isotopically the $p_{2}$ arcs that go to vertical strip $\boldsymbol{b}$, first going through $\boldsymbol{a}_{\mathbf{1}}$ before going to $\boldsymbol{b}$ (see figure 4.4). Up to isotopy we will assume that the $a$-arcs that pass through $\boldsymbol{a}$-strip only intersect $\boldsymbol{a}$, and the $b$-arcs are allowed to make a unimodal detour through $\boldsymbol{a}_{\mathbf{1}}$ before go to $\boldsymbol{b}$.

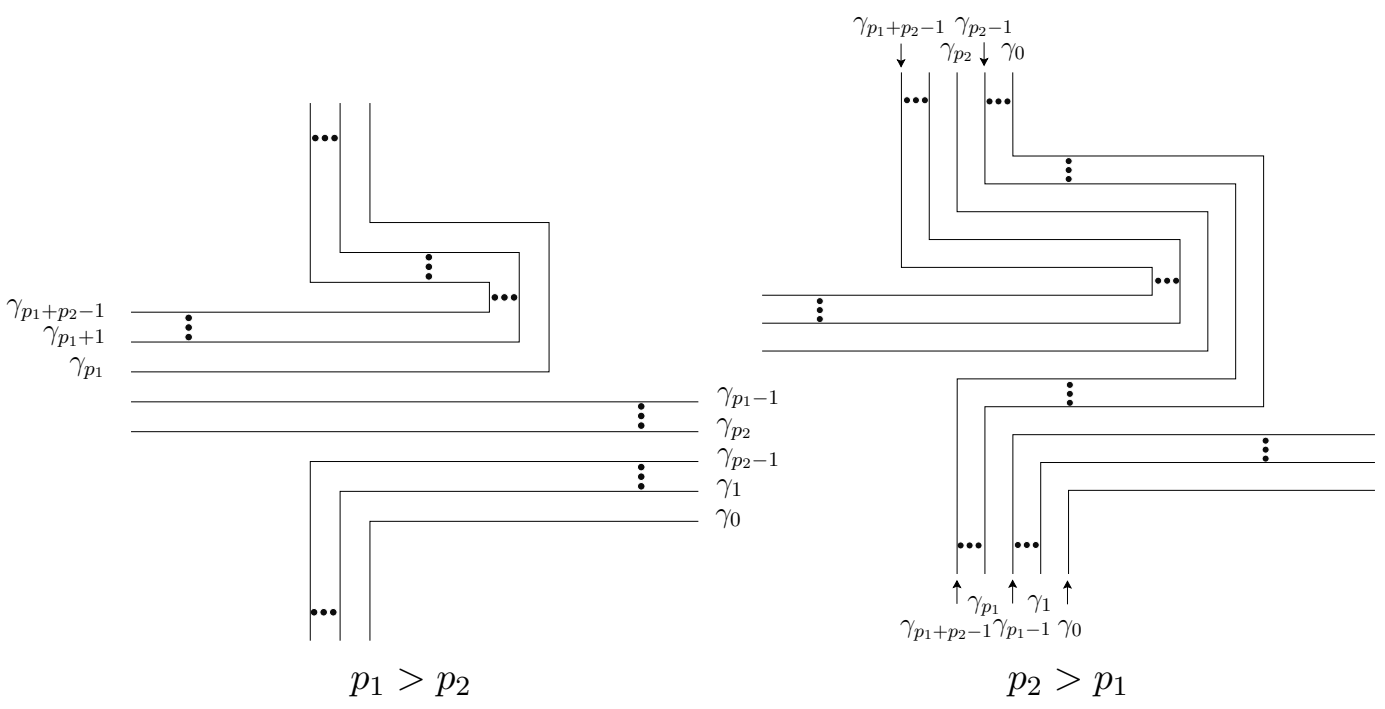

Figure 4.4: Homotopic deformation of the curve $\gamma$ on the region $\boldsymbol{a}_{\mathbf{1}}$.

Our assumption is not arbitrary, it follows from careful observation of how the homeomorphism acts onto the points in $\mathcal{O}$ and the arcs of $\gamma$. On the one hand, the Figure 4.1 illustrate with colors the behavior of the periodic orbit points, we use the boxes to indicate the places of the points. For example in the case $p_{1}>p_{2}$, the $f$-image of the $k p_{1}$ orbit periodic points in the gray region $\boldsymbol{a}_{\mathbf{4}}$ are distributed in $k p_{2}, k\left(p_{1}-p_{2}\right)$ points between the regions $\boldsymbol{a}_{\mathbf{1}}$, and $\boldsymbol{a}_{\mathbf{3}}, \boldsymbol{a}_{\mathbf{4}}$ respectively (see row one, table 4.1). In summary,

\begin{tabular}{|c|c|c|c|c|}
\hline \multirow{3}{*}{$\begin{array}{ll}x & \longmapsto \\
a_{4} & \\
k p_{1} & \end{array}$} & \multicolumn{4}{|c|}{$f(x)$} \\
\hline & \multicolumn{2}{|r|}{$p_{1}>p_{2}$} & \multirow{2}{*}{\multicolumn{2}{|c|}{$\begin{array}{c}p_{2}>p_{1} \\
a_{1} \\
k p_{1}\end{array}$}} \\
\hline & $\begin{array}{l}a_{1} \\
k p_{2}\end{array}$ & $\begin{array}{r}a_{3} \wedge a_{4} \\
k\left(p_{1}-p_{2}\right)\end{array}$ & & \\
\hline $\begin{array}{l}b_{2} \\
k p_{2}\end{array}$ & & $\begin{array}{c}a_{3} \wedge a_{4} \\
k p_{2}\end{array}$ & & $\begin{array}{r}a_{3} \wedge a_{4} \\
k\left(p_{2}-p_{1}\right)\end{array}$ \\
\hline $\begin{array}{l}a_{1} \\
k p_{2}\end{array}$ & \multicolumn{4}{|c|}{$\begin{array}{c}b_{1} \wedge b_{2} \\
k p_{2}\end{array}$} \\
\hline $\begin{array}{l}a_{3} \\
\ell_{a}\end{array}$ & \multicolumn{4}{|c|}{$\begin{array}{c}a_{3} \wedge a_{4} \\
\ell_{a}\end{array}$} \\
\hline $\begin{array}{l}b_{1} \\
\ell_{b}\end{array}$ & \multicolumn{3}{|c|}{$b_{1} \wedge b_{2}$} & $\ell_{b}$ \\
\hline
\end{tabular}

Table 4.1: Distribution by regions of the periodic orbit points under the action of the homeomorphism $f$. 
In the other hand, from Definition 3.1.1 we know how $f$ expands the $\gamma$ curve, hence its arcs. In particular, $f$ preserve the order of the orbit points in the horizontal and vertical strips (the points in $\boldsymbol{a}_{\mathbf{1}}, \boldsymbol{b}_{\mathbf{2}}$ change from one strip to the other, but the order between their orbit points is preserved), and also preserve the way as two arcs run through the strips, this is the arc $f\left(\gamma_{i+1}\right)$ remains to the left of $f\left(\gamma_{i}\right)$ arc. Using the numbering in (4.3), we can identify the pieces of the arcs in $f(\gamma)$ and $\gamma$ that must overlap for the relative isotopy to occur (see figure 4.5, this illustration allows us to identify the route and the index of the arcs, this does not explain how the arcs move within the strips or pass through the orbit points). Observe for example onto the $\boldsymbol{a}$-strip $\left(p_{1}>p_{2}\right)$ that the image under $f$ of the $b$-arcs with index from $p_{1}$ to $p_{1}+p_{2}-1$ in the $\boldsymbol{b}$-strip, together to the image of the $a$-arcs with index from 0 to $p_{1}-1$ in $\boldsymbol{a}$-strip, follow the same route that the $p_{1}+p_{2} \operatorname{arcs}$ above with indices from 0 to $p_{1}+p_{2}-1$. Intuitively, if we have any hope that relative isotopy can occur, we need that:

$$
f\left(\gamma_{i}\right) \simeq \gamma_{i}\left(\operatorname{rel} P_{b}\right) ; \quad p_{1} \leq i \leq p_{1}+p_{2}-1(\boldsymbol{b}-\text { strip }), \quad P_{b}=\mathcal{O} \bigcap\left(\boldsymbol{b}_{\mathbf{1}} \cup \boldsymbol{b}_{\mathbf{2}}\right),
$$

and

$$
f\left(\gamma_{i}\right) \simeq \gamma_{\left(i+p_{2}\right) \bmod \left(p_{1}+p_{2}\right)}\left(\operatorname{rel} P_{a}\right) ; \quad 0 \leq i \leq p_{1}+p_{2}-1(\boldsymbol{a}-\operatorname{strip}), \quad P_{a}=\mathcal{O} \bigcap\left(\boldsymbol{a}_{\mathbf{1}} \cup \boldsymbol{a}_{\mathbf{3}} \cup \boldsymbol{a}_{\mathbf{4}}\right)
$$

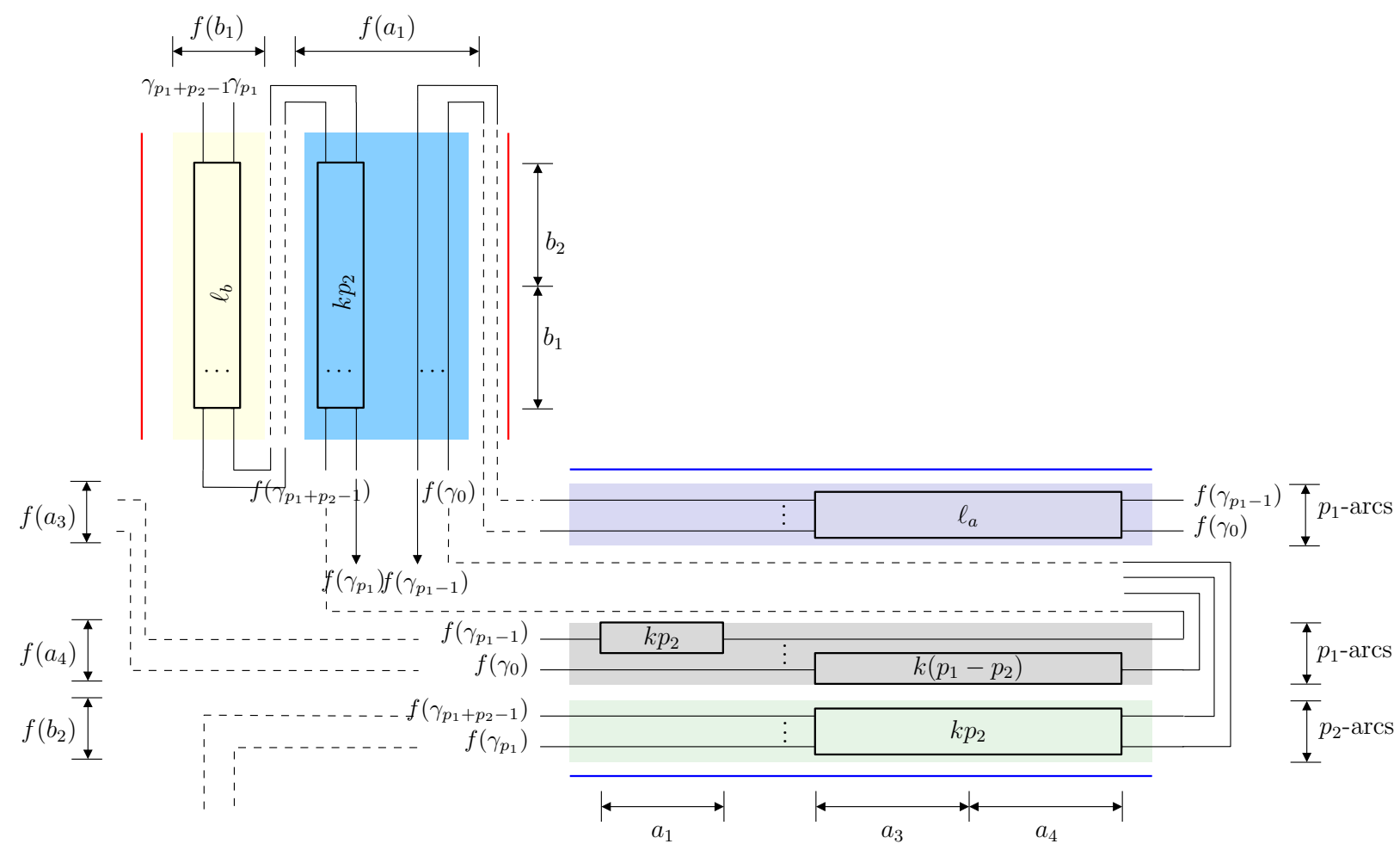

Figure 4.5: Action of the homeomorphism on simple closed curve $\gamma$ for $p_{1}>p_{2}$. The arcs in the picture just represent how them are ordered, and the bold boxes represent the places where the arcs meet the periodic orbit points. Note that the curve can be embedded in many different way runs through the orbits points.

Continuing with the example of the $a_{4}$ 's points for $p_{1}>p_{2}$, by (4.3) the $a$-arcs with indices from 0 to $p_{1}-p_{2}-1$ (i.e., those $a$-arcs that are connected to another $a$-arcs) will pass through $k\left(p_{1}-p_{2}\right)$ points between the regions $\boldsymbol{a}_{\mathbf{3}}, \boldsymbol{a}_{\mathbf{4}}$, and the $a$-arcs with indices from $p_{1}-p_{2}$ to $p_{1}-1$ (i.e., those $a$-arcs that are connected to $b$-arcs) will pass through the $k p_{2}$ points in the region $\boldsymbol{a}_{\mathbf{1}}$ (see row one, table 4.2). In the Figure 4.1(b), we illustrate with a red strand the route that the arcs follows inside the strips. In summary, 


\begin{tabular}{|c|c|c|c|c|c|c|c|}
\hline \multicolumn{8}{|c|}{$p_{1}>p_{2}$} \\
\hline \multicolumn{4}{|c|}{$\gamma_{i}$} & \multicolumn{4}{|c|}{$\gamma_{\delta=\left(i+p_{2}\right) \bmod \left(p_{1}+p_{2}\right)}$} \\
\hline $0 \leq i \leq p_{1}-1$ & $a-\operatorname{arcs}$ & $a_{4}$ & $k p_{1}$ & $\begin{array}{c}p_{2} \leq \delta \leq p_{1}-1 \\
p_{1} \leq \delta \leq p_{1}+p_{2}-1\end{array}$ & $\begin{array}{l}a-\operatorname{arcs} \\
b-\operatorname{arcs}\end{array}$ & $\begin{array}{c}a_{3} \wedge a_{4} \\
a_{1}\end{array}$ & $\begin{array}{c}k\left(p_{1}-p_{2}\right) \\
k p_{2}\end{array}$ \\
\hline$p_{1} \leq i \leq p_{1}+p_{2}-1$ & $b-\operatorname{arcs}$ & $b_{2}$ & $k p_{2}$ & $0 \leq \delta \leq p_{2}-1$ & $a-\operatorname{arcs}$ & $a_{3} \wedge a_{4}$ & $k p_{2}$ \\
\hline \multicolumn{8}{|c|}{$p_{1}<p_{2}$} \\
\hline \multicolumn{4}{|c|}{$\gamma_{i}$} & \multicolumn{4}{|c|}{$\gamma_{\delta=\left(i+p_{2}\right) \bmod \left(p_{1}+p_{2}\right)}$} \\
\hline$p_{1} \leq i \leq p_{1}+p_{2}-1$ & $b-\operatorname{arcs}$ & $b_{2}$ & $k p_{2}$ & $\begin{array}{c}0 \leq \delta \leq p_{1}-1 \\
p_{1} \leq \delta \leq p_{2}-1\end{array}$ & $\begin{array}{l}a-\operatorname{arcs} \\
b-\operatorname{arcs}\end{array}$ & $\begin{array}{c}a_{3} \wedge a_{4} \\
a_{1}\end{array}$ & $\begin{array}{c}k p_{1} \\
k\left(p_{2}-p_{1}\right)\end{array}$ \\
\hline $0 \leq i \leq p_{1}-1$ & $b-\operatorname{arcs}$ & $a_{4}$ & $k p_{1}$ & $p_{2} \leq \delta \leq p_{1}+p_{2}-1$ & $b-\operatorname{arcs}$ & $a_{1}$ & $k p_{1}$ \\
\hline \multicolumn{8}{|c|}{$p_{1}>p_{2} \wedge p_{1}<p_{2}$} \\
\hline \multicolumn{4}{|c|}{$\gamma_{i}$} & \multicolumn{4}{|c|}{$\gamma_{i}$} \\
\hline $0 \leq i \leq p_{1}-1$ & $a-\operatorname{arcs}$ & $a_{3}$ & $\ell_{a}$ & $0 \leq i \leq p_{1}-1$ & $a-\operatorname{arcs}$ & $a_{3} \wedge a_{4}$ & $\ell_{a}$ \\
\hline$p_{1} \leq i \leq p_{1}-p_{2}-1$ & $b-\operatorname{arcs}$ & $a_{1}$ & $k p_{2}$ & $p_{1} \leq i \leq p_{1}-p_{2}-1$ & $b-\operatorname{arcs}$ & $b_{1} \wedge b_{2}$ & $k p_{2}$ \\
\hline$p_{1} \leq i \leq p_{1}-p_{2}-1$ & $b-\operatorname{arcs}$ & $b_{1}$ & $\ell_{b}$ & $p_{1} \leq i \leq p_{1}-p_{2}-1$ & $b-\operatorname{arcs}$ & $b_{1} \wedge b_{2}$ & $\ell_{b}$ \\
\hline
\end{tabular}

Table 4.2: The arcs go through the orbit points respecting the behavior of the blocks in table 4.1, and the way they are linked following 4.3.

Although we can recognize the places where the simple closed curve meets the orbit, it is not enough to know how the curve passes through the points. Even with the above conditions the simple closed curve can be embedded in the torus bands in many different way.

Once we have decided the route of the $\left(p_{1}, p_{2}\right)$-curve in the cloud of orbit points in strips $\boldsymbol{a}, \boldsymbol{b}$, we can talk about the location of the $n=k\left(p_{1}+2 p_{2}\right)+\ell$ points in the arcs of the simple closed curve. For this, we consider the circular version of $\gamma$, drawing a circle divided into $p_{1}+p_{2}$ arcs, by (4.3), we know that arcs go across $\boldsymbol{a}$-strip (i.e., go around a longitude), which arcs go across $\boldsymbol{b}$-strip (i.e., go around a meridian) and how $a$-arcs and $b$-arcs follow each other around $\gamma$. Additionally, from the Tables 4.1, 4.2 we also know in which arcs the $a_{1}$ 's, $a_{3}$ 's, $a_{4}$ 's, $b_{1}$ 's, $b_{2}$ 's points can be placed. Therefore, we just need to decide how many points of each type are in each arc. For this, we can distribute their $n$ points in the circular version of $\gamma$ make them evenly spaced, a $2 \pi / n$ (clockwise).

We are looking for finite order periodic orbits, from Theorem 3.3.2 is necessary and sufficient the invariance of $\gamma$ up to isotopy relative to the periodic orbit $\mathcal{O}$ by $f$. So such a relative isotopy must satisfies the Equations (4.4) and since the action of $f$ preserves the order given to the points in the $a$-arcs, $b$-arcs, a translation of the points occurs in the direction induced in the curve $\gamma$. Then, our second choice is to take a periodic orbit which is conjugate to a rotation by angle $2 k \pi / n$ in the circular representation of $\gamma$, preserving the properties defined above. We will say that a periodic orbit with this quality is compatible with a $2 k \pi / n$-rotation, and call the set of such periodic orbits by $\Omega$. We finalize this paragraph saying that the previous rules on the periodic orbits no guarantee the relative isotopy in $\gamma$, but it defines the set of periodic orbits that have the possibility of being fopos.

Main Theorem Let $(\mathcal{O}, \gamma)$ be a compatible orbit-curve pair. If $(\mathcal{O}, \gamma)$ is unfoldable, then $\mathcal{O}$ is a finite order periodic orbit.

\subsection{Compatible orbit-curve pair}

The proof of the following propositions follows directly from the Definition 3.1.1.

Definition 4.2.1. Let $R \subset \mathbb{T}^{2}$ be a subset of the two-torus. We say that $R$ is a closed rectangular region if its lift to the universal cover $\mathbb{R}^{2}$ is a region of the form $\left[x_{1}, y_{1}\right] \times\left[x_{2}, y_{2}\right]$.

Proposition 4.2.2. The sets $\boldsymbol{a}, \boldsymbol{b}, \boldsymbol{a}_{\boldsymbol{i}}(i=1, \ldots, 5)$ and $\boldsymbol{b}_{\boldsymbol{j}}(j=1,2,3)$ in the Definition 3.1 .1 are rectangular regions. 
Corollary 4.2.3. The unions

$$
\bigcup_{\substack{i=l \\(l \leq m)}} \boldsymbol{a}_{\boldsymbol{i}} \subset \boldsymbol{a} \quad(l, m \in\{1, \ldots 5\}) \quad \text { and } \bigcup_{\substack{j=l^{\prime} \\\left(l^{\prime} \leq m^{\prime}\right)}} \boldsymbol{b}_{\boldsymbol{j}} \subset \boldsymbol{b} \quad\left(l^{\prime}, m^{\prime} \in\{1,2,3\}\right),
$$

are rectangular regions.

Remark. From now on, we will refer to the sets in Proposition 4.2.2 and 4.2.3 as rectangular regions or just regions.

Notation. Let $I^{2}=\left[x_{1}, y_{1}\right] \times\left[x_{2}, y_{2}\right]$. We denote its boundary as $\partial\left(I^{2}\right)=\bigcup_{i=1}^{4} l_{i}$ such that

$$
\begin{array}{llll}
l_{1}=\left[x_{1}, y_{1}\right] \times\left\{x_{2}\right\} & & \\
l_{2}=\left[x_{1}, y_{1}\right] \times\left\{y_{2}\right\} \\
l_{3}=\left\{x_{1}\right\} \times\left[x_{2}, y_{2}\right] \\
l_{4}=\left\{y_{1}\right\} \times\left[x_{2}, y_{2}\right]
\end{array} \quad \begin{array}{ll}
\text { in }\left(I^{2}\right) & =l_{2} \cup l_{4}
\end{array} .
$$

Let $R$ a rectangular region. We will write the sides of $R$ using the map projection, as follows: $\pi\left(l_{i}\right)=l_{i}^{R}(i=1, . ., 4)$ and $\pi\left(\partial^{\text {in }}\left(I^{2}\right)\right)=\partial^{\text {in }}(R)=l_{2}^{R} \cup l_{4}^{R}, \pi\left(\partial^{\text {out }}\left(I^{2}\right)\right)=\partial^{\text {in }}(R)=l_{1}^{R} \cup l_{3}^{R}$ respectively.

Notation. Let $\alpha$ be a cross-cut in the closed rectangular region $R$. We denote by $R^{r i}(\alpha), R^{l e}(\alpha)$ to the closed topological disks to the right (ri) and left (le) from $\alpha$.

Definition 4.2.4. Let $\alpha_{1}, \alpha_{2}$ be a cross-cuts in the region $R$. We will say that $\alpha_{1}$ is relation with

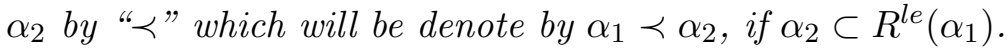

REMARK. The relation " $\prec$ " is a total order in $R$.

Proposition 4.2.5. Let $f$ be as Definition 3.1.1 and $(\mathcal{O}, \gamma)$ a compatible orbit-curve pair. Then, $f$ preserves the relation “ $\prec$ ", as follows:

(i) $f\left(l_{2}^{b_{2}}\right) \prec f\left(l_{4}^{b_{2}}\right) \prec f\left(l_{1}^{a_{4}}\right) \prec f\left(l_{3}^{a_{4}}\right) \prec f\left(l_{1}^{a_{3}}\right) \prec f\left(l_{3}^{a_{3}}\right)$ on the region $\boldsymbol{a}$,

(ii) $f\left(l_{1}^{a_{1}}\right) \prec f\left(l_{3}^{a_{1}}\right) \prec f\left(l_{2}^{b_{1}}\right) \prec f\left(l_{4}^{b_{1}}\right) \prec f\left(l_{1}^{a_{3}}\right)$ on the region $\boldsymbol{b}$.

Proposition 4.2.6. Let $\partial R$ the boundary of a rectangular region. Then

(i) $f\left(\partial^{\text {in }}\left(\boldsymbol{a}_{\mathbf{1}}\right)\right) \subset \partial^{\text {out }}(\boldsymbol{b})$,

(ii) $f\left(\partial^{\text {out }}\left(\boldsymbol{b}_{\mathbf{2}}\right)\right) \subset \partial^{\text {in }}(\boldsymbol{a})$,

(iii) $f\left(\partial^{i n}\left(\boldsymbol{a}_{3}\right)\right) \subset \partial^{i n}(\boldsymbol{a})$,

(iv) $f\left(\partial^{i n}\left(\boldsymbol{a}_{4}\right)\right) \subset \partial^{i n}(\boldsymbol{a})$,

(v) $f\left(\partial^{\text {out }}\left(\boldsymbol{b}_{\mathbf{1}}\right)\right) \subset \partial^{\text {out }}(\boldsymbol{b})$.

Remark. Let $R=a_{i}(i=3, \ldots, 5)$ or $R=b_{2}$. From the Definition 3.1.1, $f(R) \cap \boldsymbol{a}$ and this has a piece outside of $\boldsymbol{a}$ contained in the disk $\mathbb{V}$. Since the orbit points are contained in the interior of $R$, there exist leaves in $R$ close enough to the frontier for which the inclusions (ii)(iii)(iv) can occur in the above proposition. Similarly, for $R=a_{i}(i=1,2)$ or $R=b_{j}(j=1,3)$ the inclusions (i)(v) occur on $\boldsymbol{b}$.

Corollary 4.2.7. The images $f\left(\boldsymbol{a}_{\boldsymbol{i}}\right)(i=1, \ldots, 5)$ and $f\left(\boldsymbol{b}_{\boldsymbol{j}}\right)(j=1,2,3)$ are closed rectangular regions. 
Convention. From now on, we will assume that $n, p_{1}, p_{2}$ are relatively prime with $n \leq k\left(p_{1}+2 p_{2}\right)$.

Definition 4.2.8. Let $f: \mathbb{T}^{2} \longrightarrow \mathbb{T}^{2}$ be as in Definition 3.1.1. We will call $\mathcal{O}$ to the $f$-periodic orbit of period $n$ with rotation vector $v=\frac{k}{n}\left(p_{1}, p_{2}\right)$ which has points in the interior of the regions $a_{1}, a_{3}, a_{4}, b_{1}$, and $b_{2}$.

Convention. We will consider orbits $\mathcal{O}$ in which the $a_{3}$ 's points only have image in $\boldsymbol{a}_{4}$ by $f$.

Notation. We denote by

$$
\Sigma_{a_{i}}=\boldsymbol{a}_{\boldsymbol{i}}{ }^{\circ} \cap \mathcal{O} \quad(i=1,3,4) ; \quad \Sigma_{b_{j}}=\boldsymbol{b}_{\boldsymbol{j}}{ }^{\circ} \cap \mathcal{O} \quad(j=1,2) .
$$

and

$$
\Sigma_{a}=\bigcup_{i=1,3,4} \Sigma_{a_{i}}, \Sigma_{b}=\bigcup_{j=1,2} \Sigma_{b_{j}}
$$

Definition 4.2.9. Let $\mathbb{X} \subset \mathbb{T}^{2}$ be the fibered subsurface defined in 3.1.1 and let $\gamma: \mathbb{S}^{1} \longrightarrow \mathbb{X}^{\circ}$ be a simple closed curve for which $\operatorname{Hom}(\gamma)=\left(p_{1}, p_{2}\right)$. We say that $\gamma$ is a $\left(\boldsymbol{p}_{\mathbf{1}}, \boldsymbol{p}_{\mathbf{2}}\right)$-curve, if $\gamma$ contains $p_{1}+p_{2}$ disjoint simple closed arcs $\gamma_{i}, i=0, \ldots, p_{1}+p_{2}-1$, as follows

(i) The pieces of the simple arcs $\gamma_{i} \subset \gamma \cap \boldsymbol{a}$ are such that, $\left(\gamma_{i} \cap \boldsymbol{a}\right)^{\circ} \subset a^{\circ}$ with $\partial\left(\gamma_{i} \cap \boldsymbol{a}\right) \subset l_{4}^{a} \cup l_{2}^{a}$ $\left(i=0, \ldots, p_{1}-1\right),\left(\gamma_{i} \cap \boldsymbol{a}_{\mathbf{1}}\right)^{\circ} \subset \boldsymbol{a}_{\mathbf{1}}^{\circ}$ with $\partial\left(\gamma_{i} \cap \boldsymbol{a}_{\mathbf{1}}\right) \subset l_{4}^{a_{1}}\left(i=p_{1}, \ldots, p_{1}+p_{2}-1\right)$, and $\left(\gamma_{0} \cap \boldsymbol{a}\right) \prec \cdots \prec\left(\gamma_{p_{1}+p_{2}-1} \cap \boldsymbol{a}\right)$;

(ii) The pieces of the simple arcs $\gamma_{i} \subset \gamma \cap \boldsymbol{b}$ are such that $\partial\left(\gamma_{i} \cap \boldsymbol{b}\right) \subset l_{1}^{b} \cup l_{3}^{b}\left(i=p_{1}, \ldots, p_{1}+p_{2}-1\right)$;

(iii) The $\gamma_{i} \cap\left(\boldsymbol{a}_{\mathbf{1}} \cup \boldsymbol{b}\right)\left(i=p_{1}, \ldots, p_{1}+p_{2}-1\right)$ pieces in (i) and (ii) are connected through $\mathbb{V}$; and

(iv) Each $\gamma_{\delta}$ is the arc following to $\gamma_{i}$ for $\delta=\left(i+p_{2}\right) \bmod \left(p_{1}+p_{2}\right)$ addition module (see figure 4.4).

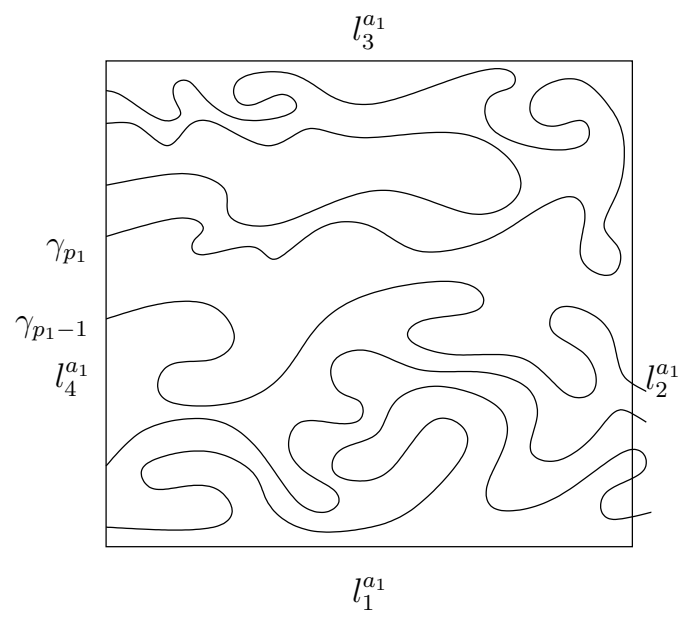

Figure 4.6: The simple closed arcs on $\boldsymbol{a}_{\mathbf{1}}$ region.

Convention. We refer to the simple $\operatorname{arcs} \gamma_{i} \cap \boldsymbol{a}$ just as $\gamma_{i}$ for $\left(i=0, \ldots, p_{1}-1\right)$. The arc $\gamma_{i}=$ $\gamma \cap\left(\boldsymbol{a}_{\mathbf{1}} \cup \boldsymbol{b}\right)$ is not strictly a simple arc since one piece is contained in the disk $\mathbb{V}$. We assume that $\gamma_{i}\left(i=p_{1}, \ldots, p_{1}+p_{2}-1\right)$ is the simple arc that first goes through $\left[\boldsymbol{a}_{\mathbf{1}}\right]^{\circ}$ which enters and leaves by $l_{1}^{a}$ then it passes through $\mathbb{V}$ and continuous to $\boldsymbol{b}$.

Notation. We denote by

$$
\Gamma_{a}=\gamma_{0} \cup \cdots \cup \gamma_{p_{1}-1} \quad, \quad \Gamma_{b}=\gamma_{p_{1}} \cup \cdots \cup \gamma_{p_{1}+p_{2}-1} .
$$


Notation. We denote the relative positions of the points $z \in \Sigma_{a} \cup \Sigma_{b}$ in an arc $\gamma_{i} \subset \Gamma_{a} \cup \Gamma_{b}$ by $z^{(i, m)}(m \in \mathbb{N})$, so that for $m<n$ in a fundamental domain $D$ in the universal cover $\mathbb{R}^{2}$

- $\pi_{x}\left(\tilde{z}^{(i, m)}\right)<\pi_{x}\left(\tilde{z}^{(i, n)}\right) ; \quad z^{(i, m)}, z^{(i, n)} \in \gamma_{i} \subset \boldsymbol{a}, 0 \leq i \leq p_{1}-1$.

- $\pi_{x}\left(\tilde{z}^{(i, m)}\right)<\pi_{x}\left(\tilde{z}^{(i, n)}\right) ; \quad z^{(i, m)}, z^{(i, n)} \in \gamma_{i} \subset \boldsymbol{a}_{\mathbf{1}}, p_{1} \leq i \leq p_{1}+p_{2}-1$.

- $\pi_{y}\left(\tilde{z}^{(i, m)}\right)<\pi_{y}\left(\tilde{z}^{(i, n)}\right) ; \quad z^{(i, m)}, z^{(i, n)} \in \gamma_{i} \subset \boldsymbol{b}, p_{1} \leq i \leq p_{1}+p_{2}-1$.

Definition 4.2.10 (Main definition). Let $\mathcal{O} \subset \mathbb{T}^{2}$ be a period-n periodic orbit with rotation vector $v=\frac{k}{n}\left(p_{1}, p_{2}\right)$ such that $n \geq k\left(p_{1}+2 p_{2}\right)$ for $n, p_{1}, p_{2}$ relatively primes. Let $\gamma$ be a $\left(p_{1}, p_{2}\right)$ curve. Suppose that $\mathcal{O} \subset \gamma$, we will say that $(\mathcal{O}, \gamma)$ is a compatible orbit-curve pair, if they satisfy the following conditions:

(i) For each $\gamma_{i} \subset \Gamma_{a}$, we have that $\gamma_{i} \cap \Sigma_{a_{4}}=\left\{a_{4}^{(i, 1)}, \ldots, a_{4}^{(i, k)}\right\}$ where $\pi_{x}\left(\tilde{a}_{4}^{(i, 1)}\right)<\cdots<\pi_{x}\left(\tilde{a}_{4}^{(i, k)}\right)$ in $D$.

(ii) For each $\gamma_{i} \subset \Gamma_{b}$, we have that $\gamma_{i} \cap \Sigma_{b_{2}}=\left\{b_{2}^{(i, 1)}, \ldots, b_{2}^{(i, k)}\right\}$ where $\pi_{y}\left(\tilde{b}_{2}^{(i, 1)}\right)<\cdots<\pi_{y}\left(\tilde{b}_{2}^{(i, k)}\right)$ in $D$.

(iii) For each $\gamma_{i} \subset \Gamma_{b}$, we have that $\gamma_{i} \cap \Sigma_{a_{1}}=\left\{a_{1}^{(i, 1)}, \ldots, a_{1}^{(i, k)}\right\}$ where $\pi_{x}\left(\tilde{a}_{1}^{(i, 1)}\right)<\cdots<\pi_{x}\left(\tilde{a}_{1}^{(i, k)}\right)$ in $D$.

(iv) The remaining $\ell=n-k \cdot\left(p_{1}+2 p_{2}\right)$ points are distributed between $\Gamma_{a} \cap \boldsymbol{a}_{\mathbf{3}}$ and $\Gamma_{b} \cap \boldsymbol{b}_{\mathbf{1}}$ (preserving the order in D).

(v) Single outs those $f$-periodic orbits $\mathcal{O}$ which are contained in $\left(p_{1}, p_{2}\right)$-curve $\gamma$ such that the action of $f$ on $\mathcal{O}$ is compatible with a $2 \pi k / n$ rotation of $\gamma$. This is, there exists $\theta: \mathbb{S}^{1} \longrightarrow \mathbb{S}^{1}$ which is conjugated to a $2 \pi k / n$ rotation that extends $\left.\gamma^{-1} \circ f \circ \gamma\right|_{\gamma^{-1}(\mathcal{O})}$

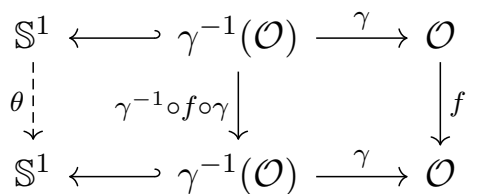

REMARK. The following picture is a caricature of a compatible pair $(\mathcal{O}, \gamma)$ for $p_{1}>p_{2}$. This image is a little far from the real situation because, by definition each orbit point is located in a different leaf in the fibered subsurface.






\section{REMARK.}

1. The 4.2.10(v) condition allows us to choose a very specific type of periodic orbits, once the orbit points are well distributed between the $p_{1}+p_{2}$ arcs in the circular version of $\gamma$, where them are spaced $2 \pi / n$, intuitively the homeomorphism "push" the points in the direction induced in the circle, $2 k \pi / n$ positions. Done this, we can read off the sequence of symbols $b_{1}$ 's, $b_{2}$ 's, $a_{1}$ 's, $a_{3}$ 's, $a_{4}$ 's around of the $\left(p_{1}, p_{2}\right)$-curve under the action of a $2 k \pi / n$-rotation. Fixed a periodic orbit $\mathcal{O}$, from the symbolic representation it is possible to calculate the relative positions of the points horizontally and vertically along this periodic orbit. This allow us to recognize the precise location in the torus of the periodic orbit points. The complete symbolic description will be developed in Section 4.5.

2. When $n=k\left(p_{1}+2 p_{2}\right)$, we have rational rotation vectors $v=\frac{1}{q}\left(p_{1}, p_{2}\right)$, for $q=p_{1}+2 p_{2}$. This vectors are located in the hypotenuse of the rotation triangle of equation $2 y=1-x$, and them are realized by periodic orbits with points in $\boldsymbol{a}_{\mathbf{1}}, \boldsymbol{a}_{\mathbf{4}}, \boldsymbol{b}_{\mathbf{2}}$. In particular, the orbit has no points in $\boldsymbol{a}_{3}, \boldsymbol{b}_{\mathbf{1}}$, this is $\ell=0$.

3. Under the previous definition we have $\ell$ points (between $\boldsymbol{b}_{\mathbf{1}}, \boldsymbol{a}_{\mathbf{3}}$ ) distribuited in a set of $p=p_{1}+p_{2}$ places, in this way the number of orbits in $\mathcal{O}_{v}$ is given by the expression (combinatory with repetitions),

$$
\left(\begin{array}{c}
p \\
\ell
\end{array}\right)=\left(\begin{array}{c}
p+\ell-1 \\
\ell
\end{array}\right)=\frac{(p+\ell-1) !}{\ell !(p-1) !}
$$

Note that when $\ell=0$, we only have a unique periodic orbit, which correspond to the periodic orbit in the hypothenuse of the rotation set. In particular, we will show that it is a finite order periodic orbit.

Example 1. Suppose that we want a $f$-periodic orbit with rotation vector $v=\frac{1}{9}(3,2)$. Note first that $v$ belongs to the interior of the rotation set. According to our previous definition, we will begin by defining the oriented simple closed $(3,2)$-curve. Naturally the arcs of the curve are connecting $\gamma_{1} \longrightarrow \gamma_{3} \longrightarrow \gamma_{5} \longrightarrow \gamma_{2} \longrightarrow \gamma_{4} \rightarrow$, we use the symbol " $\rightarrow$ " to indicate the restart of the sequence (see figure 4.7). From definition, we have three points of the orbit in $\boldsymbol{a}_{\mathbf{4}}$, two points in $\boldsymbol{b}_{\mathbf{2}}$, and two points in $\boldsymbol{a}_{\mathbf{1}}$ as follow

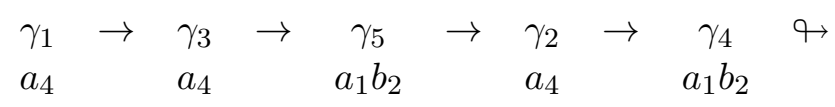


Figure 4.7: The picture on the left is circular version of the pair $(\mathcal{O}, \gamma)$. To the right we have the lift to the fundamental domain of the pair $(\mathcal{O}, \gamma)$. So far we have not considered points in the regions $\boldsymbol{a}_{\mathbf{3}}$ and $\boldsymbol{b}_{\mathbf{1}}$. 
Since $\operatorname{Per}(\mathcal{O})=9$, there are two points between $b_{1}, a_{3}$. Then, for this example we choose a point in $b_{1}$ and one in $a_{3}$ (we could also choose two in $b_{1}$, or two in $a_{3}$ ). By (iv), we can paste them in any arc between $\boldsymbol{a}$ or $\boldsymbol{b}$ as appropriate, the $b_{1}$ points can be pasted in $\gamma_{4}$ or $\gamma_{5}$, and the $a_{3}$ points in $\gamma_{1}$, or $\gamma_{2}$, or $\gamma_{3}$. We put them as follows

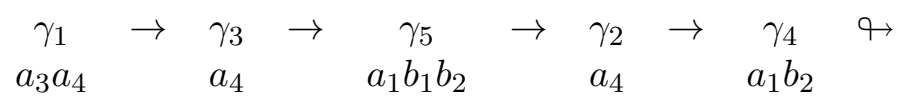

In this way, considering a $1 / 9$-rotation we can represent the periodic orbit as

$$
w=\left(a_{3} a_{4}^{2} a_{1} b_{1} b_{2} a_{4} a_{1} b_{2}\right)^{\infty} .
$$

To find the location of the orbit on the torus (see figure 4.8), we use the orders $\pi_{x}, \pi_{y}$ to know the relative positions of the points in the orbit (see equation 3.3).

$\begin{array}{ccccccccc}a_{3} & a_{4} & a_{4} & a_{1} & b_{1} & b_{2} & a_{4} & a_{1} & b_{2} \\ 3 & 6 & 4 & 1 & 1 & 3 & 5 & 2 & 2\end{array}$

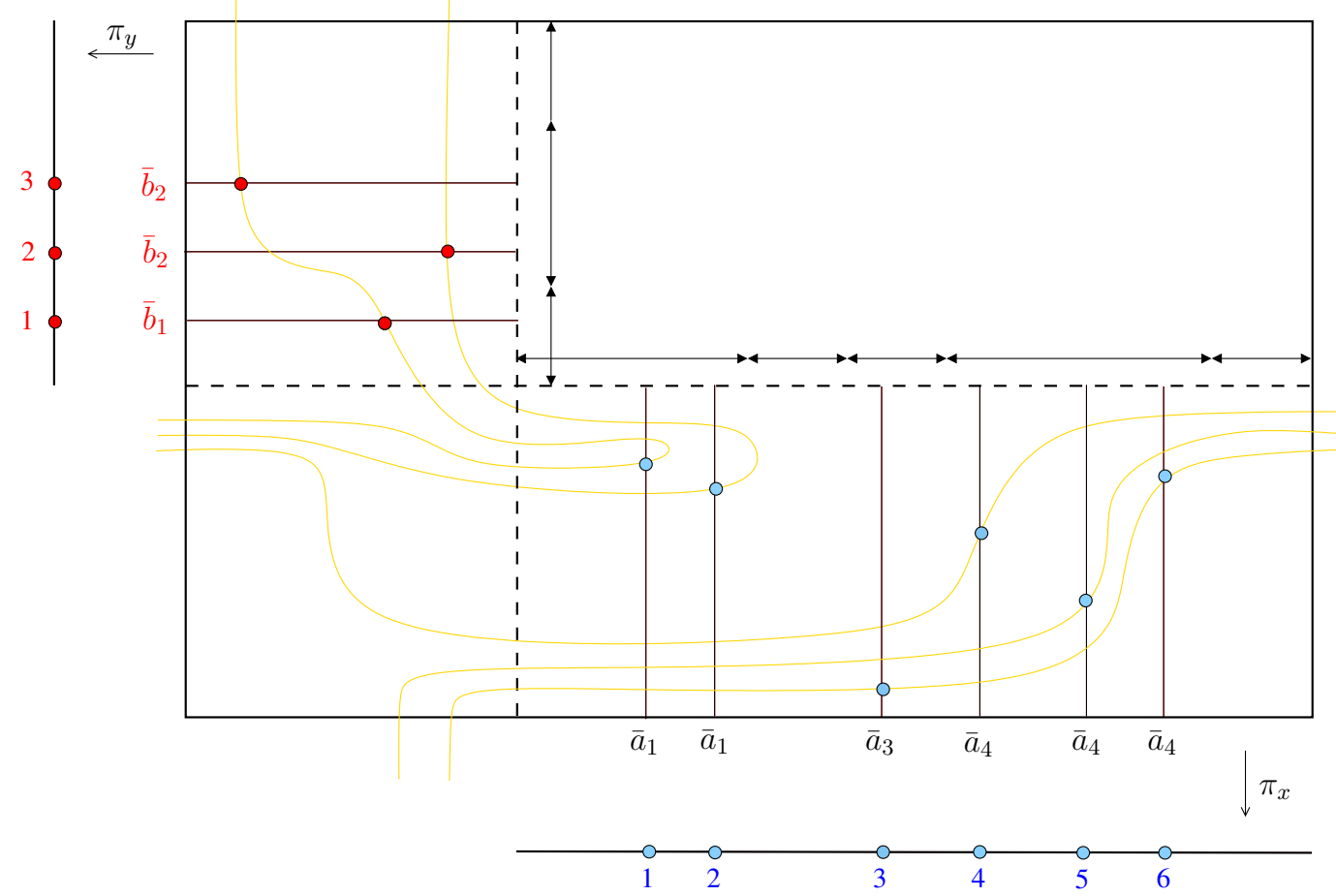

Figure 4.8: Lift to a fundamental domain of the pair $(\mathcal{O}, \gamma)$ with the horizontal projection for the points in $\boldsymbol{a}$-strip, and the vertical projection for the points in $\boldsymbol{b}$-strip.

Example 2. Consider the rotation vector $v=\frac{2}{9}(2,1)$. The simple closed curve $\gamma$ is connected as follow $\gamma_{1} \rightarrow \gamma_{2} \rightarrow \gamma_{3} \rightarrow$. From the Definition 4.2.10(i), we have four points in $\boldsymbol{a}_{4}$ distributed in the two simple arcs that running $\boldsymbol{a}$ as follow: $a_{4}^{(i, 1)}, a_{4}^{(i, 2)} \in \gamma_{i}, i=1,2$. By 4.2.10(ii)(iii) we have four points distributed in the arc $\gamma_{3}$, two points in $a_{1}$, and two in $b_{2}$. By 4.2.10(iv), a point $\left(\ell=n-k\left(p_{1}+2 p_{2}\right)=9-2(2+2 \cdot 1)\right)$ between $a_{3}, b_{1}$. Since there are three possible places to locate this element, we produce three different periodic orbits of rotation vector $v$ with curves in the class $(2,1)$. We illustrate this cases thinking in the circular version of the $(2,1)$-curves.

$$
w_{1}=\left(a_{4}^{2} a_{1} b_{1} b_{2} a_{4}^{2} a_{1} b_{2}\right)^{\infty} \quad w_{2}=\left(a_{4}^{2} a_{1} b_{2} a_{3} a_{4}^{2} a_{1} b_{2}\right)^{\infty} \quad w_{3}=\left(a_{4} a_{3} a_{4} a_{1} b_{1} b_{2} a_{4}^{2} a_{1} b_{2}\right)^{\infty}
$$



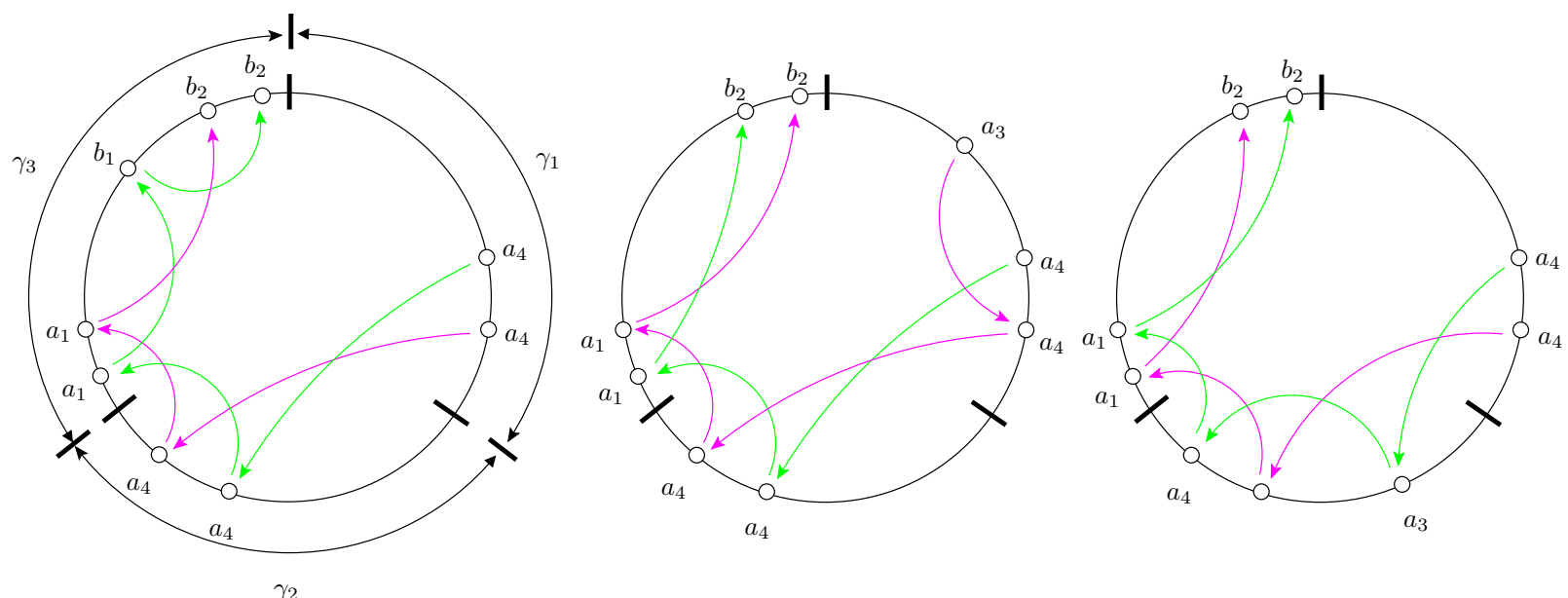

Figure 4.9: Dynamic on the circle of three different periodic orbits with rotation vector $v=\frac{2}{9}(2,1)$.

\subsection{Unfoldable and unimodal orbit-curve pair}

Let $\Omega$ be the set of pairs $(\mathcal{O}, \gamma)$ in the Definition 4.2.10. In this section, through the relative positions of the points in $\mathcal{O}$, we will consider orbits for which the $p_{1}$ arcs that pass through the regions $\boldsymbol{a}_{\mathbf{3}}, \boldsymbol{a}_{\mathbf{4}}$ in $\boldsymbol{a}$-strip, and the $p_{2}$ arcs that pass through $\boldsymbol{b}_{1}, \boldsymbol{b}_{\mathbf{2}}$ in the $b$-strip, their points not force folds in these regions, up to isotopy relative to the orbit. This $(\mathcal{O}, \gamma)$ pairs will be call unfoldable, in the next section we will show that this property allows us to pass the $p_{1}+p_{2}$ arcs monotonously in this regions (see Theorem 4.4.3). We will prove that this subset of $\Omega$ correspond to the set of pairs with finite order periodic orbits, $\Lambda$.

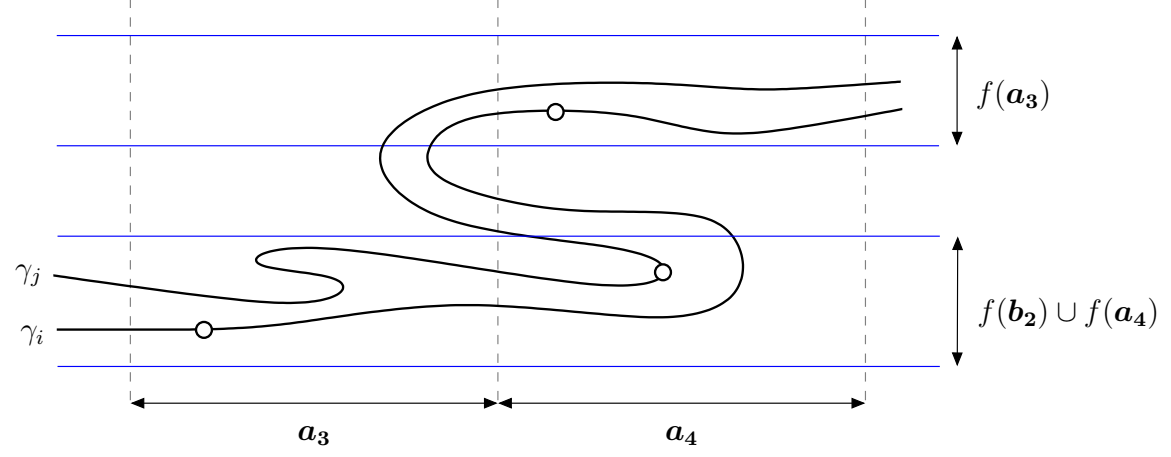

Figure 4.10: Fold between the arcs $\gamma_{i}, \gamma_{j}$ with $j>i$.

\section{Convention.}

- Let $\gamma_{i}$ be an $a$-arc $\left(0 \leq i \leq p_{1}-2\right)$ such that it contains at least an $a_{3}$ point. We call

$$
S_{i}=\bigcup_{j=i}^{p_{1}-1}\left[\gamma_{j} \cap\left(\Sigma_{a_{3}} \cup \Sigma_{a_{4}}\right)\right] \subset f\left(\boldsymbol{b}_{\mathbf{2}} \cup \boldsymbol{a}_{\mathbf{4}}\right)
$$

to the set of $a_{3}$ 's, $a_{4}$ 's in $f\left(\boldsymbol{b}_{\mathbf{2}}\right) \cup f\left(\boldsymbol{a}_{\mathbf{4}}\right)$, which belong to the arcs with index greater or equal than $i$. For $\gamma_{i}$ fixed, we define $E_{i}=\max _{s \in S_{i}}\left\{\pi_{x}(\tilde{s}) \mid \tilde{s} \in D\right\}$, and $F_{i}=\pi_{x}\left(\tilde{f}\left(\tilde{a}_{3}^{(i, 1)}\right)\right)$.

- Let $\gamma_{i}$ be a $b$-arc $\left(p_{1} \leq i \leq p_{1}+p_{2}-2\right)$ such that it contains at least a $b_{1}$ point. We call 


$$
T_{i}=\bigcup_{j=i}^{p_{1}+p_{2}-1}\left[\gamma_{j} \cap\left(\Sigma_{b_{1}} \cup \Sigma_{b_{2}}\right)\right] \subset f\left(\boldsymbol{a}_{\mathbf{1}}\right)
$$

to the set of $b_{1}$ 's, $b_{2}$ 's in $f\left(\boldsymbol{a}_{1}\right)$, which belong to the arcs with index greater or equal than $i$. For $\gamma_{i}$ fixed, we define $L_{i}=\max _{t \in T_{i}}\left\{\pi_{y}(\tilde{t}) \mid \tilde{t} \in D\right\}$, and $M_{i}=\pi_{y}\left(\tilde{f}\left(\tilde{b}_{1}^{(i, 1)}\right)\right)$.

Definition 4.3.1. Let $(\mathcal{O}, \gamma)$ be a compatible orbit-curve pair. We will say that the pair $(\mathcal{O}, \gamma)$ is unfoldable, if in the following two groups $(F 1)-(F 3),(G 1)-(G 3)$, one of the conditions is true:

(F1) There are no a $a_{3}$ 's points in $\boldsymbol{a}$.

(F2) Only the arc $\gamma_{p_{1}-1}$ in a has $a_{3}$ 's points.

(F3) For each $\gamma_{i} \subset \Gamma_{a} \backslash \gamma_{p_{1}-1}$ with at least an $a_{3}$ 's point; we have $E_{i}<F_{i}$.

(G1) There are no $b_{1}$ 's points in $\boldsymbol{b}$.

(G2) Only the arc $\gamma_{p_{1}+p_{2}-1}$ in $\boldsymbol{b}$ has $b_{1}$ 's points.

(G3) For each $\gamma_{i} \subset \Gamma_{b} \backslash \gamma_{p_{1}+p_{2}-1}$ with at least a $b_{1}$ 's point; we have $L_{i}<M_{i}$.

REMARK. The $F 1$ and $G 1$ cases occurs together when a periodic orbit has no points either $\boldsymbol{a}_{\mathbf{3}}$ or $\boldsymbol{b}_{\mathbf{1}}$, so this periodic orbit realizes a vector $v=\frac{1}{q}\left(p_{1}, p_{2}\right)$ in the hypotenuse of the rotation set.

Example 3. Let $v=\frac{2}{17}(3,2)$ be a rotation vector for a lift $\tilde{f}$ from $f$. We describe the dynamics of two pairs $(\mathcal{O}, \gamma),\left(\mathcal{O}^{\prime}, \gamma^{\prime}\right)$ with rotation vector $v$ such that the first of them will be unfoldable. Consider the following symbolic descriptions:

$$
\begin{aligned}
& w=\left(a_{1} b_{1} b_{2} a_{4} a_{3} a_{4} a_{1} b_{2} a_{4} a_{1} b_{1} b_{2} a_{4}^{2} a_{1} b_{2} a_{4}\right)^{\infty} \\
& w^{\prime}=\left(a_{1} b_{1} b_{2} a_{3} a_{4}^{2} a_{1} b_{2} a_{4} a_{1} b_{1} b_{2} a_{4}^{2} a_{1} b_{2} a_{4}\right)^{\infty} .
\end{aligned}
$$

In the first case, once the word $w$ is ordered, we sketch the pair $(\mathcal{O}, \gamma)$. This design is a simplified version of the real picture, here the the orbit points have been moved in the leafs that contain them.

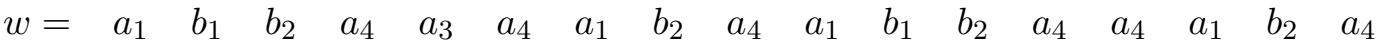

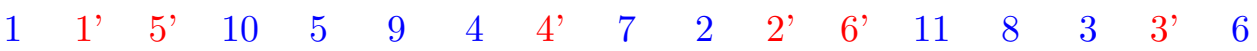




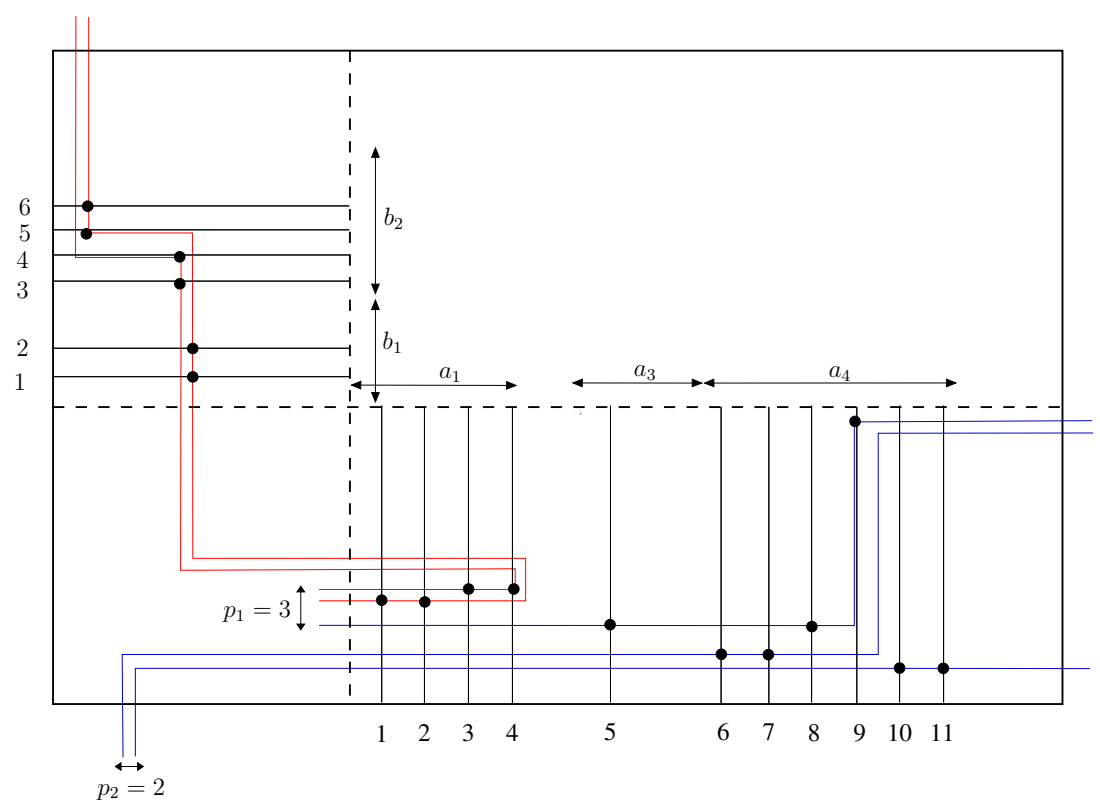

Figure 4.11: The pair $(\mathcal{O}, \gamma)$ with symbolic description $w$ is unfoldable.

According to the definition the first pair $(\mathcal{O}, \gamma)$ is unfoldable because this satisfy (F2) and (G3), here $L_{1}=\max \{3,4\}$ and $M_{1}=5$, then $L_{1}<M_{1}$. The second pair $\left(\mathcal{O}^{\prime}, \gamma^{\prime}\right)$ can not be unfolded, the arcs in the $\boldsymbol{b}$-strip do not satisfy the conditions from (G1) to (G3) (note in (G3) that $L_{1}=\max \{4,5\}$ and $M_{1}=3$, then $L_{1}>M_{1}$.), although the arcs in the $\boldsymbol{a}$ strip do comply with the property (F3); $E_{1}=\max \{6,7,8,9\}, F_{1}=11$ with $E_{1}<F_{1}$.

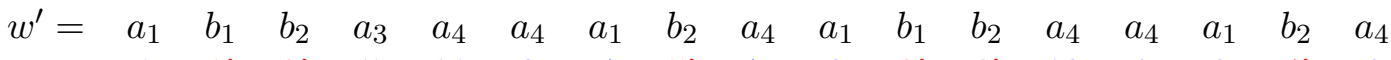

$$
\begin{aligned}
& \begin{array}{lllllllllllllllll}
1 & 1 & 3 & 5 & 11 & 9 & 4 & 5 & 7 & 2 & 2 & 6 & 10 & 8 & 3 & 4 & 6
\end{array}
\end{aligned}
$$

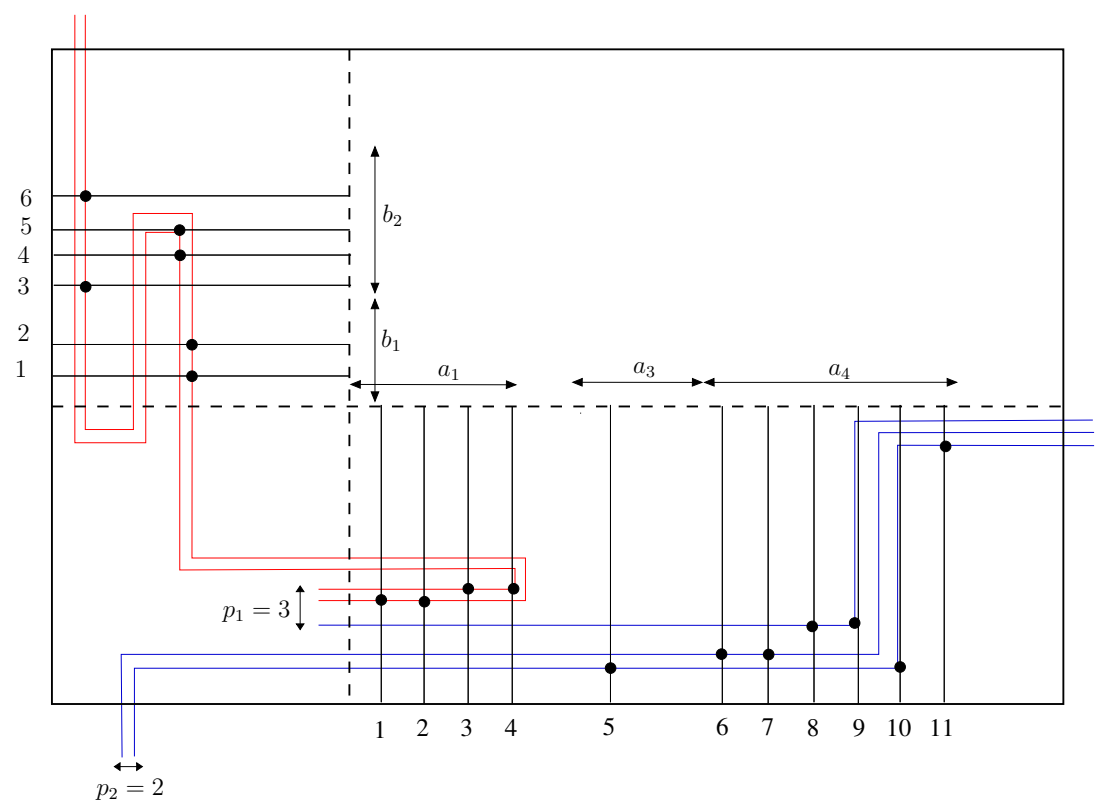

Figure 4.12: The pair $\left(\mathcal{O}^{\prime}, \gamma^{\prime}\right)$ with symbolic description $w^{\prime}$ can not be unfolded.

REMARK. The "unfoldable" condition is related to the points of the orbit, and we may use it to describe how the curve passes through some points. In the second pair of the previous example, we 
can see that the curve defines a fold on the $\boldsymbol{b}$-strip, which cannot be deformed up to isotopy (notice that the curve may also go through the points without making a "fold"). These type of orbits will be not considered, we will prove that the orbits that satisfy the statements in the Definition 4.3.1, making isotopy relative to the orbit, the folds can be undone. We call unimodal to this property (see Theorem 4.4.3). The unfoldable property will be a necessary condition for obtaining fopos (see Theorem 4.4.8).

\section{Convention.}

- For us a monotone arc means that given a lift to the universal cover of a arc $\gamma_{i},(0 \leq i \leq$ $\left.p_{1}+p_{2}-1\right)$ passing through of the horizontal band $\boldsymbol{a}$ from $\boldsymbol{a}_{\mathbf{1}}$ to $\boldsymbol{a}_{\mathbf{5}}$, or vertical band $\boldsymbol{b}$ from $\boldsymbol{b}_{\mathbf{1}}$ to $\boldsymbol{b}_{\mathbf{3}}$, the projections on the first or second factor, $\pi_{x}, \pi_{y}$ restricted to the lift arc is injective.

- We will use the term unimodal arc to indicate that the arc tours $\boldsymbol{a}_{\mathbf{1}}$ round-trip without additional folds.

Definition 4.3.2. Let $(\mathcal{O}, \gamma)$ be a compatible orbit-curve pair. We will say that the pair $(\mathcal{O}, \gamma)$ is unimodal, if up to homotopy relative to the orbit the curve looks like the figure (4.13), this is

(i) The $p_{1}$ arcs that go through the horizontal band from $\boldsymbol{a}_{\mathbf{1}}$ to $\boldsymbol{a}_{\mathbf{5}}$ are a-monotones.

(ii) The $p_{2}$ arcs that go through the vertical band from $\boldsymbol{b}_{\mathbf{1}}$ to $\boldsymbol{b}_{\mathbf{3}}$ are b-monotones.

(iii) The $p_{2}$ arcs on the top of the horizontal band are unimodal on $\boldsymbol{a}_{\mathbf{1}}$.

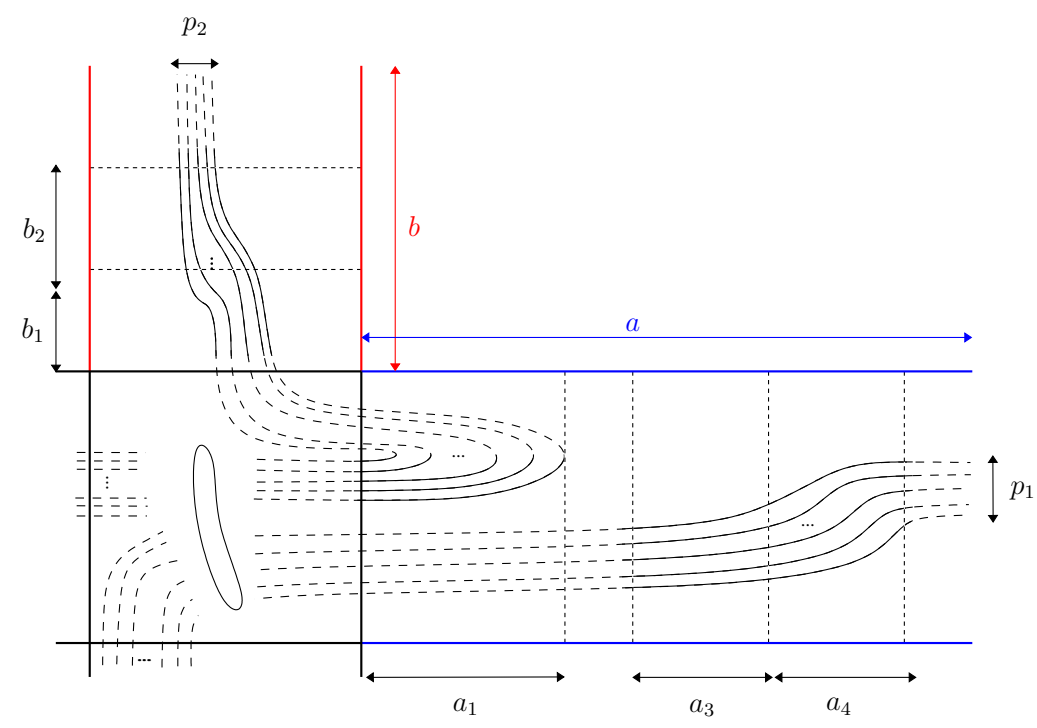

Figure 4.13: These lines are joined here in the only possible way to make a simple closed curve.

REMARK. Every pair $(\mathcal{O}, \gamma)$ with periodic orbit of rotation vector $v=\frac{k}{n}(1,1), n \geq 3 k$ satisfies trivially the condition of being unimodal.

Example 4. Consider the example 3 from the Section 4.3. Observe that the curve in the pair $(\mathcal{O}, \gamma)$ can be deformed isotopically satisfying the conditions from (i)-(iii) in the unimodal definition (see figure 4.11). The curve in the second pair $\left(\mathcal{O}^{\prime}, \gamma^{\prime}\right)$ may or may not be represented as a unimodal curve. In particular, the Figure 4.12 illustrate the case in which the curve is not unimodal (we choose the $\left(p_{1}, p_{2}\right)$-curve which is more likely to be isotopic to its image relative to the periodic orbit). These cases will be left out through the theorem 4.4.3. 


\subsection{Main theorems and proofs}

Convention. From now on, we will consider the case $p_{1}>p_{2}$ (which means that the number of $a$-arcs is greater than the number of $b$-arcs). The propositions and theorems in this sections can be extended to the case $p_{1}<p_{2}$. In particular, if $p_{1}=p_{2}=1$, the main Theorem 4.4.8 follows directly.

Remark. From the Definitions 3.1.1, 4.2.10, we have for $p_{1}>p_{2}$

$$
\begin{array}{lll}
k p_{1} & \text { points in } & \Sigma_{a_{4}} \subset \boldsymbol{a}_{\mathbf{4}}^{\circ} ; \\
k p_{2} & \text { points in } & \Sigma_{b_{2}} \subset \boldsymbol{b}_{\mathbf{2}}^{\circ} ; \\
k p_{2} & \text { points in } & \Sigma_{a_{1}} \subset \boldsymbol{a}_{\mathbf{1}}^{\circ} ; \\
\ell_{a} & \text { points in } & \Sigma_{a_{3}} \subset \boldsymbol{a}_{\mathbf{3}}^{\circ} ; \\
\ell_{b} & \text { points in } & \Sigma_{b_{1}} \subset \boldsymbol{b}_{\mathbf{1}}{ }^{\circ} ;
\end{array}
$$

distributed as follows:

$\begin{array}{lll}k\left(p_{2}-p_{1}\right) & \text { points in } & \Sigma_{a_{4}} \cap\left(\cup_{i=0}^{p_{1}-p_{2}-1} \gamma_{i}\right) ; \\ k p_{2} & \text { points in } & \Sigma_{a_{4}} \cap\left(\cup_{i=p_{1}-p_{2}}^{p_{1}+p_{2}-1} \gamma_{i}\right) ; \\ k p_{2} & \text { points in } & \Sigma_{b_{2}} \cap \Gamma_{b} ; \\ k p_{2} & \text { points in } & \Sigma_{a_{1}} \cap \Gamma_{b} ; \\ \ell_{a} & \text { points in } & \Sigma_{a_{3}} \cap \Gamma_{a} ; \\ \ell_{b} & \text { points in } & \Sigma_{b_{1}} \cap \Gamma_{b} .\end{array}$

Proposition 4.4.1. Let $(\mathcal{O}, \gamma)$ be a orbit-curve pair with rotation vector $v=\frac{k}{n}\left(p_{1}, p_{2}\right)$ with $n \geq$ $k\left(p_{1}+2 p_{2}\right)$ for $p_{1}>p_{2}$. Then

(i) $f\left(\Sigma_{a_{1}} \cup \Sigma_{b_{1}}\right)=\Sigma_{b_{1}} \cup \Sigma_{b_{2}}$;

(ii) $f\left(\Sigma_{b_{2}}\right) \cup f\left(\Sigma_{a_{3}}\right) \cup f\left(\Sigma_{a_{4}} \cap\left(\cup_{i=0}^{p_{1}-p_{2}-1} \gamma_{i}\right)\right)=\Sigma_{a_{3}} \cup \Sigma_{a_{4}}$;

(iii) $f\left(\Sigma_{a_{4}} \cap\left(\cup_{i=p_{2}}^{p_{1}-1} \gamma_{i}\right)\right)=\Sigma_{a_{1}}$.

Proof. (i) Let $p_{1}>p_{2}$. By $4.2 .10\left(\right.$ ii)(iv), there exist $k p_{2}$ points in $\boldsymbol{a}_{\mathbf{1}}$ and $\ell_{b}$ in $\boldsymbol{b}_{\mathbf{1}}$. From the Definition 3.1.1, $f\left(\Sigma_{a_{1}}\right) \subset \Sigma_{b_{1}} \cup \Sigma_{b_{2}}$ and $f\left(\Sigma_{b_{1}}\right) \subset \Sigma_{b_{1}} \cup \Sigma_{b_{2}}$. Then

$$
f\left(\Sigma_{a_{1}} \cup \Sigma_{b_{1}}\right)=f\left(\Sigma_{a_{1}}\right) \cup f\left(\Sigma_{b_{1}}\right) \subset \Sigma_{b_{1}} \cup \Sigma_{b_{2}},
$$

and the last contains exactly $k p_{2}+\ell_{b}$ points. Since $\Sigma_{a_{1}} \cap \Sigma_{b_{1}}=$ and $f$ is injective, it follows that $f\left(\Sigma_{a_{1}} \cup \Sigma_{b_{1}}\right)=\Sigma_{b_{1}} \cup \Sigma_{b_{2}}$.

(ii) Let $p_{1}>p_{2}$. By 4.2.10(i)(ii)(iv), the regions $\boldsymbol{a}_{\mathbf{4}}, \boldsymbol{b}_{\mathbf{2}}$ and $\boldsymbol{a}_{\mathbf{3}}$ contains $k p_{1}, k p_{2}$ and $\ell_{a}$ points respectively. Then, $f\left(\Sigma_{b_{2}} \cup \Sigma_{a_{3}} \cup\left[\Sigma_{a_{4}} \cap\left(\cup_{i=0}^{p_{1}-p_{2}-1} \gamma_{i}\right)\right]\right)$ has $k p_{2}+\ell_{a}+k\left(p_{1}-p_{2}\right)=\ell_{a}+k p_{1}$ points. From the Definiton 3.1.1, $f\left(\Sigma_{b_{2}}\right) \subset \Sigma_{a_{3}} \cup \Sigma_{a_{4}}, f\left(\Sigma_{a_{3}}\right) \subset \Sigma_{a_{3}} \cup \Sigma_{a_{4}}$ and $f\left(\Sigma_{a_{4}} \cap \cup_{i=0}^{p_{1}-p_{2}-1} \gamma_{i}\right)$. Thus,

$$
f\left(\Sigma_{b_{2}} \cup \Sigma_{a_{3}} \cup\left[\Sigma_{a_{4}} \cap\left(\cup_{i=0}^{p_{1}-p_{2}-1} \gamma_{i}\right)\right]\right) \subset \Sigma_{a_{3}} \cup \Sigma_{a_{4}} .
$$

We claim that the above inclusion is really an equality. Suppose that

$$
\Sigma_{a_{3}} \cup \Sigma_{a_{4}} \nsubseteq f\left(\Sigma_{b_{2}} \cup \Sigma_{a_{3}} \cup\left[\Sigma_{a_{4}} \cap\left(\cup_{i=0}^{p_{1}-p_{2}-1} \gamma_{i}\right)\right]\right) .
$$

Then, it must exist a orbit point in $\Sigma_{a_{3}} \cup \Sigma_{a_{4}}$ which has not image of a point in $\Sigma_{b_{2}} \cup \Sigma_{a_{3}} \cup$ $\left[\Sigma_{a_{4}} \cap\left(\cup_{i=0}^{p_{1}-p_{2}-1} \gamma_{i}\right)\right]$, but this is not possible since from Definition 3.1.1,

$$
f\left(\Sigma_{b_{2}} \cup \Sigma_{a_{3}} \cup\left[\Sigma_{a_{4}} \cap\left(\cup_{i=0}^{p_{1}-p_{2}-1} \gamma_{i}\right)\right]\right) \cap\left(\Sigma_{a_{3}} \cup \Sigma_{a_{4}}\right)=\emptyset .
$$


(iii) The proof is similar to the previous ones.

Notation. Call $\ell_{a}^{i}$ to the net number of $a_{3}$ 's in an $a$-arc $\gamma_{i}\left(0 \leq i \leq p_{1}-1\right)$, and $\ell_{b}^{i}$ to the net number of $b_{1}$ 's in a $b$-arc $\gamma_{i}\left(p_{1} \leq i \leq p_{1}+p_{2}-1\right)$.

Proposition 4.4.2. Let $(\mathcal{O}, \gamma)$ be a orbit-curve pair with rotation vector $v=\frac{k}{n}\left(p_{1}, p_{2}\right)$ with $n \geq$ $k\left(p_{1}+2 p_{2}\right)$ for $p_{1}>p_{2}$, and let $\delta=\left(i+p_{2}\right) \bmod \left(p_{1}+p_{2}\right)$. Then

(i) $f\left(\gamma_{i} \cap\left(\Sigma_{a_{1}} \cup \Sigma_{b_{1}}\right)\right)=\gamma_{i} \cap\left(\Sigma_{b_{1}} \cup \Sigma_{b_{2}}\right) ; i=p_{1}, \ldots, p_{1}+p_{2}-1$.

(ii) $f\left(\gamma_{i} \cap \Sigma_{b_{2}}\right) \subset \gamma_{\delta} \cap\left(\Sigma_{a_{3}} \cup \Sigma_{a_{4}}\right) ; i=p_{1}, \ldots, p_{1}+p_{2}-1, \delta=0, \ldots, p_{2}-1$.

(iii) $f\left(\gamma_{i} \cap \Sigma_{a_{3}}\right) \subset \gamma_{i} \cap\left(\Sigma_{a_{3}} \cup \Sigma_{a_{4}}\right) ; i=0, \ldots, p_{1}-1$.

(iv) $f\left(\gamma_{i} \cap \Sigma_{a_{4}}\right) \subset \gamma_{\delta} \cap\left(\Sigma_{a_{3}} \cup \Sigma_{a_{4}}\right) ; i=0, \ldots, p_{1}-p_{2}-1, \delta=p_{2}, \ldots, p_{1}-1$,

$f\left(\gamma_{i} \cap \Sigma_{a_{4}}\right)=\gamma_{\delta} \cap \Sigma_{a_{1}} ; \quad i=p_{1}-p_{2}, \ldots, p_{1}-1, \delta=p_{1}, \ldots, p_{1}+p_{2}-1$.

Proof. (i) By Propositions 4.2.6, 4.2.5 and Corollary 4.2.7 the rectangular regions $f\left(\boldsymbol{a}_{\mathbf{1}}\right), f\left(\boldsymbol{b}_{\mathbf{1}}\right)$ are contained in $\boldsymbol{b}$-strip with $f\left(\partial^{\text {in }}\left(\boldsymbol{a}_{\mathbf{1}}\right)\right) \subset \partial^{\text {out }}(\boldsymbol{b})$ and $f\left(\partial^{\text {out }}\left(\boldsymbol{b}_{\mathbf{1}}\right)\right) \subset \partial^{\text {out }}(\boldsymbol{b})$ respectively. From 4.4.1(i), $f\left(\Gamma_{b} \cap\left(\Sigma_{a_{1}} \cup \Sigma_{b_{1}}\right)\right)=\Gamma_{b} \cap\left(\Sigma_{b_{1}} \cup \Sigma_{b_{2}}\right)$. Let $\gamma_{i} \subset \Gamma_{b}$, by 4.2.10(i)(iii)(iv) $\gamma_{i}$ contain the following sets of ordered points: $\gamma_{i} \cap \Sigma_{a_{1}}=\left\{a_{1}^{(i, 1)}, \ldots, a_{1}^{(i, k)}\right\}, \gamma_{i} \cap \Sigma_{b_{1}}=\left\{b_{1}^{(i, 1)}, \ldots, b_{1}^{\left(i, \ell_{b}^{i}\right)}\right\}\left(\ell_{b}^{i} \geq 0\right)$ and $\gamma_{i} \cap \Sigma_{b_{2}}=\left\{b_{2}^{(i, 1)}, \ldots, b_{2}^{(i, k)}\right\}$. By 4.2.10(v), the homeomorphism $f$ "push" one by one the points in $\gamma_{i}$ as follows: the $k$ points in $\gamma_{i} \cap \Sigma_{a_{1}}$ are carried in the first $k$ points in $\gamma_{i} \cap\left(\Sigma_{b_{1}} \cup \Sigma_{b_{2}}\right)$ from $\ell_{b}^{i}+k$, the rest of the points are image of the $\ell_{b}^{i}$ points in $\gamma_{i} \cap \Sigma_{b_{1}}$. In particular, if $\ell_{b}^{i}=0$ then $f\left(a_{1}^{(i, 1)}\right)=b_{2}^{(i, 1)}, \ldots, f\left(a_{1}^{(i, k)}\right)=b_{2}^{(i, k)}$.

(ii) From the Proposition 4.4.1(ii), $f\left(\Gamma_{b} \cap \Sigma_{b_{2}}\right) \subset \Gamma_{a} \cap\left(\Sigma_{a_{3}} \cup \Sigma_{a_{4}}\right)$. Let $\gamma_{\delta} \subset \Gamma_{a}$. By Definition $4.2 .9, \gamma_{\delta}$ is a continuation of $\gamma_{i}$ with $\delta=\left(i+p_{2}\right) \bmod \left(p_{1}+p_{2}\right)$, and by 4.2.10(i)(ii)(iv), $\gamma_{i} \cap \Sigma_{b_{2}}=$ $\left\{b_{2}^{(i, 1)}, \ldots, b_{2}^{(i, k)}\right\}$ and $\gamma_{\delta} \cap\left(\Sigma_{a_{3}} \cup \Sigma_{a_{4}}\right)=\left\{a_{3}^{(\delta, 1)}, \ldots, a_{3}^{\left(\delta, \ell_{a}^{\delta}\right)}, a_{4}^{(\delta, 1)}, \ldots, a_{4}^{(\delta, k)}\right\}\left(\ell_{a}^{\delta} \geq 0\right)$. It follows from 4.2.10(v) that $f$ carries the $k$ points in $\gamma_{i} \cap \Sigma_{b_{2}}\left(p_{1} \leq i \leq p_{1}+p_{2}-1\right)$ to the first $k$ points in $\Sigma_{a_{3}} \cup \Sigma_{a_{4}}$, from $k+\ell_{a}^{\delta}$. The $\ell_{a}^{\delta}$ points in $\gamma_{i} \cap \Sigma_{a_{3}}$ are also carried at the $\ell_{a}^{\delta}$ points in the queue of $\gamma_{\delta} \cap\left(\Sigma_{a_{3}} \cup \Sigma_{a_{4}}\right)$.

(iii)-(iv) The proof is similar to the previous ones.

REMARK. For $0<\ell_{a}^{\delta} \leq k$ in 4.4.2(iv), we see that:

$$
\begin{array}{lll}
\left\{a_{4}^{(i, 1)}, \ldots, a_{4}^{(i, 1)}\right\} & \subset \gamma_{i} \cap \Sigma_{a_{4}} & ; i=0, \ldots, p_{1}-p_{2}-1, \\
\left\{a_{3}^{(\delta, 1)}, \ldots, a_{3}^{\left(\delta, \ell_{a}^{\delta}\right)}, a_{4}^{(\delta, 1)}, \ldots, a_{4}^{(\delta, k)}\right\} & \subset \gamma_{\delta} \cap\left(\Sigma_{a_{3}} \cup \Sigma_{a_{4}}\right) & ; \delta=p_{2}, \ldots, p_{1}-1,
\end{array}
$$

where

$$
f\left(a_{4}^{(i, 1)}\right)=a_{3}^{(\delta, 1)}, \ldots, f\left(a_{4}^{\left(i, \ell_{a}^{\delta}\right)}\right)=a_{3}^{\left(\delta, \ell_{a}^{\delta}\right)}, f\left(a_{4}^{\left(i, \ell_{a}^{\delta}+1\right)}\right)=a_{4}^{(\delta, 1)}, \ldots, f\left(a_{4}^{(i, k)}\right)=a_{4}^{\left(k-\ell_{a}^{\delta}\right)},
$$

and

$$
f\left(a_{3}^{(\delta, 1)}\right)=a_{4}^{\left(\delta, k-\ell_{a}^{\delta}+1\right)}, \ldots, f\left(a_{3}^{\left(i, \ell_{a}^{\delta}\right)}\right)=a_{4}^{(\delta, k)}
$$

(see figure 4.14). 


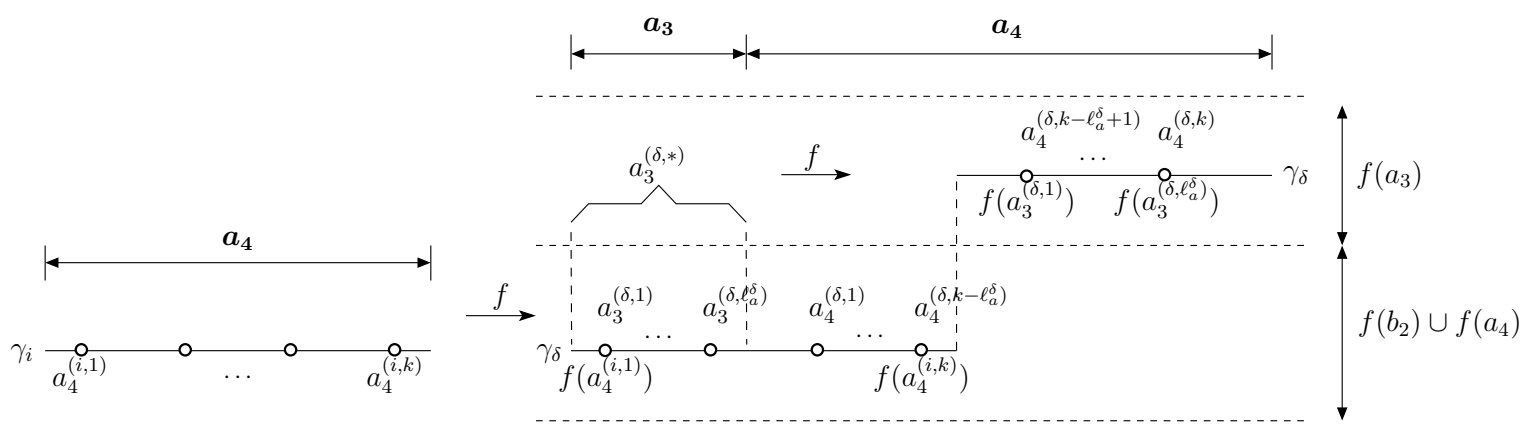

Figure 4.14: Behavior of the $a_{4}$ 's points in an a-arc, which have image in the arc $\gamma_{\delta}$ on the $\boldsymbol{a}$-strip by $f$. In this case the $\gamma_{\delta}$ arc contains $0<\ell_{a}^{\delta} \leq k$ points in $\boldsymbol{a}_{\mathbf{3}}$.

Convention. We will use the notation $E_{i}, F_{i}, L_{i}, M_{i}$ in the Definition 4.3 .1 to refer us to the leafs where the values come from. Thus, we can write $L_{i}<M_{i}$ to indicate that the leaf $L_{i}$ is down the leaf $M_{i}$, and write $E_{i}<F_{i}$ to indicate that the leaf $E_{i}$ is to left of leaf $F_{i}$.

Theorem 4.4.3. Let $(\mathcal{O}, \gamma)$ be a compatible orbit-curve pair. If the pair $(\mathcal{O}, \gamma)$ is unfoldable, then it is unimodal.

REMARK. The simple closed curve $\gamma$ has a representative $\eta$ in the isotopy class such that $(\mathcal{O}, \eta)$ is unimodal.

Proof. (Theorem 4.4.3) Suppose that the pair $(\mathcal{O}, \gamma)$ is unfoldable. Since that in the definition the $\boldsymbol{a}, \boldsymbol{b}$ strips satisfy the same group of conditions, it is enough prove a group of them. We assume that the arcs on $a$-strip are $a$-monotones. The proof will be divided into the three cases into $\boldsymbol{b}$-strip.

Case 1. Suppose that there are no $b_{1}$ 's in the $\boldsymbol{b}$-strip. By 4.2.10(ii), each $\gamma_{i}\left(p_{1} \leq i \leq p_{1}+p_{2}-1\right)$ passes through the $k$ points $\left\{b_{2}^{(i, 1)}, b_{2}^{(i, 2)}, \ldots, b_{2}^{(i, k)}\right\}=\gamma_{i} \cap \Sigma_{b_{2}} \subset b_{2}^{\circ}$ following the order induced by relation $\pi_{y}\left(\tilde{b}_{2}^{(i, 1)}\right)<\cdots<\pi_{y}\left(\tilde{b}_{2}^{(i, k)}\right)$ in a fundamental domain $D$ of the universal cover $\mathbb{R}^{2}$. The $k p_{2}$ points in the set $\cup_{i=p_{1}}^{p_{1}+p_{2}-1}\left(\gamma_{i} \cap \Sigma_{b_{2}}\right)$ are placed in different leaves $N_{1}, N_{2}, \ldots, N_{k p_{2}}$ in the interior of $f\left(\boldsymbol{a}_{\mathbf{1}}\right) \subset \boldsymbol{b}_{\mathbf{2}}$, and them are completely ordered in the following way $N_{1}<N_{2}<\cdots N_{k p_{2}}$.

Let $R=\boldsymbol{b}$. By 4.2.2, $R$ is a closed rectangular region. From Definition 4.2.9(ii), the set of $\Gamma_{b} \cap R$ arcs is a finite collection of cross-cuts in $R$. Since $\gamma_{p_{1}} \prec \cdots \prec \gamma_{p_{1}+p_{2}-1}$ in $R$, we can choose the points $s_{i j} \in \gamma_{i} \cap N_{j},\left(i=p_{1}, \ldots, p_{1}+p_{2}-1\right)\left(j=1, \ldots, k p_{2}\right)$ such that in a fundamental domain $D$ :

For $j$ fixed;

$$
\pi_{x}\left(\tilde{s}_{p_{1 j}}\right)<\pi_{x}\left(\tilde{s}_{\left(p_{1}+1\right) j}\right)<\cdots<\pi_{x}\left(\tilde{s}_{\left(p_{1}+p_{2}-1\right) j}\right)
$$

For $i$ fixed;

$$
\pi_{y}\left(\tilde{s}_{i 1}\right)<\pi_{y}\left(\tilde{s}_{i 2}\right)<\cdots<\pi_{x}\left(\tilde{s}_{i\left(k p_{2}\right)}\right) .
$$

Let $Q_{i}=\left\{s_{i 1}, s_{i 2}, \ldots, s_{i\left(k p_{2}\right)}\right\}$ and denote by $Q=\cup_{i=p_{1}}^{p_{+} p_{2}-1} Q_{i}$. In particular, $\gamma_{i} \cap \Sigma_{b_{2}} \subset Q_{i}$. Let $\eta_{i}$ be the piecewise geodesic curve that pass an orderly way through the points $Q_{i}$ (i.e., preserving the natural order of the leaves in $\boldsymbol{b}$-strip) with endpoints $s_{i 0}, s_{i\left(k p_{2}+1\right)} \in \gamma_{i} \cap \partial R$. We claim that $\eta_{i} \cap \eta_{i+1}=\emptyset\left(i=p_{1}, \ldots, p_{1}+p_{2}-2\right)$. This follows from equations (4.5)(4.6) since in any fundamental domain for $i, j$ fixed the line segments $\tilde{\eta}_{i j}=\pi^{-1}\left(\eta_{i j}\right), \tilde{\eta}_{(i+1) j}=\pi^{-1}\left(\eta_{(i+1) j}\right)$ are disjoints, where $\eta_{i j} \subset \eta_{i}, \eta_{(i+1) j} \subset \eta_{i+1}$ have endpoints $s_{i j}, s_{i(j+1)}$ and $s_{(i+1) j}, s_{(i+1)(j+1)}$ respectively.

Let $\eta$ be the oriented simple closed curve such that $\eta_{i} \subset \eta$ and outside of $\boldsymbol{b}$-strip is equal to $\gamma$. Note that $\eta$ is a $\left(p_{1}, p_{2}\right)$-curve such that $\eta \cap \gamma \supset Q$. We want to show that $\eta$ is homotopic to $\gamma$ relative to $Q$. Being $\eta$ equal to $\gamma$ outside $\boldsymbol{b}$-strip, we just need to see what happens in $\boldsymbol{b}$. Let $s_{i j} \in Q$ such that $\tilde{s}_{i j}$ is any point in the fiber of $\pi^{-1}\left(s_{i j}\right)$ in $\mathbb{R}^{2}$. From path lifting property (2.5.9), there exist unique lifts from $\eta, \gamma$ for which $\tilde{s}_{i j} \in \tilde{\gamma} \cap \tilde{\eta}$ as follows: we begin by lifting the geodesic 
$\operatorname{arcs} \gamma_{i j}, \eta_{i j}$ of endpoints $s_{i j}, s_{i(j+1)}$ to the $\operatorname{arcs} \tilde{\gamma}_{i j}, \tilde{\eta}_{i j}$ with initial point $\tilde{s}_{i j}$. Then, we apply the property again at the endpoint $\tilde{s}_{i(j+1)}=\pi^{-1}\left(s_{i(j+1)}\right)$ as initial point of $\tilde{\eta}_{i(j+1)}, \tilde{\gamma}_{i(j+1)}$; so on.

Consider now the straight-line homotopy between $\tilde{\gamma}, \tilde{\eta}$ keeping fixed the points $\tilde{s}_{i j} \in \tilde{\gamma}_{i} \cap \tilde{\eta}_{i}$. By Monodromy Theorem (2.5.10), calling the homotopy $\tilde{H}: \tilde{\eta} \simeq \tilde{\gamma}(\operatorname{rel} . \tilde{Q})$, it follows that $\eta_{i j} \simeq$ $\gamma_{i j}\left(\right.$ rel. $\left.\left\{s_{i j}, s_{i(j+1)}\right\}\right)$ for all $p_{1} \leq i \leq p_{1}+p_{2}-1,0 \leq j \leq k p_{2}+1$. Then, by homotopy invariance of path multiplication (2.5.8) between the homotopies $H_{i j}: \eta_{i j} \simeq \gamma_{i j}\left(\operatorname{rel} .\left\{s_{i j}, s_{i(j+1)}\right\}\right)$, we get the homotopy $H: \eta \simeq \gamma($ rel. $Q)$. Since the lifts $\tilde{\eta}, \tilde{\gamma}$ are uniques for any point in the fiber $\pi^{-1}\left(s_{i j}\right)$, these simple curves commute with Deck transformations. It follows that the homotopy $\tilde{H}$ commute with respect to the group of Deck transformations. Therefore the homotopy $H$ is well define, as we want.

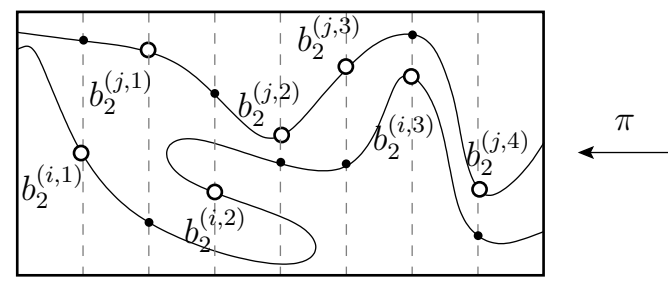

$\mathbb{T}^{2}$

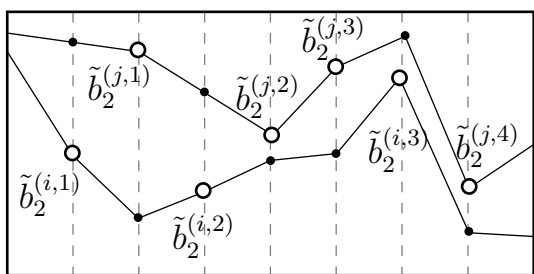

$\mathbb{R}^{2}$

Figure 4.15: Lift of two piecewise geodesic curve from simple arcs $\gamma_{i}$ in the $\boldsymbol{b}$ - strip.

Case 2. Assume that the simple arc $\gamma_{p_{1}+p_{2}-1}$ contains all $b_{1}$ 's on $\boldsymbol{b}$-strip (i.e., $\ell_{b}^{p_{1}+p_{2}-1}=\ell_{b} \geq 1$ ). From 4.2.10(ii), each $\gamma_{i}=\Gamma_{b} \cap \boldsymbol{b}$ has $k$ points in the region $\boldsymbol{b}_{\mathbf{2}}$. Let $\left\{b_{2}^{(i, 1)}, b_{2}^{(i, 2)}, \ldots, b_{2}^{(i, k)}\right\} \subset \gamma_{i} \cap \Sigma_{b_{2}}$ be such a set, $p_{1} \leq i \leq p_{1}+p_{2}-1$. The points $\gamma_{i} \cap \Sigma_{b_{2}}$ satisfy that $\pi_{y}\left(\tilde{b}_{2}^{(i, 1)}\right)<\cdots<\pi_{y}\left(\tilde{b}_{2}^{(i, k)}\right)$ in $D$. Furthermore, $\bigcup_{i=p_{1}}^{p_{1}+p_{2}-2}\left(\gamma_{i} \cap \Sigma_{b_{2}}\right) \subset\left[f\left(\boldsymbol{a}_{1}\right)\right]^{\circ}$. Denote by $\delta=p_{1}+p_{2}-1$. Since $\ell_{b} \geq 1$, $\left\{b_{1}^{(\delta, 1)}, b_{1}^{(\delta, 2)}, \ldots, b_{1}^{\left(\delta, \ell_{b}\right)}\right\} \subset \gamma_{\delta}$ where

$$
\pi_{y}\left(\tilde{b}_{1}^{(\delta, 1)}\right)<\cdots<\pi_{y}\left(\tilde{b}_{1}^{\left(\delta, \ell_{b}\right)}\right)<\pi_{y}\left(\tilde{b}_{2}^{(\delta, 1)}\right)<\cdots<\pi_{y}\left(\tilde{b}_{2}^{(\delta, k)}\right),
$$

In particular, if

$$
\begin{aligned}
\ell_{b} \leq k ; \quad\left\{b_{1}^{(\delta, 1)}, \ldots, b_{1}^{\left(\delta, \ell_{b}\right)}, b_{2}^{(\delta, 1)}, \ldots, b_{1}^{\left(\delta, k-\ell_{b}\right)}\right\} & \subset\left[f\left(\boldsymbol{a}_{\mathbf{1}}\right)\right]^{\circ}, \\
\left\{b_{2}^{\left(\delta, k-\ell_{b}+1\right)}, \ldots, b_{2}^{(\delta, k)}\right\} & \subset\left[f\left(\boldsymbol{b}_{\mathbf{1}}\right)\right]^{\circ}, \\
\ell_{b}>k ; \quad\left\{b_{1}^{(\delta, 1)}, \ldots, b_{1}^{(\delta, k)}\right\} & \subset\left[f\left(\boldsymbol{a}_{\mathbf{1}}\right)\right]^{\circ}, \\
\left\{b_{1}^{(\delta, k+1)}, \ldots, b_{1}^{\left(\delta, k+\ell_{b}\right)}, b_{2}^{(\delta, 1)}, \ldots, b_{2}^{(\delta, k)}\right\} & \subset\left[f\left(\boldsymbol{b}_{\mathbf{1}}\right)\right]^{\circ} .
\end{aligned}
$$

In both cases, $\gamma_{\delta}$ moves through the $\boldsymbol{b}$-strip going from band $f\left(\boldsymbol{a}_{\mathbf{1}}\right)$ to $f\left(\boldsymbol{b}_{\mathbf{1}}\right)$. Furthermore, $\gamma_{\delta} \succ \gamma_{p_{1}+p_{2}-2} \succ \cdots \succ \gamma_{p_{1}}$ in $\boldsymbol{b}$. Once this is clear, we see that the conditions are similar to the previous case, so we proceed as before. 


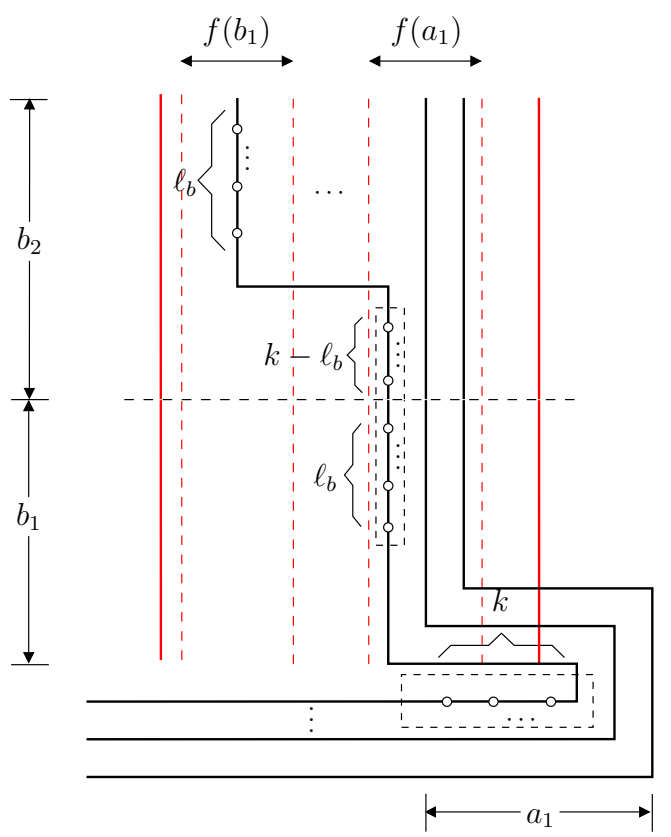

Figure 4.16: Illustration of the case $0<\ell_{b} \leq k$, for which all $b_{1}$ 's points belong to b-arc $\gamma_{p_{1}+p_{2}-1}$.

Before we proceed with the case 3, we will prove the following two propositions.

Notation. We denote by $\Gamma=\gamma_{i_{0}} \cup \cdots \gamma_{i_{n}}$ to the set the $b$-arcs with $b_{1}$ 's, $p_{1} \leq i_{0}<\cdots<i_{n} \leq$ $p_{1}+p_{2}-1$.

Proposition 4.4.4. Let $\Gamma=\gamma_{i_{0}} \cup \cdots \gamma_{i_{n}}, p_{1} \leq i_{0}<\cdots<i_{n} \leq p_{1}+p_{2}-1$. Then, $L_{i_{n}} \leq \cdots \leq L_{i_{0}}$. Proof. The result follows directly from $T_{i_{n}} \subset T_{i_{n-1}} \subset \cdots \subset T_{i_{n}}$ in 4.3.1(G3).

Proposition 4.4.5. Let $\Gamma=\gamma_{i_{0}} \cup \cdots \gamma_{i_{n}}, p_{1} \leq i_{0}<\cdots<i_{n} \leq p_{1}+p_{2}-1$. Let

$$
K_{i_{0}}=\left\{\begin{array}{ll}
L_{i_{0}} & ; L_{i_{1}}<L_{i_{0}}, \\
M_{i_{0}} & ; L_{i_{1}}=L_{i_{0}} .
\end{array} \quad \text {, and } \quad K_{i_{n+1}}= \begin{cases}M_{i_{n+1}} & ; M_{i_{n+1}}<K_{i_{n}} \\
K_{i_{n}} & ; M_{i_{n+1}}>K_{i_{n}} .\end{cases}\right.
$$

Then,

(i) $L_{i_{m}}<K_{i_{m}} \leq M_{i_{m}}, m=1, \ldots, n$;

(ii) $K_{i_{n}} \leq \cdots \leq K_{i_{0}}$.

Proof. We will use induction on $n$. Suppose first for $n=1$, thus

$$
K_{i_{0}}=\left\{\begin{array}{ll}
L_{i_{0}} & ; L_{i_{1}}<L_{i_{0}}, \\
M_{i_{0}} & ; L_{i_{1}}=L_{i_{0}} .
\end{array} \text { and } \quad K_{i_{1}}= \begin{cases}M_{i_{1}} & ; M_{i_{1}}<K_{i_{0}} \\
K_{i_{0}} & ; M_{i_{1}}>K_{i_{0}}\end{cases}\right.
$$

By Proposition 4.4.4, we have two cases:

If $L_{i_{1}}<L_{i_{0}}$, then $K_{i_{0}}=L_{i_{0}}$. It follows:
(i) $K_{i_{1}}=\underbrace{M_{i_{1}}>L_{i_{1}}}_{4.3 .1(G 3)}$
(ii) $\quad K_{i_{1}}=M_{i_{1}}<K_{i_{0}}$
$K_{i_{1}}=K_{i_{0}}=L_{i_{0}}>L_{i_{1}}$
$K_{i_{1}}=K_{i_{0}}$ 
If $L_{i_{1}}=L_{i_{0}}$, then $K_{i_{0}}=M_{i_{0}}$. It follows:

$$
\begin{array}{rlrl}
\text { (i) } K_{i_{1}} & =\underbrace{M_{i_{1}}>L_{i_{1}}}_{4.3 .1(G 3)} & \text { (ii) } & K_{i_{1}}=M_{i_{1}}<K_{i_{0}} \\
K_{i_{1}} & =K_{i_{0}}=\underbrace{M_{i_{0}}>L_{i_{0}}}_{4.3 .1(G 3)}=L_{i_{1}} & K_{i_{1}}=K_{i_{0}}
\end{array}
$$

Assume now the result for $n(n>1)$, this is $L_{i_{n}}<K_{i_{n}} \leq M_{i_{n}}$ and $K_{i_{n}} \leq K_{i_{n-1}}$. To show for $n+1$, we consider

$$
K_{i_{n+1}}= \begin{cases}M_{i_{n+1}} & ; M_{i_{n+1}}<K_{i_{n}} \\ K_{i_{n}} & ; M_{i_{n+1}}>K_{i_{n}}\end{cases}
$$

Solving by cases,
(1) $\quad K_{i_{n+1}}=\underbrace{M_{i_{n+1}}>L_{i_{n+1}}}_{4.3 .1(G 3)}$,
(ii) $K_{i_{n+1}}=M_{i_{n+1}}<K_{i_{n}}$,
$K_{i_{n+1}}=K_{i_{n}} \underset{(*)}{>} \underbrace{L_{i_{n}} \geq L_{i_{n+1}}}_{4.3 .1(G 3)}$.
$K_{i_{n+1}}=K_{i_{n}}$

$(*)$ : By hypothesis of induction.

(2) $K_{i_{n+1}}=M_{i_{n+1}}$,

$K_{i_{n+1}}=K_{i_{n}}<M_{i_{n+1}}$.

From (1) and (2) it follows (i).

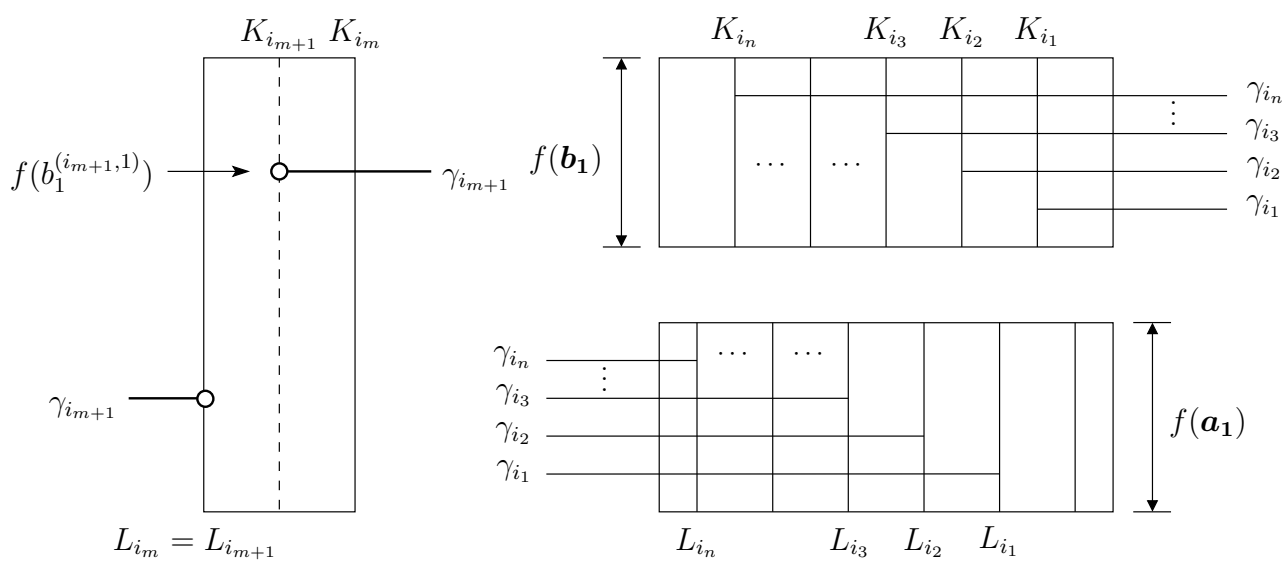

(a)

(b)

Figure 4.17: (a) The picture illustrate two continuous b-arcs $\gamma_{i}, \gamma_{i_{m+1}}$ with $b_{1}$ 's points in them such that $L_{i-m}=L_{i_{m+1}}<M_{i_{m+1}}<M_{i_{m}}$. (b) The bounds satisfy that $L_{i_{m}}<K_{i_{m}}, m=1, \ldots, n$ with $p_{1} \leq i_{0}<\cdots<$ $i_{n} \leq p_{1}+p_{2}-1$.

The following two propositions correspond to the versions on the $a$-strip and their proofs follow in a similar way.

Proposition 4.4.6. Let $\Gamma=\gamma_{i_{0}} \cup \cdots \gamma_{i_{n}}, 0 \leq i_{0}<\cdots<i_{n} \leq p_{1}-1$. Then, $E_{i_{n}} \leq \cdots \leq E_{i_{0}}$. 
Proposition 4.4.7. Let $\Gamma=\gamma_{i_{0}} \cup \cdots \gamma_{i_{n}}, 0 \leq i_{0}<\cdots<i_{n} \leq p_{1}-1$. Let

$$
G_{i_{0}}=\left\{\begin{array}{ll}
E_{i_{0}} & ; E_{i_{1}}<E_{i_{0}}, \\
F_{i_{0}} & ; E_{i_{1}}=E_{i_{0}} .
\end{array} \quad \text { and } \quad G_{i_{n+1}}= \begin{cases}F_{i_{n+1}} & ; F_{i_{n+1}}<G_{i_{n}} \\
G_{i_{n}} & ; F_{i_{n+1}}>G_{i_{n}}\end{cases}\right.
$$

Then,

(i) $E_{i_{m}}<G_{i_{m}} \leq F_{i_{m}}, m=1, \ldots, n$;

(ii) $G_{i_{n}} \leq \cdots \leq G_{i_{0}}$.

Case 3. Suppose that $p_{2}>1$ and $\ell_{b} \geq 1$ (not all $b_{1}$ 's are in $\gamma_{p_{1}+p_{2}-1}$ ). Let $R=\boldsymbol{b}$. Assume that $\gamma_{i},\left(p_{1} \leq i \leq p_{1}+p_{2}-2\right)$ is the first $b$-arc with $b_{1}$ 's points. If $p_{1}<i$, the arcs with index from $p_{1}$ to $i-1$ only have $b_{2}$ 's points contained in the interior of the closed rectangular region $f\left(\boldsymbol{a}_{\mathbf{1}}\right)$.

PART I. Suppose $\Gamma=\gamma_{i_{0}} \cup \cdots \gamma_{i_{n}}$ with $p_{1} \leq i=i_{0}<\cdots<i_{n} \leq p_{1}+p_{2}-1$. Since $L_{i_{n}} \leq \cdots \leq L_{i_{0}}$ by 4.4.5, we may assume without lost of generality that the $\operatorname{arcs} \Gamma_{i_{m}}=\gamma_{i_{m}} \cup \gamma_{i_{m}+1} \cup \cdots \cup \gamma_{i_{(m+1)}-1}$ intersect $L_{i_{m}}(m=0, \ldots, n-1)$ and $\Gamma_{i_{n}}=\gamma_{i_{n}} \cup \cdots \cup \gamma_{p_{1}+p_{2}-1}$ intersect $L_{i_{n}}$.

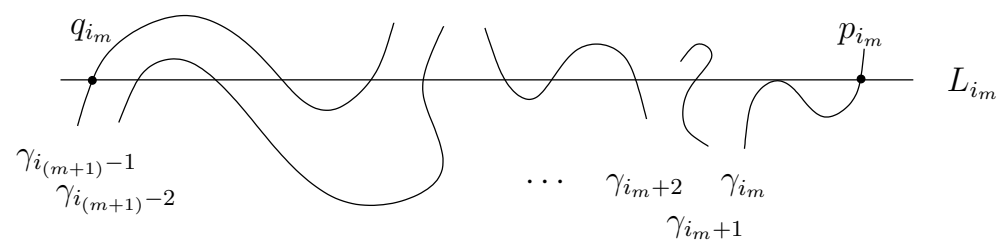

Figure 4.18: Representation of the points $p_{i_{m}}, q_{i_{m}} ;$ for $(m=0, \ldots, n)$.

Let $L_{i_{m}}^{*} \subset L_{i_{m}}$ be the closed arc for which the points $\Gamma_{i_{m}} \cap L_{i_{m}} \subset L_{i_{m}}^{*}$ (see figure 4.18). Call $\left\{p_{i_{m}}, q_{i_{m}}\right\}=\partial L_{i_{m}}^{*}$ for which $p_{i_{m}} \in L_{i_{m}} \cap \gamma_{i_{m}}$ and $q_{i_{m}} \in L_{i_{m}} \cap \gamma_{i_{m+1}-1}$. Now, let consider us $\psi_{i_{m}}$ be a simple closed geodesic arc with $\partial \psi_{i_{m}}=\left\{q_{i_{m}}, p_{i_{m+1}}\right\}$ such that $\psi_{i_{m}} \succ \gamma_{i_{(m+1)}-1}$ in the closed disk bounded by the Jordan curve contained in $\partial^{i n}(R) \cup L_{i_{m}} \cup L_{i_{m+1}}$ (see figure 4.19). In particular, if $L_{i_{m}}=L_{i_{m+1}}=L$ then $\psi_{i_{m}} \subset L$. This follows from $\pi_{x}\left(\tilde{q}_{i_{m+1}}\right)<\pi_{x}\left(\tilde{q}_{i_{m}}\right)$.

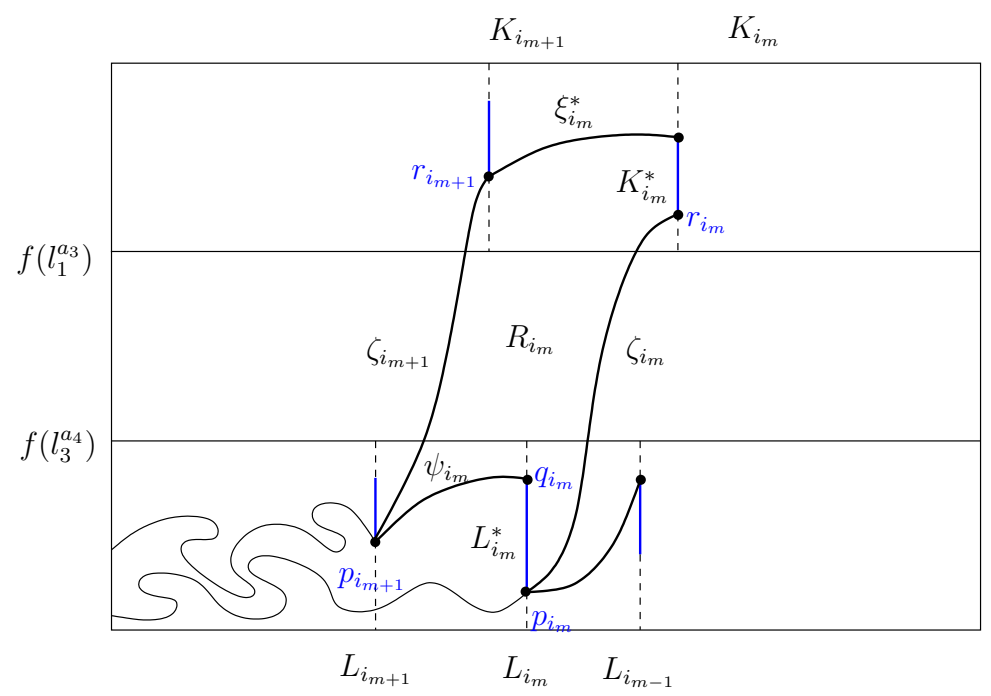

Figure 4.19: Representation of the simple closed geodesic arcs $\psi_{i_{m}}, \zeta_{i_{m}} ;$ for $(m=0, \ldots, n)$.

On the other hand, consider the leaves $K_{i_{0}}, \ldots, K_{i_{n}}$ in the Propositions 4.4.4, 4.4.5 for which $K_{i_{n}} \leq \cdots \leq K_{i_{0}}$ and $L_{i_{m}}<K_{i_{m}} \leq M_{i_{m}}$ for $m=1, \ldots, n$. For $m=0$, notice that from 4.3.1(G3) and (4.7), we have that (see figure \#): 


$$
\begin{aligned}
& \text { if } L_{i_{1}}<L_{i_{0}} \text {, then } L_{i_{1}}<K_{i_{1}} \leq K_{i_{0}}=L_{i_{0}}<M_{i_{0}} \text { and } \\
& \text { if } L_{i_{1}}=L_{i_{0}} \text {, then } L_{i_{0}}=L_{i_{1}}<K_{i_{1}} \leq K_{i_{0}}=M_{i_{0}} \text {. }
\end{aligned}
$$

Being $L_{i_{1}}<L_{i_{0}}$, we assign a new bound $K_{i_{0}}^{\prime}$, as follows

$$
K_{i_{0}}^{*}:=\text { The leaf more close to } K_{i_{0}} \text { by its rights containing an orbit point }\left(b_{1} \text { or } b_{2}\right) \text {. }
$$

Consider the pieces of the $\operatorname{arcs} \gamma_{i_{m}}(m=0, \ldots, n)$ between the points $\left\{b_{1}^{\left(i_{m}, 1\right)}, \gamma_{i_{m}} \cap l_{3}^{R}\right\}$. We will not change the name of the curve. Let $\xi_{i_{n}}$ be a simple geodesic arc with endpoints $\partial \xi_{i_{n}}=$ $\left\{r_{i_{n}}, b_{1}^{\left(i_{n}, 1\right)}\right\}$ such that $r_{i_{n}} \in\left[K_{i_{n}} \cap f\left(\boldsymbol{b}_{\mathbf{1}}\right)\right]^{\circ}$. It follows that $\gamma_{i_{n}} \cup \xi_{i_{n}}$ is a simple arc with endpoints in $K_{i_{n}}, l_{3}^{R}$ (see figure 4.20). We assume that $\xi_{i_{n}} \cap\left(\gamma_{i_{0}} \cup \cdots \cup \gamma_{i_{n-1}}\right)=\emptyset$.

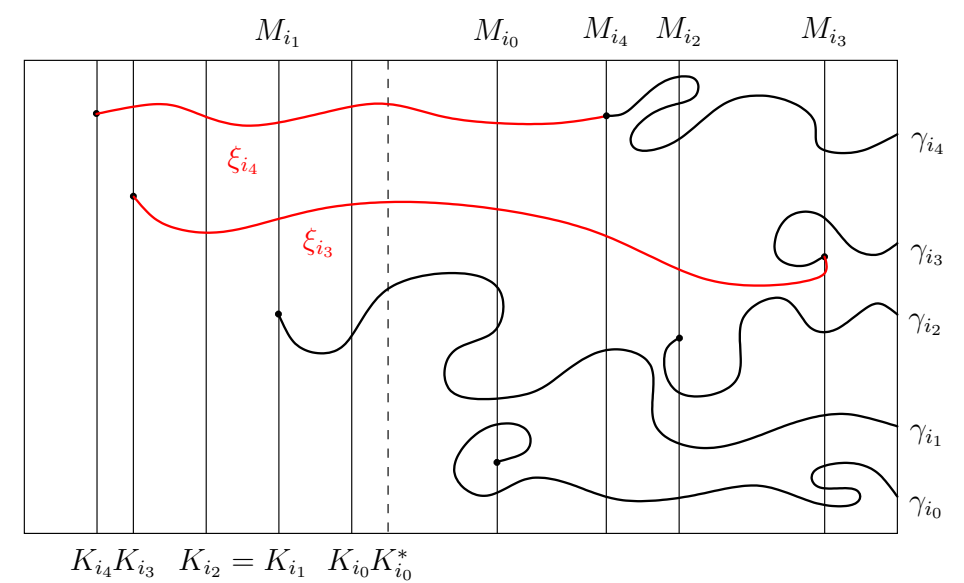

Figure 4.20: Extension on $\boldsymbol{b}$ of the arcs $\gamma_{i_{m}}(m=0, \ldots, n)$ with $p_{1} \leq i=i_{0}<\cdots<i_{n} \leq p_{1}+p_{2}-1$.

Let $K_{i_{n}}^{*} \subset K_{i_{n}}$ such that $\partial K_{i_{n}}^{*}=\left\{r_{i_{n}}, l_{4}^{R} \cap K_{i_{n}}\right\}$. From the fact that $\left[K_{i_{n}}^{*} \cup \xi_{i_{n}} \cup{\gamma_{i_{n}}}\right]^{\circ}$ is a cross-cut in $R$ with endpoints $l_{4}^{R} \cap K_{i_{n}}, l_{3}^{R} \cap \gamma_{i_{n}}$, we have by $\theta$-curve Theorem 2.5.4, $\left[R \backslash \mathbb{D}_{i_{n+1}}\right]^{\circ} \supset$ $\left[\gamma_{i_{0}} \cup \cdots \cup \gamma_{i_{n-1}}\right]^{\circ}$. Let $\xi_{i_{n-1}} \subset\left[R \backslash \mathbb{D}_{i_{n+1}}\right]^{\circ}$ extending $\gamma_{i_{n-1}}$ as before (i.e., $\partial \xi_{i_{n-1}}=\left\{r_{i_{n-1}}, b_{1}^{\left(i_{n-1}, 1\right)}\right\}$ ). It follows that $\left[R \backslash\left(\mathbb{D}_{n} \cup \mathbb{D}_{n-1}\right)\right]^{\circ} \supset\left[\gamma_{i_{0}} \cup \cdots \cup \gamma_{i_{n-2}}\right]^{\circ}$. Thus,

$$
K_{i_{n-1}}^{*} \cup\left(\xi_{i_{n}} \cup \gamma_{i_{n}}\right) \cup\left(\xi_{i_{n-1}} \cup \gamma_{i_{n-1}}\right)
$$

for $\partial K_{i_{n-1}}^{*}=\left\{K_{i_{n-1}} \cap \xi_{i_{n-1}}, K_{i_{n-1}} \cap \xi_{i_{n}}\right\}$ is a cross-cut in $\left[R \backslash \mathbb{D}_{i_{n+1}}\right]^{\circ}$. If $K_{i_{n}}=K_{i_{n-1}}$, we choose $r_{i_{n}}, r_{i_{n-1}}$ so that $\pi_{x}\left(\tilde{r}_{i_{n}}\right)<\pi_{x}\left(\tilde{r}_{i_{n-1}}\right)$. We continue in the same way until $R \backslash \bigcup_{m=1}^{n+1} \mathbb{D}_{i_{m}}$. For $m=0$, we extend the $\gamma_{i_{0}}$ arc according to (4.9). When When $K_{i_{0}}=L_{i_{0}}$, we do it until $K_{i_{0}}$, otherwise $K_{i_{0}}=M_{i_{0}}$. We note that $\bigcap_{m=1}^{n+1} \mathbb{D}_{i_{m}}^{\circ} \cap \Sigma_{b}=\emptyset$.

PART II. From this it is not hard to see that there exist the piecewise closed curve $\xi, \psi$ defined by

$$
\begin{aligned}
& \psi \subset\left(\bigcap_{m=1}^{n} \psi_{i_{m}} \cup L_{i_{m}}^{*}\right) \cup\left(L_{i_{0}} \cup \gamma_{i_{0}}\right) \cup \gamma_{p_{1}+p_{2}-1} \\
& \xi \subset\left(\bigcap_{m=0}^{n} \xi_{i_{m}} \cup K_{i_{m}}^{*}\right) \cup \gamma_{i_{0}}
\end{aligned}
$$

such that $\xi^{\circ}, \psi^{\circ} \subset R^{\circ}$ and them separate $R$. It follows by 2.5.5 that $R_{0}^{\circ}, R_{1}^{\circ}, R_{2}^{\circ}$ are bounded components of $R \backslash(\partial R \cup \xi \cup \psi)$. Notice that 


$$
R_{0} \cap \mathcal{O}=f\left(\boldsymbol{a}_{\mathbf{1}}\right) \cap \mathcal{O} ; \quad R_{1}^{\circ} \cap \mathcal{O}=\emptyset ; \quad R_{2} \cap \mathcal{O}=f\left(\boldsymbol{b}_{\mathbf{1}}\right) \cap \mathcal{O} .
$$

Let $\zeta_{i_{m}}$ be a simple closed geodesic arc with $\partial \zeta_{i_{m}}=\left\{p_{i_{m}}, r_{i_{m}}\right\}$ such that $\zeta_{i_{m}}^{\circ} \subset R_{1}^{\circ}$ and $\zeta_{i_{m}} \succ$ $\psi_{i_{m}}$. Since $p_{i_{m}} \in \xi \cap L_{i_{m}}$ and $r_{i_{m}} \in \psi \cap K_{i_{m}}$, for $m=0, \ldots, n$ we can choose them disjoint in $R_{1}^{\circ}$, as follows: We start with $\zeta_{i_{0}}$ with endpoints in $L_{i_{0}}, K_{i_{0}}$ (or $K_{i_{0}}^{\prime}$ ). By (4.9), $L_{i_{0}}<K_{i_{0}} \leq K_{i_{0}}^{\prime}$. Applying 2.5.3 to $R_{1}$, we obtain domains $R_{i_{0}}^{(1)},\left[R_{1} \backslash R_{i_{0}}^{(1)}\right]^{\circ}$ bounded by $R_{1} \backslash\left(\partial R_{1} \cup \zeta_{i_{0}}\right)$. From Propositions 4.4.4, 4.4.5; $L_{i_{1}} \leq L_{i_{0}}$ and $K_{i_{1}} \leq K_{i_{0}}$. Then we can choose $\zeta_{i_{2}}$ with $\partial \zeta_{i_{2}}=\left\{p_{i_{2}}, r_{i_{2}}\right\}$ so that $\zeta_{i_{2}}^{\circ} \subset\left[R_{1} \backslash R_{i_{0}}^{(1)}\right]^{\circ}$. If $L_{i_{1}}<L_{i_{0}}$ and $K_{i_{1}}<K_{i_{0}}$ then its clear that $\zeta_{i_{0}}$ and $\zeta_{i_{2}}$ are disjoint. Otherwise, we have that $\pi_{x}\left(\tilde{r}_{i_{0}}\right)<\pi_{x}\left(\tilde{r}_{i_{1}}\right)$ and $\pi_{x}\left(\tilde{p}_{i_{0}}\right)<\pi_{x}\left(\tilde{p}_{i_{1}}\right)$. By 2.5.3, there exists $R_{i_{0}}^{\circ},\left[R_{1} \backslash\left(R_{i_{0}}^{(1)} \cup R_{i_{1}}^{(1)}\right)\right]^{\circ}$ bounded components of $R_{1} \backslash\left(\partial R_{1} \cup\left(\zeta_{i_{0}} \cup \zeta_{i_{1}}\right)\right)$; and so on.

Thus, $\left[R_{i_{0}}^{(1)}\right]^{\circ}, \ldots,\left[R_{i_{n+1}}^{(1)}\right]^{\circ}$ are bounded components of $\left[R_{1} \backslash\left(\partial R_{1} \cup\left(\bigcup_{m=0}^{n} \zeta_{i_{m}}\right)\right)\right]^{\circ}$.

PART III. Done this, we will define the $\left(p_{1}, p_{2}\right)$-curve $\eta$ candidate to be unimodal. In particular, we will define the piecewise geodesic arcs $\eta_{i} \subset \eta$ along of $\gamma_{i}$ for which $\gamma_{i} \cap \Sigma_{b} \subset \eta_{i} \cap \gamma_{i}, i=$ $p_{1}, \ldots, p_{1}+p_{2}-1$. In what follows, we will define the set of vertices $P$ of $\eta$. For this, we begin by redefining $\gamma_{i}$ as follows:

$$
\gamma_{i_{m}}:=\gamma_{i_{m}}^{(1)} \cap \zeta_{i_{m}} \cup \xi_{i_{m}} \cup \gamma_{i_{m}}^{(2)} ; m=0, \ldots, n,
$$

where $\partial \gamma_{i_{m}}^{(1)}=\left\{l_{1}^{R}, p_{i_{m}}\right\}$ and $\partial \gamma_{i_{m}}^{(2)}=\left\{b_{1}^{\left(i_{m}, 1\right)}, l_{3}^{R}\right\}$. Then, by 2.5.5 we have $n+1$ bounded components in $R$ that we denote by $R_{i_{0}}, \ldots, R_{i_{n+1}}$. We note that

$$
\begin{array}{ll}
\mathbb{R}_{i_{0}} & \supset \gamma_{p_{1}} \cup \cdots \cup \gamma_{i_{0}-1} ; \\
\mathbb{R}_{i_{m+1}} & \supset \gamma_{i_{m}} \cup \cdots \cup \gamma_{i_{(m+1)}-1}, \quad m=0, \ldots, n-1 ; \\
\mathbb{R}_{i_{n+1}} & \supset \gamma_{i_{n}} \cup \cdots \cup \gamma_{p_{1}+p_{2}-1} .
\end{array}
$$

Let

$$
\Lambda=\left(\bigcap_{m=0}^{n} K_{i_{m}}\right) \cup\left\{\text { leaves that contains } b_{1} \text { 's or } b_{2} \text { 's }\right\}
$$

and let us consider for $m=0, \ldots, n$ the subsets

$$
\Lambda_{i_{m}}=\left\{\lambda \in \Lambda \mid \lambda \leq L_{i_{m}} \wedge \lambda \geq K_{i_{m}}\right\} .
$$

Thus, let us define the sets:

$$
\begin{aligned}
& P_{i_{0}}=\left\{s_{p_{1}}, \ldots, s_{i_{0}-1}\right\} ; \\
& P_{i_{m+1}}=\left\{s_{i_{m}}, \ldots, s_{i_{(m+1)}}-1\right\}, \quad m=0, \ldots, n-1 ; \\
& P_{i_{n+1}}=\left\{s_{i_{n}}, \ldots, s_{p_{1}+p_{2}-1}\right\} \text {; }
\end{aligned}
$$

so that $\Sigma_{b} \subset P=\bigcup_{m=0}^{n+1} P_{i_{m}}$.

Since $\gamma_{p_{1}} \prec \cdots \prec \gamma_{p_{1}+p_{2}-1}$, fixed $\Sigma_{b} \subset P$ we may choose the rest of points in $P$, so that for all $\lambda \in \Lambda$ the chosen points $\lambda \cap P$ are ordered. We can do this separately in $f\left(\boldsymbol{a}_{\mathbf{1}}\right)$ and $f\left(\boldsymbol{b}_{\mathbf{1}}\right)$. More 
precisely, for $\left.\lambda \in \Lambda\right|_{f\left(\boldsymbol{a}_{1}\right)}$ the sets:

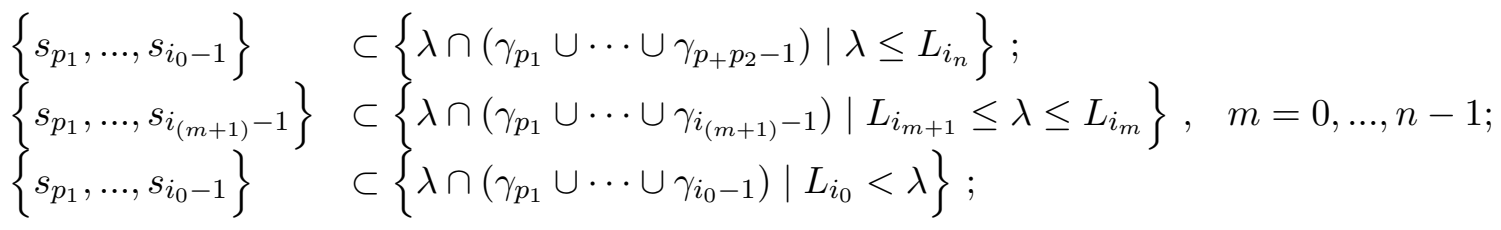

satisfy that the points in any of them are horizontal ordered in a fundamental domain in $\mathbb{R}^{2}$. Thus, in the first set have that $\pi_{x}\left(\tilde{s}_{p_{1}}\right)<\pi_{x}\left(\tilde{s}_{p_{1}+1}\right)<\cdots<\pi_{y}\left(\tilde{s}_{p_{1}+p_{2}-1}\right)$. Similarly, for $\left.\lambda \in \Lambda\right|_{f\left(\boldsymbol{b}_{\mathbf{1}}\right)}$ the sets:

$$
\begin{cases}\left.t_{i_{m+1}}, \ldots, t_{p_{1}+p_{2}-1}\right\} & \subset\left\{\lambda \cap\left(\gamma_{i_{m+1}} \cup \cdots \cup \gamma_{p_{1}+p_{2}-1}\right) \mid K_{i_{m+1}} \leq \lambda \leq K_{i_{m}}\right\}, \quad m=0, \ldots, n-1 ; \\ \left.t_{i_{0}}, \ldots, s_{p_{1}+p_{2}-1}\right\} & \subset\left\{\lambda \cap\left(\gamma_{i_{0}} \cup \cdots \cup \gamma_{p_{1}+p_{2}-1}\right) \mid K_{i_{0}} \geq \lambda\right\} .\end{cases}
$$

Once we found $P$ set, we can define the simple closed curve $\eta$. For $i_{0}, \ldots, i_{n}$ with $p_{1} \leq i_{0}<\cdots<$ $i_{n} \leq p_{1}+p_{2}-1$. Let

$$
\eta_{i_{m}}=\eta_{i_{m}}^{(1)} \cup \zeta_{i_{m}} \cup \eta_{i_{m}}^{(2)}
$$

with endpoints $\partial \eta_{i_{m}}^{(1)}=\left\{l_{1}^{R}, p_{i_{m}}\right\}, \partial \eta_{i_{m}}^{(2)}=\left\{r_{i_{m}}, l_{3}^{R}\right\}$ and vertices $\Lambda_{i_{m}} \cap \gamma_{i_{m}} \subset P_{i_{m}}$. We note that $\eta_{i_{m}}$ is a piecewise geodesic arc along $\gamma_{i_{m}}$ such that $\sigma_{b} \cap \gamma_{i_{m}} \subset \eta_{i_{m}}$. We can proceed in similar way to define the $\eta_{i}$ arcs between $i_{0}, \ldots, i_{n}$.

Once we assume that outside of $\boldsymbol{b}$ the curve $\eta$ and $\gamma$ coincide, the relative homotopy follows as the Case 1.

Convention. For the following theorem, we assume an extension of Definition 4.2.4 for a closed disk or a closed topological disk. The result follows from $\theta$-curve Theorem 2.5.4.

Theorem 4.4.8 (Main Theorem). Let $(\mathcal{O}, \gamma)$ be a unfoldable orbit-curve pair. Then, $\mathcal{O}$ is a finite order periodic orbit.

Proof. Let $p_{1}>p_{2}$. Suppose that the pair $(\mathcal{O}, \gamma)$ is unfoldable. By Theorem 4.4.3 the pair $(\mathcal{O}, \gamma)$ is unimodal. Then, we can assume that $\gamma$ is a oriented simple closed curve containing $\mathcal{O}$ which have $p_{1} \operatorname{arcs} \boldsymbol{a}$-monotones, $p_{2}$ arcs $\boldsymbol{b}$-monotones, and the arcs that connect $\boldsymbol{a}$ to $\boldsymbol{b}$ are unimodal on $\boldsymbol{a}_{\mathbf{1}}$. Since the points of each periodic orbit are located in the regions $\boldsymbol{a}_{\mathbf{1}}, \boldsymbol{a}_{\mathbf{3}}, \boldsymbol{a}_{\mathbf{4}}, \boldsymbol{b}_{\mathbf{1}}$, and $\boldsymbol{b}_{\mathbf{2}}$, we can mark the parts in $\gamma$ where the points are placed, in the remaining parts the curve is left dotted (see figure $4.21(\mathrm{a})$ ).

From the Definition 3.1.1, we have that $f$ expands the strips $\boldsymbol{a}, \boldsymbol{b}$ preserving the monotonicity of the simple arcs in them. The images $f\left(\boldsymbol{a}_{\mathbf{3}}\right), f\left(\boldsymbol{a}_{\mathbf{4}}\right), f\left(\boldsymbol{b}_{\mathbf{2}}\right)$ cover the $\boldsymbol{a}$-strip and $f\left(\boldsymbol{a}_{\mathbf{1}}\right), f\left(\boldsymbol{b}_{\mathbf{1}}\right)$ cover the $\boldsymbol{b}$-strip. By 4.2.5 and 4.2.6, the monotonous pieces $\boldsymbol{a}_{\boldsymbol{i}} \cap \Gamma_{a}(i=1,3,4), \boldsymbol{b}_{\boldsymbol{j}} \cap \Gamma_{b}(j=1,2)$ and $\boldsymbol{a}_{\mathbf{1}} \cap\left(\Gamma_{a} \cup \Gamma_{b}\right)$ are expanded by $f$ preserving the monotonicity, so that the images of $f\left(\boldsymbol{a}_{\boldsymbol{i}} \cap \Gamma_{a}\right)$, $f\left(\boldsymbol{b}_{\boldsymbol{j}} \cap \Gamma_{b}\right)$ go from left to right in the $\boldsymbol{a}$-strip and from bottom to top in the $\boldsymbol{b}$-strip, respectively. Furthemore, $\left.f\left(\boldsymbol{a}_{\mathbf{1}} \cap\left(\Gamma_{a} \cup \Gamma_{b}\right)\right)=f\left(\boldsymbol{a}_{\mathbf{1}} \cap \Gamma_{a}\right) \cup f\left(\boldsymbol{a}_{\mathbf{1}} \cap \Gamma_{b}\right)\right)$ has two behaviors: the arcs in $\boldsymbol{a}_{\mathbf{1}} \cap \Gamma_{a}$ have endpoints in $l_{4}^{a_{1}}$ and $l_{2}^{a_{1}}$ and the arcs in $\boldsymbol{a}_{\mathbf{1}} \cap \Gamma_{b}$ (where $\left.\left(\boldsymbol{a}_{\mathbf{1}} \cap \Gamma_{b}\right)^{\circ} \subset \boldsymbol{a}_{\mathbf{1}}{ }^{\circ}\right)$ go in and out by $l_{4}^{a_{1}}$ (see figure 4.22(a)). Then, the image of the first go through $\boldsymbol{b}$ from $l_{1}^{b}$ to $l_{3}^{b}$ and the image of the seconds go in and out by $l_{1}^{b}$. In particular, $\left[f\left(\boldsymbol{a}_{\mathbf{1}} \cap \Gamma_{b}\right)\right]^{\circ} \subset \boldsymbol{b}^{\circ}$.

We more accurately illustrate the case $p_{1}>p_{2}$ (see figure 4.21(b)). Note that we only mark the parts of the $f(\gamma)$ curve that intercept the regions that may contain orbit points. 

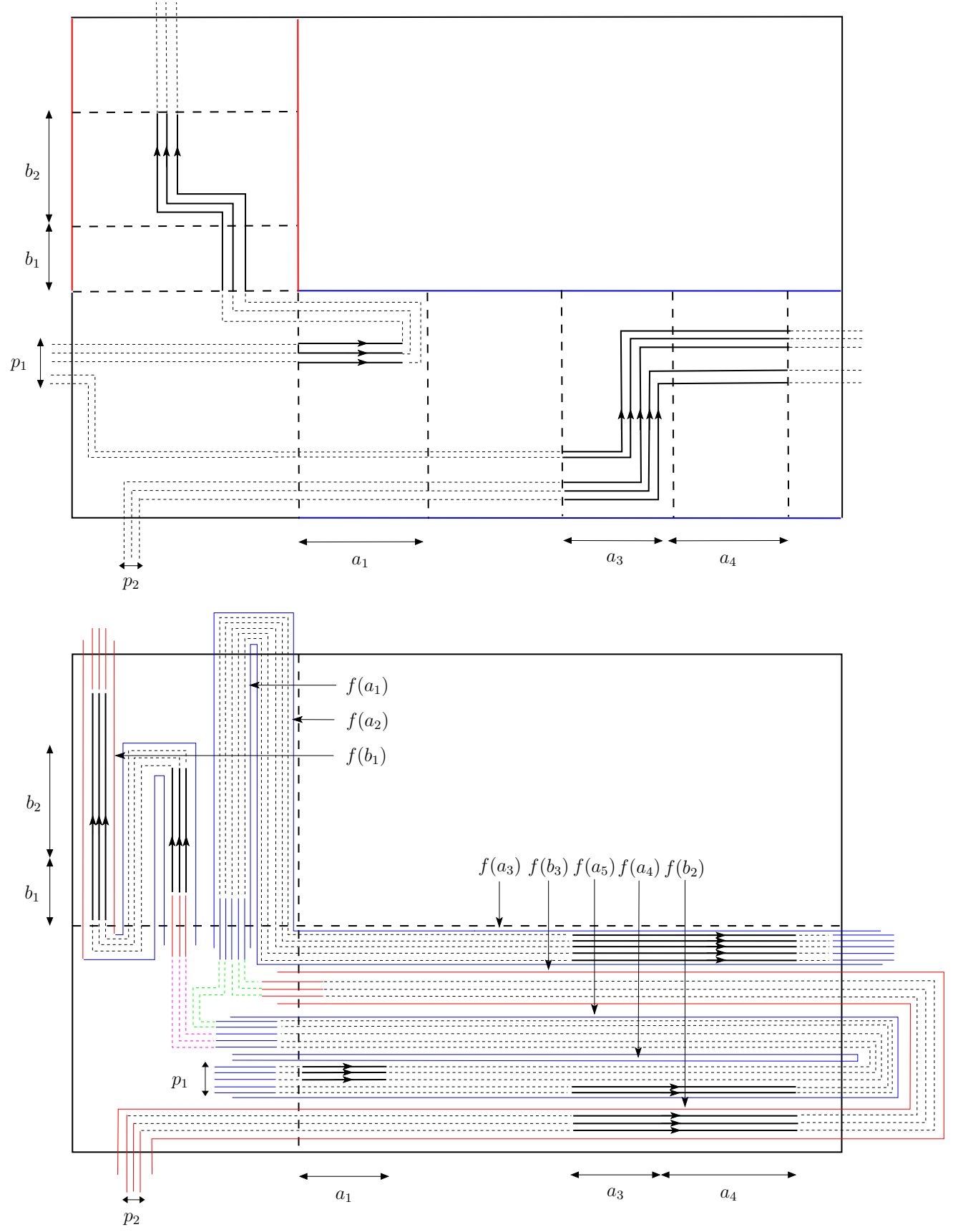

Figure 4.21: (a) (figure at the top) Representation of an unfondable pair $(\mathcal{O}, \gamma)$ for $p_{1}>p_{2}$. (b) (figure at the bottom) Image of the pair $(\mathcal{O}, \gamma)$ by $f$.

PART I. First we deform isotopically $f(\gamma)$ on the dotted parts. From Definition 3.1.1, the arcs $f\left(\Gamma_{a} \cap\left(\boldsymbol{a}_{\mathbf{1}} \cup \boldsymbol{a}_{\mathbf{2}}\right)\right)$ intersect transversally $l_{3}^{b}$. Then, for $\varepsilon>0$ large enough we define the closed disk $\mathbb{D}_{\varepsilon}$ for which $l_{3}^{b} \cap \mathbb{D}_{\varepsilon}$ is a topological diameter and $\left[\mathbb{V} \cap f\left(\Gamma_{a} \cap\left(\boldsymbol{a}_{\mathbf{1}} \cup \boldsymbol{a}_{\mathbf{2}}\right)\right]^{\circ} \subset \mathbb{D}_{\varepsilon}^{\circ}\right.$ (see figure \#). By the Corollary 2.5.2, there exist a isotopy of the identity with $\partial \mathbb{D}_{\varepsilon}$ pointwise fixed that carry each simple arc $c_{i} \subset \mathbb{D}_{\varepsilon} \cap f\left(\gamma_{i} \cap\left(\boldsymbol{a}_{\mathbf{1}} \cup \boldsymbol{a}_{\mathbf{2}}\right)\right)$ onto another $c_{i}^{\prime}$ in different sides of $l_{3}^{b} \cap \mathbb{D}_{\varepsilon}, i=p_{1}, \ldots, p_{1}+p_{2}-1$. Since $\left[f\left(\gamma_{i} \cap\left(\boldsymbol{a}_{\mathbf{1}} \cup \boldsymbol{a}_{\mathbf{2}}\right)\right) \cap \mathbb{D}_{\varepsilon}\right]^{\circ} \subset\left[\mathbb{D}_{\varepsilon}^{r i}\left(f\left(\gamma_{i+1} \cap\left(\boldsymbol{a}_{\mathbf{1}} \cup \boldsymbol{a}_{\mathbf{2}}\right)\right) \cap \mathbb{D}_{\varepsilon}\right)\right]^{\circ}$ for $i=0, \ldots, p_{1}-2$, we can begin the isotopy with $\gamma_{0}$ (see figure $4.22(\mathrm{a})$ ). 


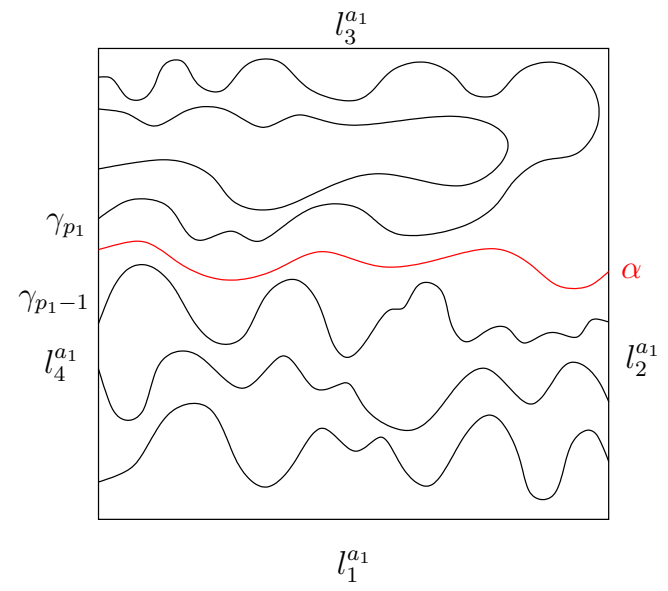

(a)



(b)

Figure 4.22: (a) a-monotone simple arcs and unimodal arcs in the region $\boldsymbol{a}_{\mathbf{1}}$. (b) Isotopic deformation of $f\left(\gamma_{i} \cap\left(\boldsymbol{a}_{\mathbf{1}} \cup \boldsymbol{a}_{\mathbf{2}}\right)\right)\left(i=0, \ldots, p_{1}-1\right)$.

Once this is done, the $\operatorname{arcs} f\left(\Gamma_{a} \cap\left(\boldsymbol{a}_{\mathbf{1}} \cup \boldsymbol{a}_{\mathbf{2}}\right)\right)$ (we call them the same way) are containing in $\boldsymbol{b}$. Since in $\boldsymbol{a}_{\mathbf{1}}$ by hypothesis $\gamma_{p_{1}}$ is unimodal and $\gamma_{p_{1}-1}$ is monotonous, by Theorem 2.5.6

$$
\left(a_{1}^{r i}\left(\gamma_{p_{1}}\right) \cap \boldsymbol{a}_{\mathbf{1}}\right) \cap\left(a_{1}^{l e}\left(\gamma_{p_{1}-1}\right) \cap \boldsymbol{a}_{\mathbf{1}}\right),
$$

is a closed topological disk. Then, we can choose a simple $\operatorname{arc} \alpha \subset \boldsymbol{a}_{\mathbf{1}}$ for which

$$
\alpha^{\circ} \subset\left[\left(a_{1}^{r i}\left(\gamma_{p_{1}}\right) \cap \boldsymbol{a}_{\mathbf{1}}\right) \cap\left(a_{1}^{l e}\left(\gamma_{p_{1}-1}\right) \cap \boldsymbol{a}_{\mathbf{1}}\right)\right]^{\circ},
$$

and $\partial \alpha \subset l_{4}^{a_{1}} \cup l_{2}^{a_{1}}$ (i.e., a cross-cut). We know that $f\left(\boldsymbol{a}_{\mathbf{1}}\right) \subset \boldsymbol{b}$ is a rectangular region by Corollary 4.2.7, then $f(\alpha)$ is a cross-cut in the $\boldsymbol{b}$-strip. Notice that $\mathbb{D}=b^{r i}(f(\alpha))$ is a closed topological disk as consequence of 2.5.4, for which

$$
f\left(\Gamma_{a} \cap\left(\boldsymbol{a}_{\mathbf{1}} \cup \boldsymbol{a}_{\mathbf{2}}\right)\right) \subset b^{r i}(f(\alpha)),
$$

with $\partial f\left(\gamma_{i} \cap\left(\boldsymbol{a}_{\mathbf{1}} \cup \boldsymbol{a}_{\mathbf{2}}\right)\right) \subset l_{1}^{b}, \gamma_{i} \subset \Gamma_{a}$. Let

$$
f\left(\gamma_{0} \cap\left(\boldsymbol{a}_{\mathbf{1}} \cup \boldsymbol{a}_{\mathbf{2}}\right)\right) \prec \cdots \prec f\left(\gamma_{p_{1}-1} \cap\left(\boldsymbol{a}_{\mathbf{1}} \cup \boldsymbol{a}_{\mathbf{2}}\right)\right),
$$

in $b^{r i}(f(\alpha))$ (see figure 4.23). As before there exist a isotopy of identity with $\partial b^{r i}(f(\alpha))$ pointwise fixed such that the arcs $f\left(\Gamma_{a} \cap\left(\boldsymbol{a}_{\mathbf{1}} \cup \boldsymbol{a}_{\mathbf{2}}\right)\right)$ are carried close enough to $l_{1}^{b}$. After this, again there exist a closed disk $\mathbb{D}_{\varepsilon}^{\prime}$ in which up to isotopy the simple arcs are "pushed" one by one to different sides of $l_{1}^{b}$. Now, the arcs are contained in the disk $\mathbb{V}$. Using the above procedure it is not hard to show that the $\Gamma_{a}$ arcs can be pushed up the points in $\boldsymbol{a}_{\mathbf{1}}$ and $\boldsymbol{a}_{\mathbf{3}}$. In this way, by Propositions 4.4.2(ii)(iv), the image arcs in the $\boldsymbol{a}$-strip are deform to:

$$
\begin{aligned}
f\left(\gamma_{i} \cap\left(\boldsymbol{b}_{\mathbf{2}} \cup \boldsymbol{b}_{\mathbf{3}}\right)\right) \cup f\left(\gamma_{\delta} \cap \boldsymbol{a}_{\mathbf{3}}\right), & i=p_{1}, \ldots, p_{1}+p_{2}-1, & \delta=\left(i+p_{2}\right) \bmod \left(p_{1}+p_{2}\right) ; \\
f\left(\gamma_{i} \cap\left(\boldsymbol{a}_{\mathbf{4}} \cup \boldsymbol{a}_{\mathbf{5}}\right)\right) \cup f\left(\gamma_{\delta} \cap \boldsymbol{a}_{\mathbf{3}}\right), & i=0, \ldots, p_{1}-p_{2}-1, & \delta=\left(i+p_{2}\right) \bmod \left(p_{1}+p_{2}\right) ;
\end{aligned}
$$

(i.e., that the $\gamma_{\delta}$ arcs take values between 0 and $p_{1}-1$ ). From now on, the path isotopy will be analyzed in the rectangular regions $\boldsymbol{a}_{\mathbf{3}} \cup \boldsymbol{a}_{\mathbf{4}}$ and $\boldsymbol{b}_{\mathbf{1}} \cup \boldsymbol{b}_{\mathbf{2}}$. We assume from (4.10)(4.11) the following 
notation

$$
f\left(\gamma_{i} \cup \gamma_{\delta}\right), \quad i \in\left\{p_{1}, \ldots, p_{1}+p_{2}-1\right\} \cup\left\{0, \ldots, p_{1}-p_{2}-1\right\} \text { with } \delta=\left(i+p_{2}\right) \bmod \left(p_{1}+p_{2}\right) .
$$

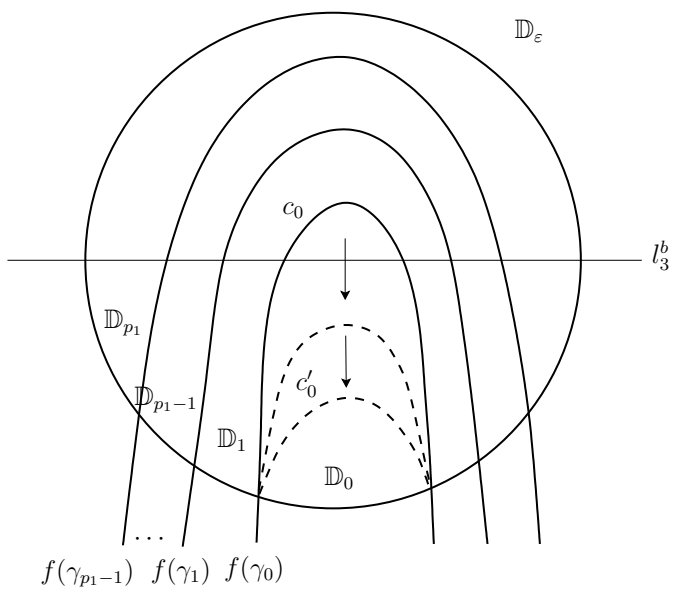

Figure 4.23: $\mathbb{D}_{\varepsilon}^{r i}\left(f\left(\gamma_{0}\right)\right) \subset \mathbb{D}_{\varepsilon}^{r i}\left(f\left(\gamma_{1}\right)\right) \subset \cdots \subset \mathbb{D}_{\varepsilon}^{r i}\left(f\left(\gamma_{p_{1}-1}\right)\right) \subset \mathbb{D}_{\varepsilon}$.

PART II. In the following part, we will complete the invariance of $\gamma$ up to relative isotopy. We consider three cases in relation to the definition of unfoldable pairs:

1) The periodic orbit has no points in $\boldsymbol{a}_{\mathbf{3}}$ (i.e., $\ell_{a}=0$ ).

2) The $a_{3}$ 's periodic orbit points are contained in the $a$-arc, $\gamma_{p_{1}-1}$ (i.e., $\ell_{a}=\ell_{a}^{p_{1}-1}>0$ ).

3) At least a $a_{3}$ point is contained in an $a$-arc $\gamma_{i}$ between $0, p_{1}-2$ (i.e., $\ell_{a}^{i}>0,0 \leq i \leq p_{1}-2$ ).

Note that the three cases are analogous for the arcs in the $b$-strip, the combination of any of the three conditions on the $a$-strip with any in $b$-strip will lead us to a finite order periodic orbit.

Case $\left(\ell_{a}=0\right)$. Let $\Sigma_{a_{3}}=\emptyset$. By Proposition 4.4.1(ii)

$$
f\left(\Sigma_{b_{2}}\right) \cup f\left(\Sigma_{a_{3}}\right) \cup f\left(\Sigma_{a_{4}} \cap\left(\cup_{i=0}^{p_{1}-p_{2}-1} \gamma_{i}\right)\right)=\Sigma_{a_{3}} \cup \Sigma_{a_{4}}
$$

it follows that

$$
f\left(\Sigma_{b_{2}}\right) \cup f\left(\Sigma_{a_{4}} \cap\left(\cup_{i=0}^{p_{1}-p_{2}-1} \gamma_{i}\right)\right)=\Sigma_{a_{4}} .
$$

Now, from the Proposition 4.4.2(ii)(iv) for $\gamma_{\delta} \subset \Gamma_{a}$ (i.e., $\delta=0, \ldots, p_{1}-1$ ) there exists simple $\operatorname{arcs} \gamma_{i}$ such that $\delta=\left(i+p_{2}\right) \bmod \left(p_{1}+p_{2}\right)$ and

$$
\begin{aligned}
& f\left(\gamma_{i} \cap \Sigma_{a_{4}}\right)=\gamma_{\delta} \cap \Sigma_{a_{4}}, \quad i=0, \ldots, p_{1}-p_{2}-1 ; \\
& f\left(\gamma_{i} \cap \Sigma_{b_{2}}\right)=\gamma_{\delta} \cap \Sigma_{a_{4}}, \quad i=p_{1}, \ldots, p_{1}+p_{2}-1 ;
\end{aligned}
$$

It follows from Proposition 4.2.5, $\Sigma_{a_{4}} \subset\left[a^{r i}\left(f\left(l_{3}^{a_{4}}\right)\right) \cap \boldsymbol{a}_{\mathbf{4}}\right]^{\circ}$. On the other hand, from Equations (4.10)(4.11) for $\delta$ fixed

$$
\begin{aligned}
& f\left(\gamma_{i} \cap\left(\boldsymbol{a}_{\mathbf{4}} \cup \boldsymbol{a}_{\mathbf{5}}\right)\right) \cup f\left(\gamma_{\delta} \cap \boldsymbol{a}_{\mathbf{3}}\right)=f\left(\gamma_{i} \cap \boldsymbol{a}_{\mathbf{4}}\right) \cup f\left(\gamma_{i} \cap \boldsymbol{a}_{\mathbf{5}}\right) \cup f\left(\gamma_{\delta} \cap \boldsymbol{a}_{\mathbf{3}}\right), \quad 0 \leq i \leq p_{1}-p_{2}-1 ; \\
& \text { or } \\
& f\left(\gamma_{i} \cap\left(\boldsymbol{b}_{\mathbf{2}} \cup \boldsymbol{b}_{\mathbf{3}}\right)\right) \cup f\left(\gamma_{\delta} \cap \boldsymbol{a}_{\mathbf{3}}\right) \quad=\quad f\left(\gamma_{i} \cap \boldsymbol{b}_{\mathbf{2}}\right) \cup f\left(\gamma_{i} \cap \boldsymbol{b}_{\mathbf{3}}\right) \cup f\left(\gamma_{\delta} \cap \boldsymbol{a}_{\mathbf{3}}\right), \quad p_{1} \leq i \leq p_{1}+p_{2}-1 .
\end{aligned}
$$

Let $R=\boldsymbol{a}_{3} \cap \boldsymbol{a}_{4}$. Since the pieces

$$
\left(\left\{f\left(\gamma_{i} \cap \boldsymbol{b}_{\mathbf{3}}\right) \cup f\left(\gamma_{\delta} \cap \boldsymbol{a}_{\mathbf{3}}\right)\right\}_{i=p_{1}}^{p_{1}+p_{2}-1} \bigcup\left\{f\left(\gamma_{i} \cap \boldsymbol{a}_{\mathbf{5}}\right) \cup f\left(\gamma_{\delta} \cap \boldsymbol{a}_{\mathbf{3}}\right)\right\}_{i=0}^{p_{1}-p_{2}-1}\right) \cap R^{l e}\left(f\left(l_{3}^{a_{4}}\right)\right)
$$


not contain orbit points, up to isotopy we can deform the previous arcs, as follows (see figure 4.24(a)): First consider for $\varepsilon>0$ large enough the disk $\mathbb{D}_{\varepsilon}$ with topological diameter in $l_{4}^{a_{3}}$, as before by 2.5.2 there exist a isotopy of the identity with $\partial \mathbb{D}_{\varepsilon}$ pointwise fixed, which allows to move the pieces above inside of $R$. After this, in view of Theorem 2.5.2 again there exist a identity isotopy in some large rectangle $\Delta$, so that $R^{l e}\left(f\left(l_{3}^{a_{4}}\right)\right) \subset \Delta^{\circ}$ and the isotopy pushes one by one the arcs $f\left(\gamma_{i} \cup \gamma_{\delta}\right)$ outside of $R^{l e}\left(f\left(l_{3}^{a_{4}}\right)\right)$ passing through $l_{2}^{a_{4}}$ (see figure $\left.4.24(\mathrm{~b})\right)$. We note that $R^{r i}\left(f\left(l_{3}^{a_{4}}\right)\right)$ is a closed rectangular region and the pieces $f\left(\gamma_{i} \cap \boldsymbol{a}_{\mathbf{4}}\right) \cup f\left(\gamma_{i} \cap \boldsymbol{b}_{\mathbf{2}}\right)$ are cross-cuts in this region with endpoints $l_{4}^{a_{3}}$ and $l_{2}^{a_{4}}$, for which

$$
f\left(\gamma_{p_{1}} \cap \boldsymbol{b}_{\mathbf{2}}\right) \prec \cdots \prec f\left(\gamma_{p_{1}+p_{2}-1} \cap \boldsymbol{b}_{\mathbf{2}}\right) \prec f\left(\gamma_{0} \cap \boldsymbol{a}_{\mathbf{4}}\right) \prec \cdots \prec f\left(\gamma_{p_{1}-p_{2}-1} \cap \boldsymbol{a}_{\mathbf{4}}\right) .
$$

If we denote these pieces (i.e., $\left.\left\{f\left(\gamma_{i} \cap \boldsymbol{a}_{\mathbf{4}}\right) \cup f\left(\gamma_{i} \cap \boldsymbol{b}_{\mathbf{2}}\right)\right\} \cap R^{r i}\left(f\left(l_{3}^{a_{4}}\right)\right)\right)$ by

$$
f\left(\gamma_{p_{1}}\right) \prec \cdots \prec f\left(\gamma_{p_{1}+p_{2}-1}\right) \prec f\left(\gamma_{0}\right) \prec \cdots \prec f\left(\gamma_{p_{1}-p_{2}-1}\right),
$$

from (4.13) we have for $i \in\left\{p_{1}, \ldots, p_{1}+p_{2}-1\right\} \cup\left\{0, \ldots, p_{1}-p_{2}-1\right\}$ and $\delta=\left(i+p_{2}\right) \bmod \left(p_{1}+p_{2}\right)$ that

$$
\bigcup_{i}\left(f\left(\gamma_{i}\right) \cap \gamma_{\delta}\right) \supset \Sigma_{a_{4}}
$$

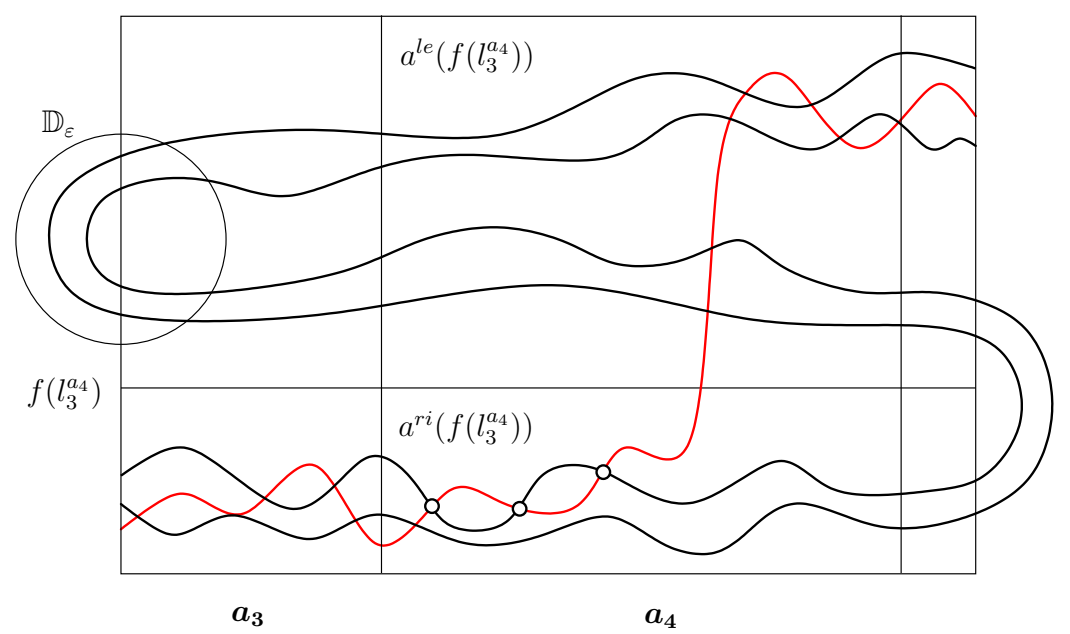

(a)

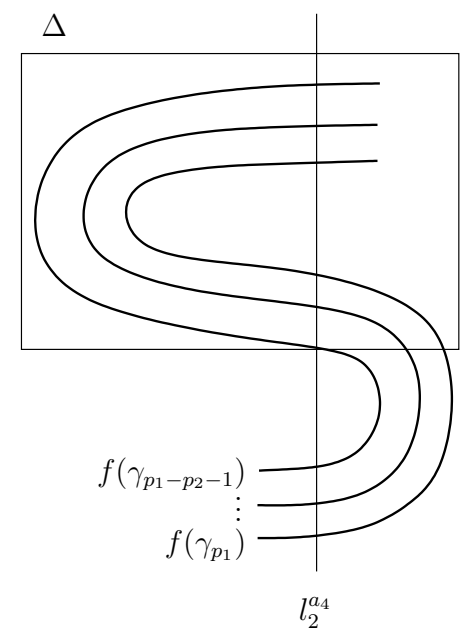

$(b)$

Figure 4.24: Isotopic deformation of the arcs $f\left(\left(\gamma_{i} \cap\left(\boldsymbol{a}_{\mathbf{5}}\right) \cup\left(\gamma_{\delta} \cap \boldsymbol{a}_{\mathbf{3}}\right)\right) ; i \in\left\{p_{1}, \ldots, p_{1}+p_{2}-1\right\} \cup\left\{0, \ldots, p_{1}-\right.\right.$ $\left.p_{2}-1\right\}$ and $\delta=\left(i+p_{2}\right) \bmod \left(p_{1}+p_{2}\right)$.

Henceforth, we will describe the isotopy in $R^{r i}\left(f\left(l_{3}^{a_{4}}\right)\right)$. By Theorem 2.5.6 for

$$
\mathbb{D}_{\delta}^{\circ}=\left[R^{l e}\left(\gamma_{\delta}\right)\right]^{\circ} \cap\left[R^{l e} f\left(\gamma_{i}\right)\right]^{\circ} \text {, and } \mathbb{D}_{\sigma}^{\circ}=\left[R^{r i}\left(\gamma_{\delta}\right)\right]^{\circ} \cap\left[R^{r i} f\left(\gamma_{i}\right)\right]^{\circ} ;
$$

the sets $\mathbb{D}_{\delta}=\overline{\mathbb{D}}_{\delta}^{\circ}$ and $\mathbb{D}_{\sigma}=\overline{\mathbb{D}}_{\sigma}^{\circ}$ are closed topological disk (see figure 4.25). Let $I=\overline{\partial \mathbb{D}_{\delta} \backslash \partial R}$ and $J=\overline{\partial \mathbb{D}_{\sigma} \backslash \partial R}$. Notice that the arcs $f\left(\gamma_{i}\right), \gamma_{\delta}$ are contained in $R^{r i}(I)$ and $R^{l e}(J)$, and moreover $f\left(\gamma_{i-1}\right) \prec f\left(\gamma_{i}\right) \prec f\left(\gamma_{i+1}\right)$. Here $f\left(\gamma_{i+1}\right)$ and $f\left(\gamma_{i-1}\right)$ may or may not intersect $I$ and $J$ respectively. In particular, if $f\left(\gamma_{i+1}\right)$ intersect $I$ (similarly $f\left(\gamma_{i-1}\right)$ and $J$ ) we can assume that they intersect transversally (i.e., the intersection define bigons between them), otherwise the isotopy is clear. Since $I$ is form by pieces of $f\left(\gamma_{i}\right)$ and $\gamma_{\delta}, f\left(\gamma_{i+1}\right)$ must intersect only the $\gamma_{\delta}$ pieces. Then, up to isotopy relative to $\Sigma_{a_{4}}$, we can remove the transversal intersections between them, pushing up $f\left(\gamma_{i+1}\right)$ to the interior of the disk $\mathbb{D}_{\delta}^{\circ}$. If many arcs intersect $I$ (with index greater or equal than $i+1$ ), we remove them one by one starting with the arc of greater index (see figure 4.25). 


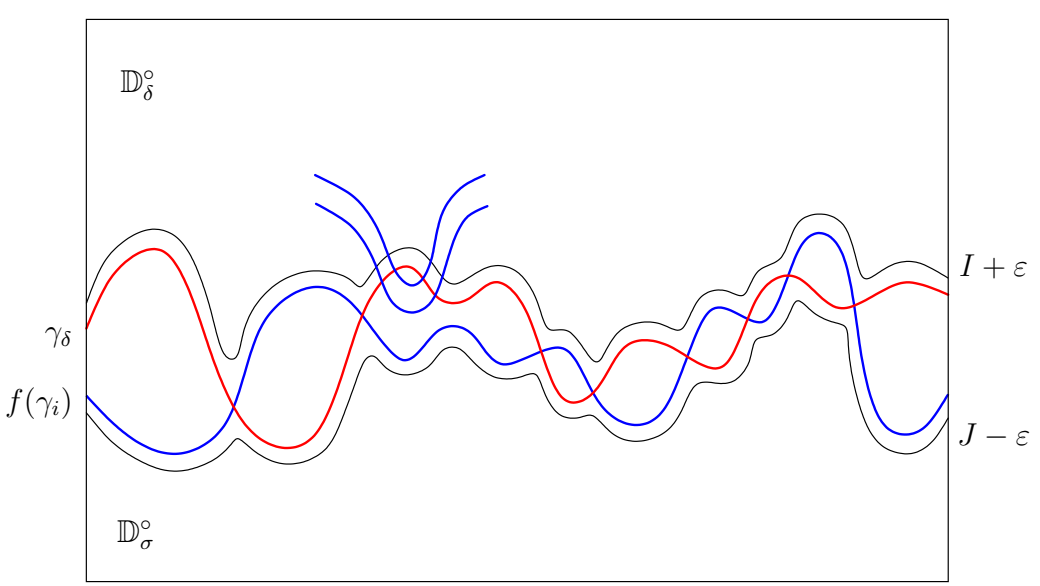

Figure 4.25: $f\left(\gamma_{i} \cap \boldsymbol{a}_{\mathbf{4}}\right) \simeq \gamma_{\delta} \cap \boldsymbol{a}_{\mathbf{4}}\left(\right.$ rel. $\left.\left\{a_{4}^{(\delta, 1)}, \ldots, a_{4}^{(\delta, k)}\right\}\right) ; i \in\left\{p_{1}, \ldots, p_{1}+p_{2}-1\right\} \cup\left\{0, \ldots, p_{1}-p_{2}-1\right\}$ and $\delta=\left(i+p_{2}\right) \bmod \left(p_{1}+p_{2}\right)$.

More clearly, the isotopy occurs as follows: For $\varepsilon>0$ small enough we define the simple arcs $I+\varepsilon, J-\varepsilon$ such that $(I+\varepsilon)^{\circ} \subset \mathbb{D}_{\delta}^{\circ}$ and $(J-\varepsilon)^{\circ} \subset \mathbb{D}_{\sigma}^{\circ}$ are at a distance $\varepsilon$ from $I$ and $J$ respectively. By Corollary 2.5.5 the bounded component by $I+\varepsilon, J-\varepsilon$ is a closed topological disk $\mathbb{D}\left(f\left(\gamma_{i}\right), \gamma_{\delta}\right)$. By construction $\left[f\left(\gamma_{i}\right) \cup \gamma_{\delta}\right]^{\circ} \subset \mathbb{D}^{\circ}$ except by the endpoints in $\partial R$. In $\boldsymbol{a} \backslash R^{\circ}$ the disjoint collection of $\operatorname{arcs}\left\{f\left(\gamma_{i}\right)\right\}$ and $\left\{\gamma_{\delta}\right\}$ have not orbit points, thus it is not hard to show that each arc $f\left(\gamma_{i}\right)$ is isotopic to $\gamma_{\delta}$. Then, we can assume the endpoints of these pairwise arcs are the same in $\partial R$.

By hypothesis the arcs $\bigcup f\left(\gamma_{i}\right), \bigcup \gamma_{\delta}$ are $a$-monotones in $R$. Starting the relative isotopy for the arcs with index $i=p_{1}$ and $\delta=0$, it follows from Corollary 2.5.2 that between each continuous pair of intersection points in $f\left(\gamma_{i}\right) \cap \gamma_{\delta}$ there exist a isotopy of the identity into $\left[\mathbb{D}\left(f\left(\gamma_{p_{1}}\right), \gamma_{0}\right)\right]^{\circ}$ that fixed the endpoints pointwise (we suppose that there are only finitely many intersection points). We continue with the method until the pairwise arcs with index $i=p_{1}-p_{2}-1$ and $\delta=p_{1}-1$.

Case $\left(\ell_{a}=\ell_{a}^{p_{1}-1}>0\right)$. By hypothesis the $\operatorname{arcs} \gamma_{\delta}\left(\delta=0, \ldots, p_{1}-2\right)$ have not $a_{3}$ 's points. From Part I (4.12), the pieces $f\left(\gamma_{i} \cup \gamma_{\delta}\right)$ intersect $\boldsymbol{a} \cap \mathbb{V}$ but these can be carried inside of $\boldsymbol{a}$ (as before by a disks $\mathbb{D} \varepsilon$ that intersect $\left.l_{2}^{a_{5}}\right)$ such that

$$
f\left(\gamma_{p_{1}} \cup \gamma_{0}\right) \prec \cdots \prec f\left(\gamma_{p_{1}+p_{2}-1} \cup \gamma_{p_{2}-1}\right) \prec f\left(\gamma_{0} \cup \gamma_{p_{2}}\right) \prec \cdots \prec f\left(\gamma_{p_{1}-p_{2}-1} \cup \gamma_{p_{1}-1}\right) .
$$

Thus,

$$
\bigcup_{\delta=0}^{p_{1}-2} f\left(\gamma_{i} \cup \gamma_{\delta}\right) \subset a^{r i}\left(f\left(\gamma_{p_{1}-p_{2}-1} \cup \gamma_{p_{1}-1}\right)\right) .
$$

These arcs are in similar conditions to the previous case, so we will assume that them can be deform by isotopy up to $a^{r i}\left(f\left(l_{3}^{a_{4}}\right)\right)$ as in 4.14. Done this, by analogy with the first case suppose that the $\operatorname{arcs} \gamma_{\delta}$ are deform isotopically to $f\left(\gamma_{i}\right)$ relative to $\Sigma_{a_{4}}$ with support in $\boldsymbol{a}$ (in the first case the support is given in $R=\boldsymbol{a}_{\mathbf{3}} \cup \boldsymbol{a}_{\mathbf{4}}$ ).

Otherwise, by (4.11)(4.12)

$$
f\left(\gamma_{p_{1}-p_{2}-1} \cap\left(\boldsymbol{a}_{\mathbf{4}} \cup \boldsymbol{a}_{\mathbf{5}}\right)\right) \cup f\left(\gamma_{p_{1}-1} \cap \boldsymbol{a}_{\mathbf{3}}\right):=f\left(\gamma_{p_{1}-p_{2}-1} \cup \gamma_{\delta}\right) .
$$

where by Propositions 4.4.1(ii), 4.4.2(iii)(iv)

$$
f\left(\gamma_{p_{1}-p_{2}-1} \cap \Sigma_{a_{4}}\right) \cup f\left(\gamma_{p_{1}-1} \cap \Sigma_{a_{3}}\right)=\gamma_{p_{1}-1} \cap\left(\Sigma_{a_{3}} \cup \Sigma_{a_{4}}\right) .
$$

We have that $f\left(\gamma_{p_{1}-p_{2}-2}\right)=\gamma_{p_{1}-2}$. Since $f\left(\gamma_{p_{1}-p_{2}-1}\right), \gamma_{p_{1}-1}$ are $a$-monotones arcs and

$$
\left[f\left(\gamma_{p_{1}-p_{2}-1}\right) \cup \gamma_{p_{1}-1}\right]^{\circ} \subset\left[a^{l e}\left(\gamma_{p_{1}-2}\right)\right]^{\circ},
$$


by Corollary 2.5.2 it follows the isotopy (see figure 4.26).

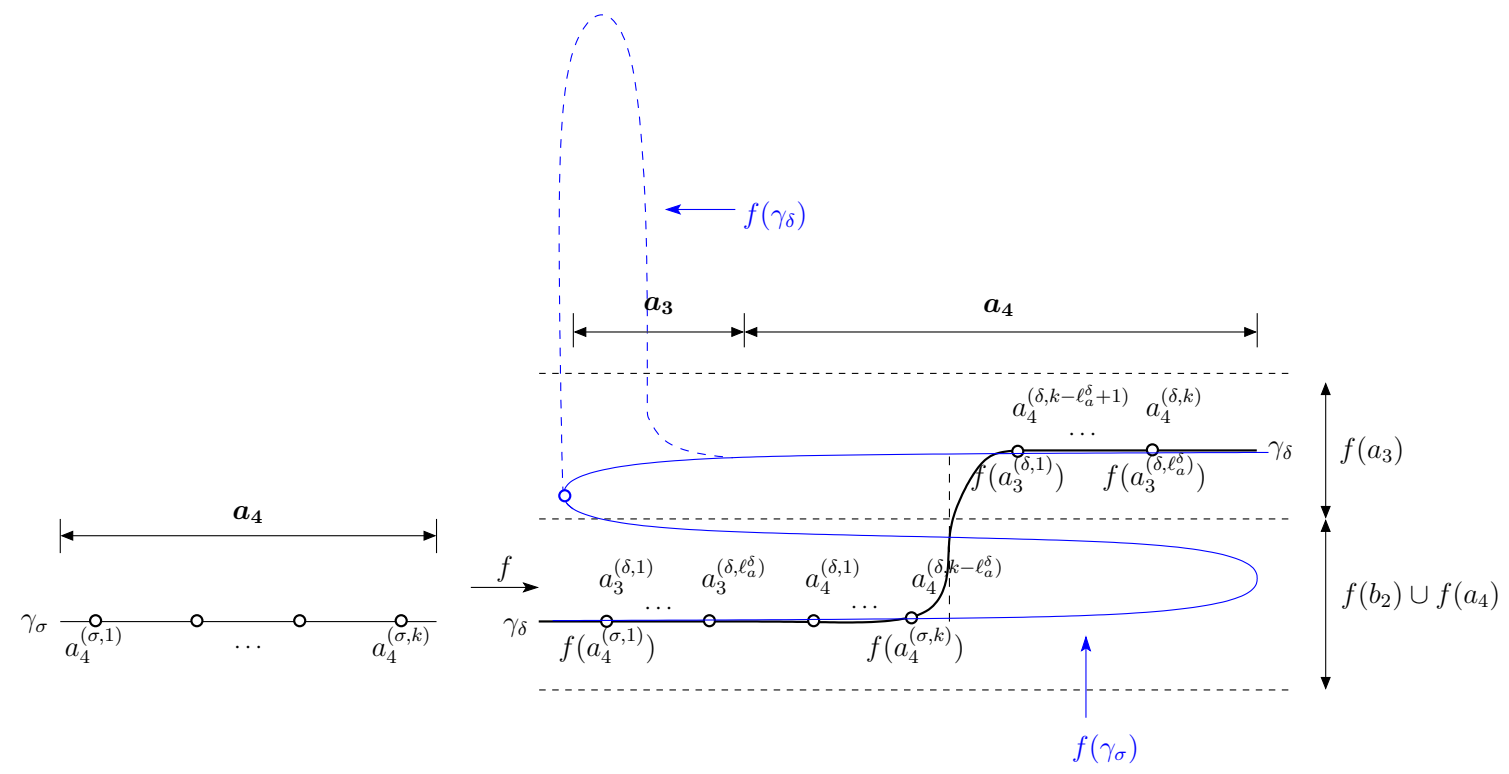

Figure 4.26: Representation of the relative isotopy for the case $\ell_{a}=\ell_{a}^{p_{1}-1}$, where $0<\ell_{a}^{\delta=p_{1}-1} \leq k$ and $\sigma=p_{1}-p_{2}-1$

Case $\left(\ell_{a}^{j}>0,0 \leq j \leq p_{1}-2\right)$. Assume that $\gamma_{j}$ is the first $a$-arc with $a_{3}$ 's points with $0 \leq i \leq p_{1}-2$. For $0 \leq j \leq i-1$, we have $\ell_{a}^{j}=0$. Thus, for $\delta=0, \ldots, j-1$ we see that $\ell_{a}^{\delta}=0$. So, using arguments similar to those shown in the first part of the previous cases, the $\operatorname{arcs} \cup_{\delta=0}^{j-1}$ can be deformed by isotopy up to them are contained in $a^{r i}\left(f\left(l_{3}^{a_{4}}\right)\right)$. By propositions 4.4.1(ii), 4.4.2(ii)(iii)(iv), for $\delta=\left(i+p_{2}\right) \bmod \left(p_{1}+p_{2}\right)$

$$
\begin{aligned}
& f\left(\gamma_{i} \cap \Sigma_{b_{2}}\right) \cup f\left(\gamma_{i} \cap \Sigma_{a_{3}}\right)=\gamma_{\delta} \cap\left(\Sigma_{a_{3}} \cup \Sigma_{a_{4}}\right), \quad i=p_{1}, \ldots, p_{1}+p_{2}-1 ; \\
& f\left(\gamma_{i} \cap \Sigma_{a_{4}}\right) \cup f\left(\gamma_{i} \cap \Sigma_{a_{3}}\right)=\gamma_{\delta} \cap\left(\Sigma_{a_{3}} \cup \Sigma_{a_{4}}\right), \quad i=0, \ldots, p_{1}-p_{2}-1 .
\end{aligned}
$$

It follows from Equation (4.11)

$$
\begin{array}{cc}
f\left(\gamma_{i} \cap\left(\boldsymbol{b}_{\mathbf{2}} \cup \boldsymbol{b}_{\mathbf{3}}\right)\right) \cup f\left(\gamma_{\delta} \cap \boldsymbol{a}_{\mathbf{3}}\right) & i=p_{1}, \ldots, p_{1}+p_{2}-1 ; \\
f\left(\gamma_{i} \cap\left(\boldsymbol{a}_{\mathbf{4}} \cup \boldsymbol{b}_{\mathbf{3}}\right)\right) \cup f\left(\gamma_{\delta} \cap \boldsymbol{a}_{\mathbf{3}}\right) & i=0, \ldots, p_{1}-p_{2}-1 .
\end{array}
$$

Let $R=\boldsymbol{a}_{\mathbf{3}} \cup \boldsymbol{a}_{\mathbf{4}}$. From the hypothesis the $\Gamma_{a}$ are $a$-monotones. If $\Gamma=\gamma_{\delta_{0}} \cup \cdots \gamma_{\delta_{n}}$ represent the arcs with $a_{3}$ 's for $j=\delta_{0}<\delta_{1}<\cdots<\delta_{n} \leq p_{1}-1$ (i.e., $\Gamma \subseteq \Gamma_{a}$ ), as we saw in the third case Theorem 4.4.3 the $\operatorname{arcs} \gamma_{\delta}$ with $\delta_{m}<\delta<\delta_{m+1}$ (i.e., without $a_{3}$ 's points) can be extended up to the leafs $E_{\delta_{m}}, G_{\delta_{m}}, m=1, \ldots, n$. Without loss of generality, we may assume that in the closed rectangular region $R^{*}$ between $f\left(l_{1}^{a_{3}}\right), f\left(l_{3}^{a_{4}}\right)$ ( $R^{*}$ is well define by 2.5.5) each simple arc $\gamma_{\delta} \cap R^{*}$ only consist of a monotonous geodesic piece (ver figure 4.27 ). 


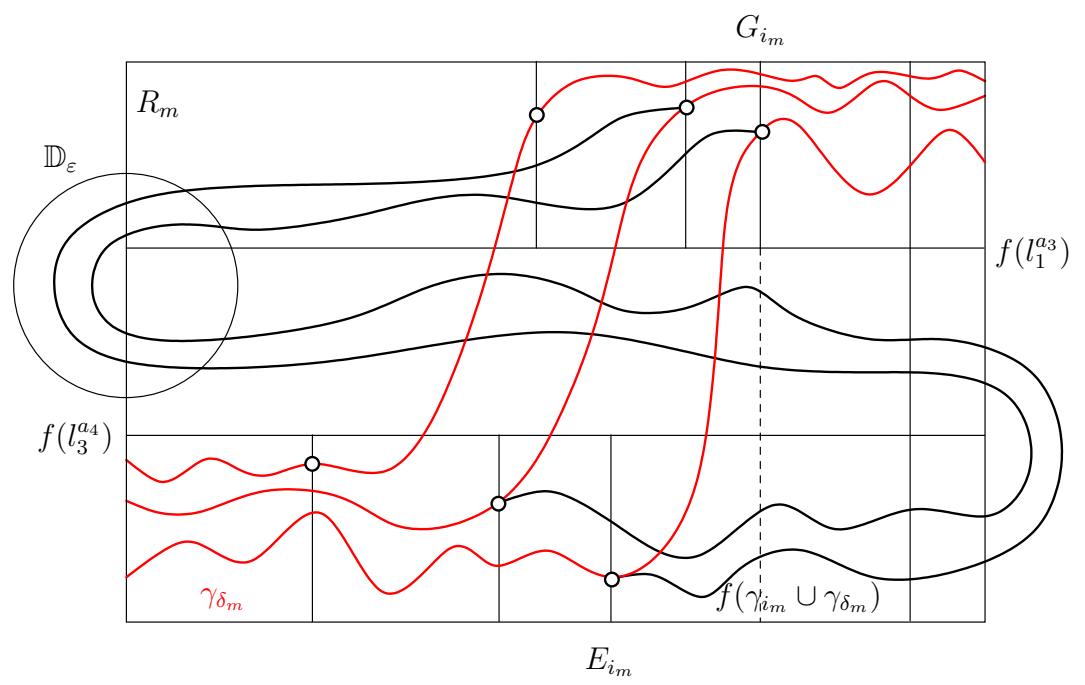

Figure 4.27: Let $f\left(\gamma_{i_{m}} \cup \gamma_{\delta_{m}}\right) \simeq \gamma_{\delta_{m}}\left(\right.$ rel. $\left.\left\{E_{i_{m}}, G_{i_{m}}\right\}\right) ; m=0, \ldots, n$ with $0 \leq \delta_{i_{m}}<\delta_{i_{m+1}}<\cdots<\delta_{i_{n}} \leq$ $p_{1}-1$.

By corollaries (4.4.6)(4.4.7)(ii) the orbit points set contained in $\left[f\left(\boldsymbol{b}_{\mathbf{2}}\right) \cup f\left(\boldsymbol{a}_{\mathbf{4}}\right)\right]^{\circ}$ are bounded by the leafs $l_{4}^{a_{3}}, E_{\delta_{0}}$ and in $\left[f\left(\boldsymbol{a}_{3}\right)\right]^{\circ}$ by $G_{\delta_{n}}, l_{2}^{a_{4}}$. By proposition $4.2 .5, R^{l e}\left(f\left(l_{1}^{a_{3}}\right)\right) \supset f\left(\boldsymbol{a}_{\mathbf{3}}\right) \cap R$ and $R^{r i}\left(f\left(l_{3}^{a_{4}}\right)\right) \supset f\left(\boldsymbol{b}_{\mathbf{2}} \cup \boldsymbol{a}_{\mathbf{4}}\right) \cap R$ are closed rectangular regions. Thus following the development of the relative isotopy in the proof of the first case, we can do isotopy between $f\left(\gamma_{i} \cup \gamma_{\delta}\right)$ and $\gamma_{\delta}$, relative to the sets $\mathcal{O} \cap f\left(\boldsymbol{a}_{\mathbf{3}}\right), \mathcal{O} \cap f\left(\boldsymbol{b}_{\mathbf{2}} \cup \boldsymbol{a}_{\mathbf{4}}\right)$ with support in $R^{l e}\left(f\left(l_{1}^{a_{3}}\right)\right)$ and $R^{r i}\left(f\left(l_{3}^{a_{4}}\right)\right)$ respectively.

In order to see the relative isotopy we start by taking two disk $\mathbb{D}_{\varepsilon}, \mathbb{D}_{\varepsilon^{\prime}}$, such that $\mathbb{D}_{\varepsilon} \cap l_{4}^{a_{3}}$, $\mathbb{D}_{\varepsilon^{\prime}} \cap l_{2}^{a_{4}}$ are topological diameters on their respective disk. From 4.12 assume that the two pieces of the $\operatorname{arcs} f\left(\gamma_{i} \cup \gamma_{\delta}\right)$ outside of $R$ are contained in $\mathbb{D}_{\varepsilon}$ and $\mathbb{D}_{\varepsilon^{\prime}}$. By Corollary 2.5.2 up to isotopy we may push these pieces inside of $R$. Now, consider the $\operatorname{arcs} \gamma_{\delta}\left(\delta_{m}<\delta<\delta_{m+1}\right)$ which have not $a_{3}$ 's points (if there exists one). By Proposition 4.4.7(i), $E_{\delta_{m}}<G_{\delta_{m}} \leq F_{\delta_{m}}$ for $m=0, \ldots, n$.

Let

$$
P_{m}=\left\{f\left(\gamma_{i} \cup \gamma_{\delta}\right) \cap G_{\delta_{m}} \mid \delta_{m}<\delta<\delta_{m+1}\right\} .
$$

Since the pieces $f\left(\gamma_{i}\right), f\left(\gamma_{\delta}\right)$ are $a$-monotones (ever after isotopic deformation in the disk $\mathbb{D}_{\varepsilon}$, $\left.\mathbb{D}_{\varepsilon^{\prime}}\right)$, there are exactly two points for each transverse intersection $f\left(\gamma_{i}\right) \cap G_{\delta_{m}}, f\left(\gamma_{\delta}\right) \cap G_{\delta_{m}}$. We note that the pieces $\left\{f\left(\gamma_{i} \cup \gamma_{\delta}\right) \mid\left(\delta_{m}<\delta<\delta_{m+1}\right)\right\}$ is a collection of cross-cuts in the rectangular regions

$$
R^{l e}\left(f\left(l_{3}^{a_{4}}\right)\right) \cap R^{l e}\left(G_{i_{m}}\right)=R_{m}
$$

with endpoints in $G_{\delta_{m}}$ (see figure 4.27 ). Notice that $R_{m}$ contains a piece of each $\gamma_{\delta}\left(F_{\delta_{m}}\right)<G_{\delta_{m}}$ in which we will deform to $f\left(\gamma_{i} \cup \gamma_{\delta}\right)$ by isotopy. In view of Corollary 2.5.2 we can do isotopy relative to $P_{m} \cap f\left(\boldsymbol{a}_{\mathbf{3}}\right)$ and

$$
\begin{aligned}
& Q_{m}=\left\{\gamma_{\delta} \cap f\left(\gamma_{i} \cup \gamma_{\delta}\right) \mid \delta_{m}<\delta<\delta_{m+1}\right\} \bigcap R^{*} ; \\
& =\left\{\gamma_{\delta} \cap f\left(\gamma_{i}\right) \mid \delta_{m}<\delta<\delta_{m+1}\right\} .
\end{aligned}
$$

More precisely,

$$
f\left(\gamma_{i} \cup \gamma_{\delta}\right) \simeq \gamma_{\delta}\left(\operatorname{rel} .\left\{G_{\delta_{m}} \cap \gamma_{\delta}, f\left(\gamma_{i}\right) \cap \gamma_{\delta}\right\}\right)
$$

with support in $R_{m}$, where $\delta=\left(i+p_{2}\right) \bmod \left(p_{1}+p_{2}\right)$. The isotopy should begin by the arc with lowest index (i.e., $\delta=\delta_{0}$ ) deforming the arcs one by one as the index increases. The rest of the isotopy follows in the same way on regions $R^{r i}\left(f\left(l_{1}^{a_{3}}\right)\right) \cap R^{l e}\left(E_{\delta_{m}}\right)$ starting by the arcs with greater index (i.e., $\delta=p_{1}-1$ ). Since Since on the region a1 it is enough to repeat the previous method, this completes the isotopy on $a$-strip. 
Once the isotopy between $f(\gamma)$ and $\gamma$ relative to $\mathcal{O}$ has been proved for any of the previous cases, from Theorem 3.3.2, it follows that the orbit $\mathcal{O}$ is of finite order periodic orbit as we wanted.

Example 5. In particular, the pair $(\mathcal{O}, \gamma)$ of the first example presented in 3 is unfoldable. Doing isotopy on the image of the simple closed curve relative to the periodic orbit, we can see the invariance of curve. This invariance up isotopy relative is enough to show that the orbit is a finite order periodic orbit.

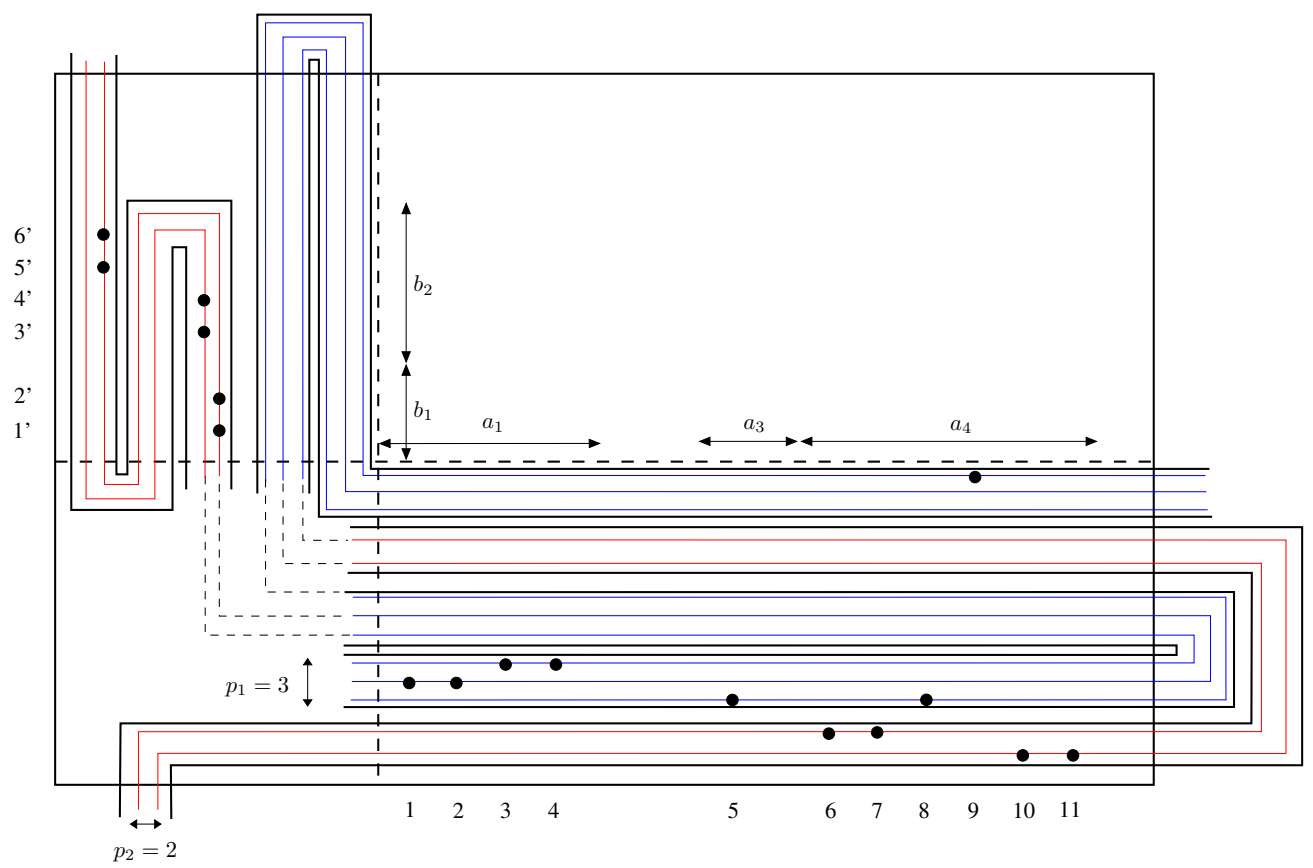

Figure 4.28: Image under $f$ of the pair $(\mathcal{O}, \gamma)$ with word $w=\left(a_{1} b_{1} b_{2} a_{4} a_{3} a_{4} a_{1} b_{2} a_{4} a_{1} b_{1} b_{2} a_{4}^{2} a_{1} b_{2} a_{4}\right)^{\infty}$. The $f(\gamma)$ curve can be deformed isotopically to $\gamma$ relative to $\mathcal{O}$.

Finally through an example, we will represent finite order periodic orbits in terms of their "inclination", that is, keeping the homological direction $\left(p_{1}, p_{2}\right)$. Using the property $4.3 .1(\mathrm{G} 2)$, we will show an example that systematically lists for each $n \geqslant k \cdot q$, a finite order periodic orbit. Note in the Table 4.3, that the symbolic description of the fopos with $\left(p_{1}, p_{2}\right)$ fixed are related with the symbolic sequence of the rational rotation vector $\frac{1}{q}\left(p_{1}, p_{2}\right)$ in the hypotenuse of the rotation triangle.

Example 6. Consider $v=\frac{k}{n}(2,1)$, the following table contains a finite order periodic orbit for each $n \geqslant k \cdot q$ : 
Table 4.3: Symbolic description of the finite order periodic orbits with rotation vector in the rotation triangle intersected with the straight line that pass through the points $(0,0),\left(\frac{2}{4}, \frac{1}{4}\right)$. On hypotenuse of rotation triangle, we first describe symbolically the orbit with rotation vector $\left(\frac{2}{4}, \frac{1}{4}\right)$, as $w=\left(b_{2} \cdot a_{4} \cdot a_{4} \cdot a_{1}\right)^{\infty}$.

\begin{tabular}{|l|l|l|}
\hline$k$ & Rotation vector & Symbolic sequence $(\cdot)^{\infty}$ \\
\hline 1 & $\left(\frac{2}{5}, \frac{1}{5}\right)$ & $b_{2} \cdot a_{4} \cdot a_{4} \cdot a_{1} \cdot b_{1}$ \\
\hline 1 & $\left(\frac{2}{6}, \frac{1}{6}\right)$ & $b_{2} \cdot a_{4} \cdot a_{4} \cdot a_{1} \cdot b_{1} \cdot b_{1}$ \\
\hline 1 & $\left(\frac{2}{7}, \frac{1}{7}\right)$ & $b_{2} \cdot a_{4} \cdot a_{4} \cdot a_{1} \cdot b_{1} \cdot b_{1} \cdot b_{1}$ \\
\hline$\vdots$ & $\vdots$ & $\vdots$ \\
\hline 2 & $\left(\frac{4}{9}, \frac{2}{9}\right)$ & $b_{2} \cdot a_{4} \cdot a_{4} \cdot a_{1} \cdot b_{1} \cdot b_{2} \cdot a_{4} \cdot a_{4} \cdot a_{1}$ \\
\hline 2 & $\left(\frac{4}{10}, \frac{2}{10}\right)=\left(\frac{2}{5}, \frac{1}{5}\right)$ & $b_{2} \cdot a_{4} \cdot a_{4} \cdot a_{1} \cdot b_{1} \cdot b_{2} \cdot a_{4} \cdot a_{4} \cdot a_{1} \cdot b_{1}$ \\
\hline 2 & $\left(\frac{4}{11}, \frac{2}{11}\right)$ & $b_{2} \cdot a_{4} \cdot a_{4} \cdot a_{1} \cdot b_{1} \cdot b_{1} \cdot b_{2} \cdot a_{4} \cdot a_{4} \cdot a_{1} \cdot b_{1}$ \\
\hline 2 & $\left(\frac{4}{12}, \frac{2}{12}\right)=\left(\frac{2}{6}, \frac{1}{6}\right)$ & $b_{2} \cdot a_{4} \cdot a_{4} \cdot a_{1} \cdot b_{1} \cdot b_{1} \cdot b_{2} \cdot a_{4} \cdot a_{4} \cdot a_{1} \cdot b_{1} \cdot b_{1}$ \\
\hline 2 & $\left(\frac{4}{13}, \frac{2}{13}\right)$ & $b_{2} \cdot a_{4} \cdot a_{4} \cdot a_{1} \cdot b_{1} \cdot b_{1} \cdot b_{1} \cdot b_{2} \cdot a_{4} \cdot a_{4} \cdot a_{1} \cdot b_{1} \cdot b_{1}$ \\
\hline$\vdots$ & $\vdots$ & $\vdots$ \\
\hline 3 & $\left(\frac{6}{13}, \frac{3}{13}\right)$ & $b_{2} \cdot a_{4} \cdot a_{4} \cdot a_{1} \cdot b_{1} \cdot b_{2} \cdot a_{4} \cdot a_{4} \cdot a_{1} \cdot b_{2} \cdot a_{4} \cdot a_{4} \cdot a_{1}$ \\
\hline 3 & $\left(\frac{6}{14}, \frac{3}{14}\right)$ & $b_{2} \cdot a_{4} \cdot a_{4} \cdot a_{1} \cdot b_{1} \cdot b_{2} \cdot a_{4} \cdot a_{4} \cdot a_{1} \cdot b_{1} \cdot b_{2} \cdot a_{4} \cdot a_{4} \cdot a_{1}$ \\
\hline 3 & $\left(\frac{6}{15}, \frac{3}{15}\right)=\left(\frac{2}{5}, \frac{1}{5}\right)$ & $b_{2} \cdot a_{4} \cdot a_{4} \cdot a_{1} \cdot b_{1} \cdot b_{2} \cdot a_{4} \cdot a_{4} \cdot a_{1} \cdot b_{1} \cdot b_{2} \cdot a_{4} \cdot a_{4} \cdot a_{1} \cdot b_{1}$ \\
\hline 3 & $\left(\frac{6}{16}, \frac{3}{16}\right)$ & $b_{2} \cdot a_{4} \cdot a_{4} \cdot a_{1} \cdot b_{1} \cdot b_{1} \cdot b_{2} \cdot a_{4} \cdot a_{4} \cdot a_{1} \cdot b_{1} \cdot b_{2} \cdot a_{4} \cdot a_{4} \cdot a_{1} \cdot b_{1}$ \\
\hline$\vdots$ & $\vdots$ & $\vdots$ \\
\hline
\end{tabular}

\subsection{Symbolic description}

Let $(\mathcal{O}, \gamma)$ be a compatible orbit curve pair with rotation vector $v=\frac{k}{n}\left(p_{1}, p_{2}\right) ; k, n$ relatively prime. From the Definition 4.2.10, $\gamma$ is a $\left(p_{1}, p_{2}\right)$ oriented simple closed curve divided by (4.3) into $p_{1}+p_{2}$ arcs, where each of the $a$-arcs $0 \leqslant i \leqslant p_{1}-1$ contain $k$ points in $\boldsymbol{a}_{\mathbf{4}}$, and each of $b$-arcs $p_{1} \leqslant i \leqslant p_{1}+p_{2}-1$ contain $2 k$ points equally distributed between $\boldsymbol{a}_{\mathbf{1}}, \boldsymbol{b}_{\mathbf{2}}$. By 4.2.10(iv) the sum of the number of points in the regions $\boldsymbol{b}_{\mathbf{1}}, \boldsymbol{a}_{\mathbf{3}}$ is constant, furthermore they can be distributed in any way between the arcs that pass through these regions. Now, we can distribute the points of the period- $n$ periodic orbit in the circular version of the $\left(p_{1}, p_{2}\right)$-curve $\gamma$ make them evenly spaced, a $2 \pi / n$ (clockwise). From $4.2 .10(\mathrm{v})$, the orbit $\mathcal{O}$ is compatible with a $2 \pi k / n$ rotation, it follows that each periodic point is pushing forward $k$ positions in the direction induce on the curve under the action of $f$. Being $k, n$ relatively prime with $k<n$, we will divide the word $w$ that represent the symbolic description in $k$-blocks. This is,

$$
w=\left(B_{1} \cdot B_{2} \cdot \ldots \cdot B_{k}\right)^{\infty}
$$

Since each of the $p_{1}+p_{2}$ arcs contains at least $k$ points in $\boldsymbol{a}_{\mathbf{1}}$, or $\boldsymbol{a}_{\mathbf{4}}$ or $\boldsymbol{b}_{\mathbf{2}}$, in each lap around $\gamma$ we have the same number of $a_{1}$ 's, $a_{4}$ 's, $b_{2}$ 's (exactly $p_{1}+2 p_{2}$ terms) and this no depend the number of $a_{3}$ 's, $b_{1}$ 's orbit points. Then, we will divide each block $B_{l}, 1 \leqslant l \leqslant k$ that represent one lap around $\gamma$, into $p_{1}$ or $p_{2}$ sub-blocks, as follows 


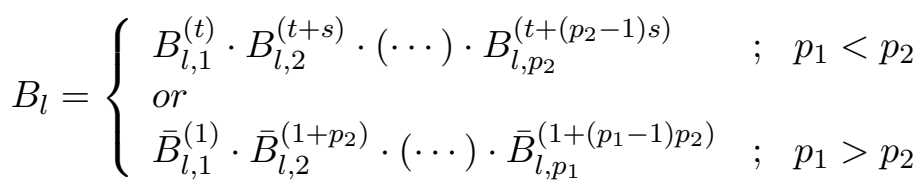

where, $t=p_{1}+1, s=p_{2}-r$ and

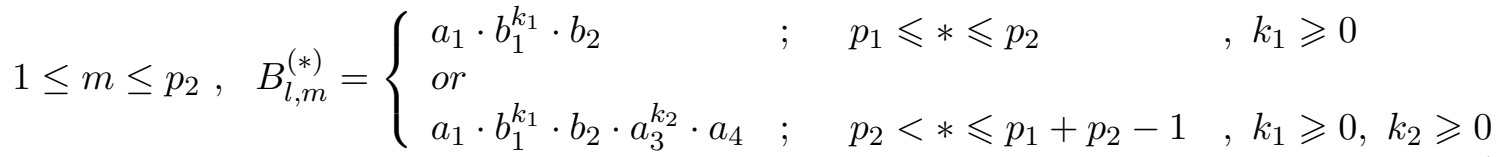

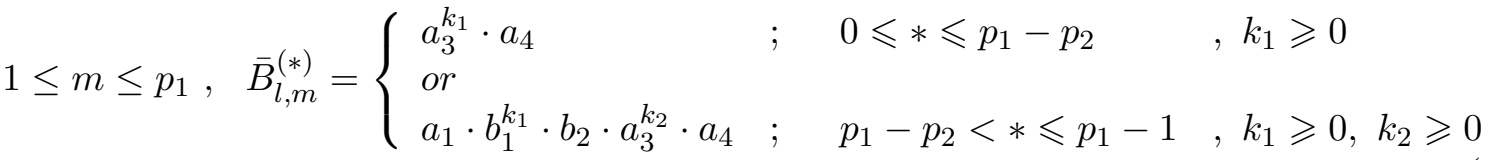

These equations follows from,

Case $\left(p_{2}>p_{1}\right)$. In this case, we divide each block $B_{l}$ in $p_{2}$ sub-blocks, which we denote as $B_{l, 1}, \ldots, B_{l, p_{2}}$. Since each block contain the same number of $a_{1}$ 's, $a_{4}$ 's, and $b_{2}$ 's orbit points in a lap in the circle version of $\gamma$, we can group them according to the $p_{2} b$-arcs. Being $p_{1}+p_{2}, p_{2}$ are relatively prime ${ }^{1}$, by the division algorithm, $p_{1}+p_{2}=m_{1} \cdot p_{2}+r_{1}$ with $1<r_{1}<p_{2}$. In the circle version of $\gamma$ the arcs $p_{1} \leqslant i \leqslant p_{1}+p_{2}-1$ satisfy the following behavior

○ To $1 \leqslant k^{\prime} \leqslant r_{1}<p_{2}$,

$$
\gamma_{\left(p_{1}+k^{\prime}\right)} \rightarrow \gamma_{\left(p_{1}+k^{\prime}\right)+p_{2}} \rightarrow \gamma_{\left(p_{1}+k^{\prime}\right)+2 p_{2}} \rightarrow \ldots \rightarrow \gamma_{\left(p_{1}+k^{\prime}\right)+m_{1} p_{2}} \rightarrow \gamma_{\left(p_{1}+k^{\prime}\right)+\left(m_{1}+1\right) p_{2}} \rightarrow
$$

Note that $\left(p_{1}+k^{\prime}\right)+m_{1} p_{2}+p_{2}=\left(p_{1}+k^{\prime}\right)+\left(p_{1}+p_{2}\right)-r_{1}+p_{2}=\left(p_{1}+k^{\prime}\right)-r_{1}+p_{2}$, then $\gamma_{\left(p_{1}+k^{\prime}\right)} \stackrel{1-l a p}{\longrightarrow} \gamma_{\left(p_{1}+k^{\prime}\right)-r_{1}+p_{2}}$.

○ To $r_{1}+1 \leqslant k^{\prime} \leqslant p_{2}$,

$$
\gamma_{\left(p_{1}+k^{\prime}\right)} \rightarrow \gamma_{\left(p_{1}+k^{\prime}\right)+p_{2}} \rightarrow \gamma_{\left(p_{1}+k^{\prime}\right)+2 p_{2}} \rightarrow \ldots \rightarrow \gamma_{\left(p_{1}+k^{\prime}\right)+m_{1} p_{2}} \rightarrow
$$

Note that $\left(p_{1}+k^{\prime}\right)+m_{1} p_{2}=\left(p_{1}+k^{\prime}\right)+\left(p_{1}+p_{2}\right)-r_{1}=\left(p_{1}+k^{\prime}\right)-r_{1}$, then we have that $\gamma_{\left(p_{1}+k^{\prime}\right)} \stackrel{1-l a p}{\longrightarrow} \gamma_{\left(p_{1}+k^{\prime}\right)-r_{1}}$.

Therefore, $\gamma_{\left(p_{1}+k^{\prime}\right)} \stackrel{1-l a p}{\longrightarrow} \gamma_{\left(\left(p_{1}+k^{\prime}\right)-r_{1}\right) \bmod \left(p_{2}\right)}$ for $1 \leqslant k^{\prime} \leqslant p_{2}$. This means that a $b$-arc on $\boldsymbol{b}$ strip come back $r_{1}$ positions to the left of this. Writing $s=p_{2}-r_{1}>0$, we have that $B_{l}, 1<l<k$ can be divided in $p_{2}$ sub-blocks $B_{l, 1}^{(*)}, B_{l, 2}^{(*+s)}, \ldots, B_{l, p_{2}}^{\left(*+\left(p_{2}-1\right) s\right)}$, where $p_{1}<*<p_{1}+p_{2}-1$. Intuitively, if we start at a point in any $b$-arc on $\boldsymbol{a}_{\mathbf{1}}$ each sub-block groups the points (from a $B_{l}$ block) of the arcs that cross $\boldsymbol{a}$ strip, before returning to another $\boldsymbol{a}_{\mathbf{1}}$ strip (which goes to $\boldsymbol{b}$ strip).

Case $\left(p_{1}>p_{2}\right)$. Similarly to the previous case, each $B_{l}$ block will be divided in $p_{1}$ sub-blocks. The sub-block $B_{l, j}, 1 \leqslant j \leqslant p_{1}$ groups the points that go to an $\gamma_{i}, 0 \leqslant i \leqslant p_{1}-1$ arc into $\boldsymbol{a}_{\mathbf{4}}$ to another arc in the same region. Doing $p_{1}+p_{2}=m_{2} p_{1}+r_{2}$ to $1<r_{2}<p_{1}$, we have

○ To $1 \leqslant k^{\prime \prime} \leqslant r_{2}<p_{1}$,

$$
\gamma_{k^{\prime \prime}} \stackrel{1-l a p}{\longrightarrow} \gamma_{k^{\prime \prime}+\left(m_{2}+1\right) p_{1}}=\gamma_{k^{\prime \prime}+\left(p_{1}+p_{2}\right)-r_{2}+p_{1}}=\gamma_{k^{\prime \prime}-r_{2}+p_{1}}
$$

\footnotetext{
${ }^{1}$ Since that $p_{1}+p_{2} \equiv p_{1} \bmod p_{2}$, we see that $\left(p_{1}+p_{2}\right) / p_{2}, p_{1} / p_{2}$ have the same rest. Now, writing $p_{1}+p_{2}=m_{1} p_{2}+r$, $p_{1}=m_{2} p_{2}+r$ by result of number theory we have $\left(p_{1}+p_{2}, p_{2}\right)=\left(p_{2}, r\right)=\left(p_{1}, p_{2}\right)=1$.
} 
○ To $r_{2}+1 \leqslant k^{\prime \prime} \leqslant p_{1}$,

$$
\gamma_{k^{\prime \prime}} \stackrel{1-l a p}{\longrightarrow} \gamma_{k^{\prime \prime}+m_{2} p_{1}}=\gamma_{k^{\prime \prime}+\left(p_{1}+p_{2}\right)-r_{2}}=\gamma_{k^{\prime \prime}-r_{2}}
$$

therefore, to $1 \leqslant k^{\prime \prime} \leqslant p_{1}$ we have $\gamma_{k^{\prime \prime}} \stackrel{1-l a p}{\longrightarrow} \gamma_{\left(k^{\prime \prime}-r_{2}\right) \bmod \left(p_{1}\right)}$. Writing $t=p_{1}-r_{2}>0$ we can divided each block $B_{l}, 1<l<k$ in $p_{1}$ sub-blocks $B_{l, 1}^{1}, B_{l, 2}^{1+t}, \ldots, B_{l, p_{1}}^{1+\left(m_{2}-1\right) t}$.

Once we symbolically describe each periodic orbit in a pair $(\mathcal{O}, \gamma)$, we can find the relative position of each orbit point whitin $\boldsymbol{a}, \boldsymbol{b}$. We finish this section by giving an example that joins the above information.

Example 7. Suppose that $v=\frac{2}{17}(3,2)$ is realized by the periodic orbit according to the Definition 4.2.10 with symbolic description

$$
w=\left(a_{1} b_{1}^{2} b_{2} a_{4}^{2} a_{1} b_{2} a_{4} a_{1} b_{1} a_{4}^{2} a_{1} b_{2} a_{4}\right)^{\infty}
$$

We know by first part of the Example 1 that there exist a curve $\gamma$ of $\operatorname{Hom}(\gamma)=(3,2)$, such that the simple arcs are connecting as, $\gamma_{1} \longrightarrow \gamma_{3} \longrightarrow \gamma_{5} \longrightarrow \gamma_{2} \longrightarrow \gamma_{4} \rightarrow$, we apply the Definition 4.2.10 for the pair $(\mathcal{O}, \gamma)$ (see the next figure). Ordering the symbolic sequence, we obtain the description to the right.

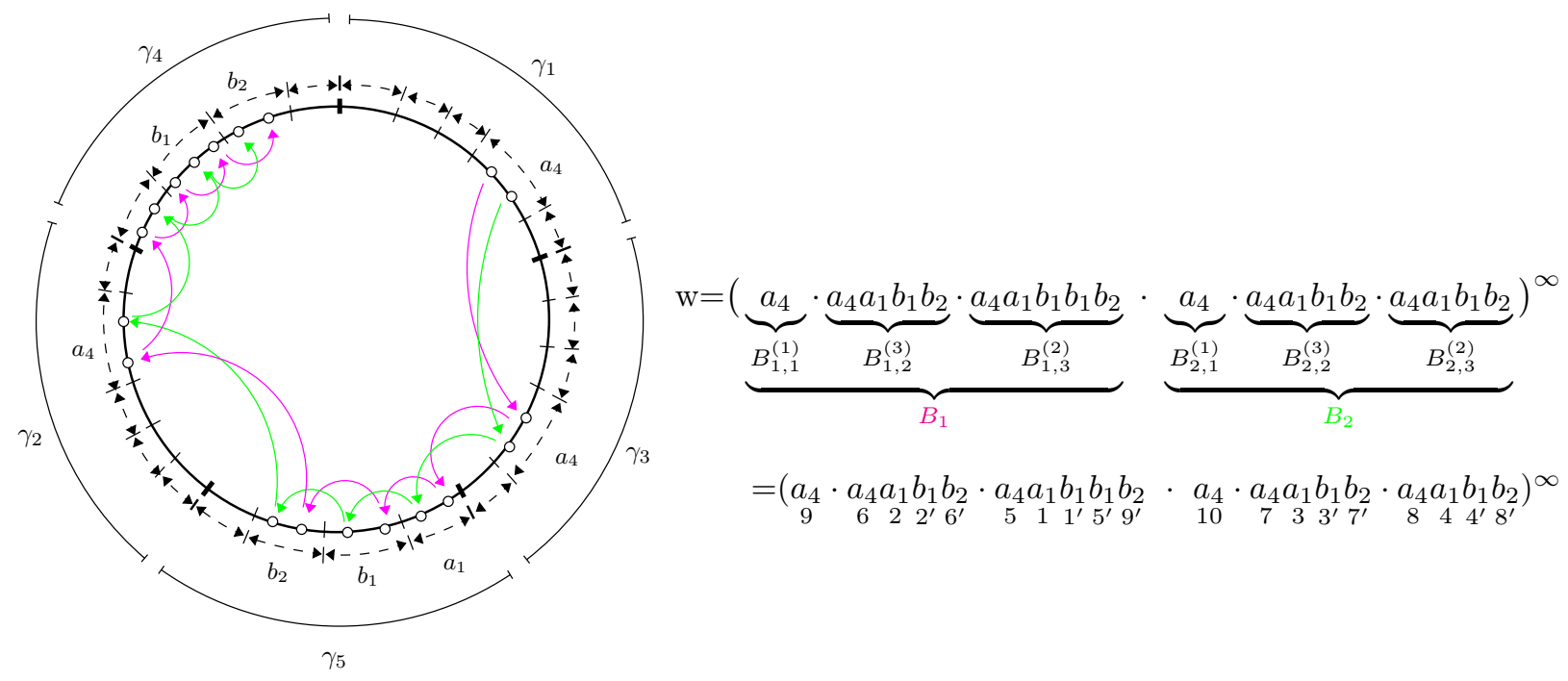

\subsection{Computational program}

Given a rational rotation vector in the two-torus is not quickly to find and list the finite order periodic orbits, for this reason we decide to implement a routine or computational program to simplified the process to find the fopos related to a rational rotation vector. The algorithm list the periodic orbits with rotation vector $v=\frac{k}{n}\left(p_{1}, p_{2}\right)$, where each one is described with a sequence symbolic which satisfy: $a_{1}:=1, a_{3}:=2, a_{4}:=3, b_{1}:=4, b_{2}:=5$. Each periodic orbit is classified as a fopo or Nofopo (Note that the Nofopo orbits are not really classified by the algorithm).

To simplify the use of the computational program, we have designed a graphical interface (See the figure below), in which you can directly enter the rotation vector, or you can randomly choose a vector by clicking on the button $\left(k, n, p_{1}, p_{2}\right)$ (for example $n$ takes some value between 1 and 50 , etc.). The computational application also create a .txt file with symbolic sequences. 


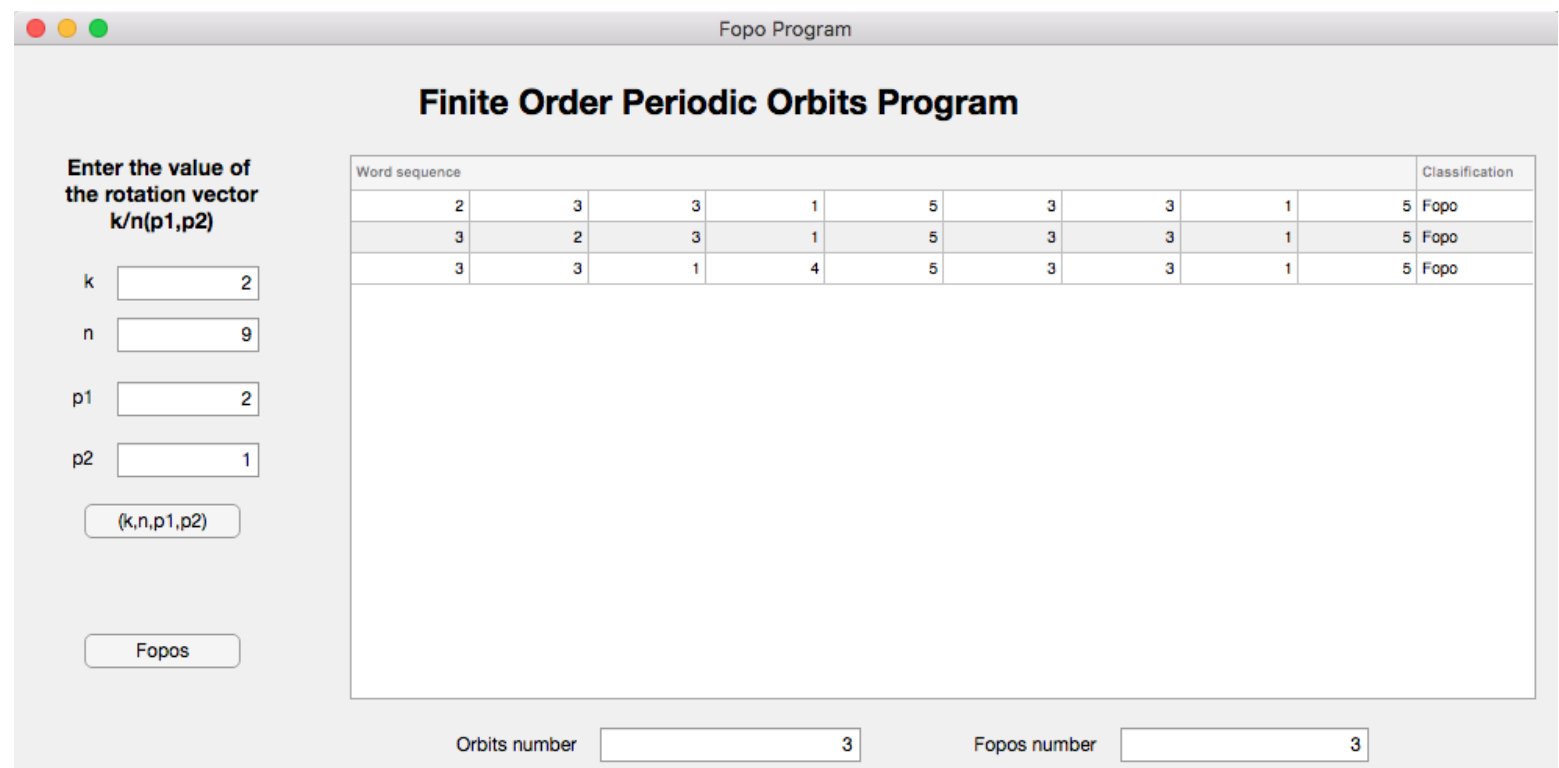

For the distribution of the application, you can get it for free by writing to the email german.escobar@usco.edu.co. We have two options:

1. We give a link to download the installers for windows or mac (500 Mb approximately).

2. In the case of having Matlab (the routine was built in this language) we share the folder that contains the code and functions that make up the application $(12 \mathrm{~Kb})$. 


\section{Bibliography}

[BdCH16] P. Boyland, A. de Carvalho e T. Hall. New rotation sets in a family of torus homeomorphisms. Invent. math., 204:895-937, 2016. First Online: 14 October 2015. 1, 9

[BH95] M. Bestvina e M. Handel. Train-tracks for surface homeomorphism. Topology, 34:109140, 1995. 4

[Boy92] P. Boyland. Rotation sets and monotone orbits for annular homeomorphisms. Comm. Math. Helv., 67:202-213, 1992. 1

[Bus10] P. Buser. Geometry and Spectra of Compact Riemann Surfaces. Birkhäuser, 2010. Reprint of the 1992 Edition. 5

[CK94] A. Constantin e B. Kolev. The theorem of kerékjártó on periodic homeomorphisms of the disc and the sphere. Enseign. Math. (2), 40(3-4):193-204, 1994. 6

[dCH01] A. de Carvalho e T. Hall. Pruning theory and thurstons classification of surface homeomorphisms. J. Eur. Math. Soc., 3:287-333, 2001. 4

[FM93] J. Franks e M. Misiurewicz. Cycles for disk homeomorphisms and thick trees. Em Nielsen theory and dynamical systems (1992, South Hadley, MA), páginas 69-139. Amer. Math. Soc., Providence, RI, 1993. 4

[FM12] B. Farb e D. Margalit. A primer on mapping class group. Princeton University Press, first edição, 2012. 5

[Fra89] J. Franks. Realizing rotation vectors for torus homeomorphisms. Trans. Am. Math. Soc., 311:107-115, 1989. 1, 4

[GLM91] J. Guaschi, J. Llibre e R. Mackay. A classification of braid types for periodic orbits of diffeomorphism of surfaces of genus one with topological entropy zero. Publ. Mat., Barc., 35:543-558, 1991. 1, 6

[Kol94] B. Kolev. Plane topology and dynamical systems. https://cel.archives-ouvertes.fr/cel00719540/file/grenoble94.pdf, 1994. 6

[Lee11] J. M. Lee. Introduction to topological manifolds. 2nd ed, volume 202. New York, NY: Springer, 2nd ed. edição, 2011. 5

[MT01] B. Mohar e C. Thomassen. Graphs on surfaces. Baltimore, MD: Johns Hopkins University Press, 2001. 5

[MZ89] M. Misiurewics e K. Ziemian. Rotation sets for maps of tori. J. London Math. Soc., 40:490-506, 1989. 4

[Par05] K. Parwani. Monotone periodic orbits for torus homeomorphisms. Proc. Amer. Math. Soc., 133:1677-1683, 2005. 1, 12, 15

[Why58] G. T. Whyburn. Topological analysis. Princeton, N. J.: Princeton University Press XII, 1958. 5 